\title{
Invariants of Fold-maps via Stable Homotopy Groups
}

\author{
Dedicated to Professor Tatsuo Suwa on his sixtieth birthday
}

By

Yoshifumi ANDO*

\begin{abstract}
In the 2-jet space $J^{2}(n, p)$ of smooth map germs $\left(\mathbf{R}^{n}, 0\right) \rightarrow\left(\mathbf{R}^{p}, 0\right)$ with $n \geq p \geq$ 2 , we consider the subspace $\Omega^{n-p+1,0}(n, p)$ consisting of all 2 -jets of regular germs and map germs with fold singularities. In this paper we determine the homotopy type of the space $\Omega^{n-p+1,0}(n, p)$. Let $N$ and $P$ be smooth $\left(C^{\infty}\right)$ manifolds of dimensions $n$ and $p$. A smooth map $f: N \rightarrow P$ is called a fold-map if $f$ has only fold singularities. We will prove that this homotopy type is very useful in finding invariants of fold-maps. For instance, by applying the homotopy principle for fold-maps in [An3] and [An4] we prove that if $n-p+1$ is odd and $P$ is connected, then there exists a surjection of the set of cobordism classes of fold-maps into $P$ to the stable homotopy group $\lim _{k, \ell \rightarrow \infty} \pi_{n+k+\ell}\left(T\left(\nu_{P}^{k}\right) \wedge T\left(\widehat{\gamma}_{G_{n-p+1, \ell}}^{\ell}\right)\right)$. Here, $\nu_{P}^{k}$ is the normal bundle of $P$ in $\mathbf{R}^{p+k}$ and $\widehat{\gamma}_{G_{n-p+1, \ell}}^{\ell}$ denote the canonical vector bundles of dimension $\ell$ over the grassman manifold $G_{n-p+1, \ell}$. We also prove the oriented version.
\end{abstract}

\section{Introduction}

Let $N$ and $P$ be smooth $\left(C^{\infty}\right)$ manifolds of dimensions $n$ and $p$ with $n \geq p \geq 2$. A fold-map germ $(N, x) \rightarrow(P, y)$ refers to a smooth map germ which is written as $\left(x_{1}, \ldots, x_{n}\right) \mapsto\left(x_{1}, \ldots, x_{p-1}, \pm x_{p}^{2} \pm \cdots \pm x_{n}^{2}\right)$ under suitable local coordinates systems of $(N, x)$ and $(P, y)$. A fold-map $N \rightarrow P$ refers to a smooth

Communicated by K. Saito. Received October 26, 2001.

2000 Mathematics Subject Classification(s): Primary 57R45; Secondary 58A20, 55Q10.

*Department of Mathematical Sciences, Yamaguchi University, Yamaguchi 753-8512, Japan.

e-mail: andoy@po.cc.yamaguchi-u.ac.jp.

This research was partially supported by Grant-in-Aid for Scientific Research (No. 11640081), Ministry of Education, Science, Sports and Culture, Japan. 
map which has only fold singularities. In this paper we will study the existence problem of fold-maps and homotopy-theoretic invariants for classifying foldmaps from the viewpoint of homotopy principle (the terminology used in [G2]).

Let $J^{2}(N, P)$ denote the 2-jet space of the manifolds $N$ and $P$ and let $\Omega^{n-p+1,0}(N, P)$ be the subspace of $J^{2}(N, P)$ associated to $\Omega^{n-p+1,0}(n, p)$, which consists of all 2 -jets of regular germs and fold-map germs. We explain the motivation for studying the homotopy type of $\Omega^{n-p+1,0}(n, p)$. The existence and non-existence problem of fold-maps has been first dealt with in dimensions $(n, 2)$ in [T] and [L]. A smooth map $f: N \rightarrow P$ is a fold-map if and only if the image of $j^{2} f$ is contained in $\Omega^{n-p+1,0}(N, P)$ and $j^{2} f$ is transverse to the Boardman submanifold $\Sigma^{n-p+1,0}(N, P)$ defined in [L] and [B] (see [Mo]). Let $C_{\Omega}^{\infty}(N, P)$ denote the space consisting of all smooth maps $f: N \rightarrow P$ such that the image of $j^{2} f$ is contained in $\Omega^{n-p+1,0}(N, P)$ with the $C^{\infty}$-topology. Let $\Gamma(N, P)$ denote the space consisting of all continuous sections of the fibre bundle $\pi_{N} \mid \Omega^{n-p+1,0}(N, P): \Omega^{n-p+1,0}(N, P) \rightarrow N$ equipped with the compactopen topology. Then there exists a continuous map $j_{\Omega}: C_{\Omega}^{\infty}(N, P) \rightarrow \Gamma(N, P)$ defined by $j_{\Omega}(f)=j^{2} f$. In dimensions $n \geq p \geq 2$ we have the homotopy principle for fold-maps in the existence level. Namely, a continuous section $s$ of $\Gamma(N, P)$ has a fold-map $f: N \rightarrow P$ such that $j^{2} f$ and $s$ are homotopic as sections of $\Gamma(N, P)$. As for this homotopy principle, we should refer to [G1], [G2], [E1], [E2] and [An3, Theorem 6] and [An4, Theorem 0.5] together with [An1, Theorem 2]. We will show how the homotopy type of the fibre $\Omega^{n-p+1,0}(n, p)$ is important for our purpose.

We denote, by $V_{n+1, p}^{\text {row }}$, the Stiefel manifold $\left(E_{p} \times O(n-p+1)\right) \backslash O(n+$ 1 ), whose elements as $p \times n$ matrices constitute, with the canonical basis of $\mathbf{R}^{n}$ and $\mathbf{R}^{p}$, the space $\mathbf{V}\left(\mathbf{R}^{n+1}, \mathbf{R}^{p}\right)$ of corresponding epimorphisms $\mathbf{R}^{n} \rightarrow$ $\mathbf{R}^{p}$. We identify both spaces throughout the paper. They have the actions of $O(p) \times O(n)$ from the lefthand side through $O(p)$ and the righthand side through $O(n) \times 1$ respectively. The group $O(p) \times O(n)$ also naturally acts on $\Omega^{n-p+1,0}(n, p)$. In order to reduce our problem of finding invariants of foldmaps to the problem concerning sections of the fiber bundle $\Omega^{n-p+1,0}(N, P)$ over $N$, we will determine the homotopy type of $\Omega^{n-p+1,0}(n, p)$ in this paper (Theorem 2.6). As a consequence of this homotopy type, we obtain a topological embedding

$$
i_{V, \Omega}: V_{n+1, p}^{r o w} \rightarrow \Omega^{n-p+1,0}(n, p)
$$

which is equivariant with respect to the actions of $O(p) \times O(n)$. Furthermore, 
if $n-p+1$ is odd, then there exists an equivariant map

$$
R_{\Omega, V}: \Omega^{n-p+1,0}(n, p) \rightarrow V_{n+1, p}^{r o w}
$$

such that $R_{\Omega, V} \circ i_{V, \Omega}$ is the identity of $V_{n+1, p}^{\text {row }}$. We provide $N$ and $P$ with Riemannian metrics. Let $\theta_{N}=N \times \mathbf{R}$. Let $\mathbf{V}\left(T N \oplus \theta_{N}, T P\right)$ denote the fiber bundle over $N \times P$ with fiber $\mathbf{V}\left(T_{x} N \oplus \mathbf{R}, T_{y} P\right)$ associated to $\mathbf{V}\left(\mathbf{R}^{n+1}, \mathbf{R}^{p}\right)$, where $(x, y)$ varies all over $(N, P)$. By the Riemannian metrics of $N$ and $P$ the structure group of $J^{2}(N, P)$ is reduced to $O(p) \times O(n)$. Let $i_{\mathbf{V}, \Omega}(N, P)$ : $\mathbf{V}\left(T N \oplus \theta_{N}, T P\right) \rightarrow \Omega^{n-p+1,0}(N, P)$ and $R_{\Omega, \mathbf{V}}(N, P): \Omega^{n-p+1,0}(N, P) \rightarrow$ $\mathbf{V}\left(T N \oplus \theta_{N}, T P\right)$ be the fiber maps associated to $i_{V, \Omega}$ and $R_{\Omega, V}$ respectively. Let $\Gamma(N, P)$ and $\Gamma\left(\mathbf{V}\left(T N \oplus \theta_{N}, T P\right)\right)$ be the space of all continuous sections of the fiber bundles $\Omega^{n-p+1,0}(N, P)$ and $\mathbf{V}\left(T N \oplus \theta_{N}, T P\right)$ over $N$ respectively equipped with the compact-open topology. Let $\Gamma\left(i_{\mathbf{V}, \Omega}\right): \Gamma(\mathbf{V}(T N \oplus$ $\left.\left.\theta_{N}, T P\right)\right) \rightarrow \Gamma(N, P)$ and $\Gamma\left(R_{\Omega, V}\right): \Gamma(N, P) \rightarrow \Gamma\left(\mathbf{V}\left(T N \oplus \theta_{N}, T P\right)\right)$ be the maps induced from the maps $i_{\mathbf{V}, \Omega}(N, P)$ and $R_{\Omega, \mathbf{V}}(N, P)$ respectively. The first result of this paper is the following theorem.

Theorem 0.1. Let $n \geq p \geq 2$. Let $N$ and $P$ be provided with Riemannian metrics. Then we have

(i) the fiber map $i_{\mathbf{V}, \Omega}(N, P): \mathbf{V}\left(T N \oplus \theta_{N}, T P\right) \rightarrow \Omega^{n-p+1,0}(N, P)$ is a topological embedding,

(ii) if $n-p+1$ is odd, then the composition $R_{\Omega, \mathbf{V}}(N, P) \circ i_{\mathbf{V}, \Omega}(N, P)$ is the identity of $\mathbf{V}\left(T N \oplus \theta_{N}, T P\right)$.

Let $\operatorname{Epi}\left(T N \oplus \theta_{N}, T P\right)$ be the fiber bundle over $N \times P$ with fiber $\operatorname{Epi}\left(T_{x} N \oplus\right.$ $\left.\theta_{N}, T_{y} P\right)$ consisting of all epimorphisms $T_{x} N \oplus \theta_{N} \rightarrow T_{y} P$. Let $\Gamma(\operatorname{Epi}(T N \oplus$ $\left.\left.\theta_{N}, T P\right)\right)$ be the space of all continuous sections of the fiber bundle $\operatorname{Epi}(T N \oplus$ $\theta_{N}, T P$ ) over $N$ equipped with the compact-open topology. Let $i_{\mathbf{V}, E p i}$ : $\mathbf{V}\left(\mathbf{R}^{n+1}, \mathbf{R}^{p}\right) \rightarrow \operatorname{Epi}\left(\mathbf{R}^{n+1}, \mathbf{R}^{p}\right)$ be the inclusion and let $i_{\mathbf{V}, E p i}(N, P): \mathbf{V}(T N \oplus$ $\left.\theta_{N}, T P\right) \rightarrow \operatorname{Epi}\left(T N \oplus \theta_{N}, T P\right)$ be the fiber homotopy equivalence associated to $i_{\mathbf{V}, E p i}$. Let $i_{\mathbf{V}, E p i}(N, P)^{-1}$ be the homotopy inverse of $i_{\mathbf{V}, E p i}(N, P)$, and let $\Gamma\left(i_{\mathbf{V}, E p i}^{-1}\right): \Gamma\left(\operatorname{Epi}\left(T N \oplus \theta_{N}, T P\right)\right) \rightarrow \Gamma\left(\mathbf{V}\left(T N \oplus \theta_{N}, T P\right)\right)$ be the map induced from $i_{\mathbf{V}, E p i}(N, P)^{-1}$. Then Theorem 0.1, [An3, Theorem 6] and [An4, Theorem 0.5] yield the following theorem.

Theorem 0.2. Let $n \geq p \geq 2$. Then any element $h \in \Gamma(\operatorname{Epi}(T N \oplus$ $\left.\left.\theta_{N}, T P\right)\right)$ has a fold map $f: N \rightarrow P$ such that $\Gamma\left(i_{\mathbf{V}, \Omega}\right) \circ \Gamma\left(i_{\mathbf{V}, E p i}^{-1}\right)(h)$ and $j^{2} f$ are homotopic as sections in $\Gamma(N, P)$. 
Let $P$ be a connected closed (resp. an oriented) smooth manifold of dimension $p$. For the study of invariants classifying fold-maps we define a fold-cobordism class of a fold-map between (resp. oriented) smooth manifolds. Namely, let $f_{i}: N_{i} \rightarrow P(i=0,1)$ be two fold-maps, where $N_{i}$ are closed (resp. oriented) smooth manifolds of dimension $n$. We say that they are (resp. oriented-) fold-cobordant when there exists a fold-map $F:(W, \partial W) \rightarrow$ $(P \times[0,1], P \times 0 \cup P \times 1)$ such that

(i) $W$ is a (resp. an oriented) smooth manifold of dimension $n+1$ with $\partial W=$ $N_{0} \cup\left(-N_{1}\right)$ and the collar of $\partial W$ is identified with $N_{0} \times[0, \varepsilon) \cup N_{1} \times(1-\varepsilon$, $1]$,

(ii) $F \mid N_{0} \times[0, \varepsilon)=f_{0} \times i d_{[0, \varepsilon)}$ and $F \mid N_{1} \times(1-\varepsilon, 1]=f_{1} \times i d_{(1-\varepsilon, 1]}$,

where $\varepsilon$ is a sufficiently small positive number. Let $\mathfrak{N}_{n}^{\text {fold }}(P)\left(\right.$ resp. $\left.\Omega_{n}^{\text {fold }}(P)\right)$ denote the set of all (resp. oriented-) fold-cobordism classes of fold-maps into $P$.

Let $\nu_{P}^{k}$ be the stable normal bundle of an embedding $P \rightarrow S^{n+k}$. Let $G_{m, \ell}$ (resp. $\widetilde{G}_{m, \ell}$ ) be the (resp. oriented) grassmann manifold of all (resp. oriented) $m$-subspaces of $\mathbf{R}^{m+\ell}$. Let $\gamma_{G_{m, \ell}}^{m}$ and $\widehat{\gamma}_{G_{m, \ell}}^{\ell}\left(\operatorname{resp} . \gamma_{\widetilde{G}_{m, \ell}}^{m}\right.$ and $\left.\widehat{\gamma}_{\widetilde{G}_{m, \ell}}^{\ell}\right)$ denote the canonical vector bundles of dimensions $m$ and $\ell$ over the space $G_{m, \ell}$ (resp. $\left.\widetilde{G}_{m, \ell}\right)$ respectively such that $\gamma_{G_{m, \ell}}^{m} \oplus \hat{\gamma}_{G_{m, \ell}}^{\ell}\left(\right.$ resp. $\left.\gamma_{\widetilde{G}_{m, \ell}}^{m} \oplus \widehat{\gamma}_{\widetilde{G}_{m, \ell}}^{\ell}\right)$ is the trivial bundle $\theta_{G_{m, \ell}}^{m+\ell}\left(\operatorname{resp} . \theta_{\widetilde{G}_{m, \ell}}^{m+\ell}\right)$. Let $T\left(\nu_{P}^{k}\right), T\left(\hat{\gamma}_{G_{m, \ell}}^{\ell}\right)$ and $T\left(\hat{\gamma}_{\widetilde{G}_{m, \ell}}^{\ell}\right)$ be the Thom spaces of $\nu_{P}^{k}, \hat{\gamma}_{G_{m, \ell}}^{\ell}$ and $\hat{\gamma}_{\widetilde{G}_{m, \ell}^{\ell}}^{\ell}$ respectively.

Theorem 0.3. $\quad$ Let $n \geq p \geq 2$ and $n-p+1$ be odd. Let $P$ be $a$ connected closed smooth manifold of dimension $p$. Let $\ell \gg n$. Then there exist the surjections

$$
\begin{aligned}
& \omega_{n, p}^{\mathfrak{N}}: \mathfrak{N}_{n}^{\text {fold }}(P) \rightarrow \lim _{k \rightarrow \infty} \pi_{n+k+\ell}\left(T\left(\nu_{P}^{k}\right) \wedge T\left(\widehat{\gamma}_{G_{n-p+1, \ell}}^{\ell}\right)\right), \\
& \omega_{n, p}^{\Omega}: \Omega_{n}^{\text {fold }}(P) \rightarrow \lim _{k \rightarrow \infty} \pi_{n+k+\ell}\left(T\left(\nu_{P}^{k}\right) \wedge T\left(\widehat{\gamma}_{\widetilde{G}_{n-p+1, \ell}^{\ell}}\right)\right) .
\end{aligned}
$$

Furthermore, we will give another invariant in a more general situation. Let $G$ refer to $G_{n, \ell}$ or $\widetilde{G}_{n, \ell}$. Let $J^{2}\left(\gamma_{G}^{n}, T P\right)$ denote the vector bundle $\operatorname{Hom}\left(\gamma_{G}^{n}, T P\right) \oplus \operatorname{Hom}\left(S^{2} \gamma_{G}^{n}, T P\right)$ over $G \times P$ with projection $p_{G}: J^{2}\left(\gamma_{G}^{n}, T P\right) \rightarrow$ $P$, where $S^{2} \gamma_{G}^{n}$ refers to the 2-fold symmetric product of $\gamma_{G}^{n}$ (see (3.1)). Let $\Omega^{n-p+1,0}\left(\gamma_{G}^{n}, T P\right)$ denote the open subbundle of $J^{2}\left(\gamma_{G}^{n}, T P\right)$ with fiber $\Omega^{n-p+1,0}(n, p)$ defined in (3.2). Consider the induced bundle $p_{G}^{*}\left(\widehat{\gamma}_{G}^{\ell}\right)$ $\mid \Omega_{\Omega^{n-p+1,0}\left(\gamma_{G}^{n}, T P\right)}$, the canonical bundle map $B_{\widehat{\gamma}^{\ell}}:\left.p_{\widetilde{G}_{n, \ell}}^{*}\left(\widehat{\gamma}_{\widetilde{G}_{n, \ell}^{\ell}}\right)\right|_{\Omega^{n-p+1,0}\left(\gamma_{G_{n, \ell}}^{n}, T P\right)}$ $\left.\rightarrow p_{G}^{*}\left(\hat{\gamma}_{G}^{\ell}\right)\right|_{\Omega^{n-p+1,0}\left(\gamma_{G}^{n}, T P\right)}$ forgetting orientations and its Thom map $T\left(B_{\widehat{\gamma}^{\ell}}\right)$. 
Theorem 0.4. Let $n \geq p \geq 2$ and $\ell \gg n$. Let $P$ be a connected smooth manifold of dimension $p$ and let $f: N \rightarrow P$ be a fold-map. Let $G$ refer to $G_{n, \ell}$ or $\widetilde{G}_{n, \ell}$, and let $P$ and $N$ be oriented when $G=\widetilde{G}_{n, \ell}$. Then $f$ determines the homotopy class $\mu_{n, p}^{G}(f)$ defined in $\lim _{\ell \rightarrow \infty} \pi_{n+\ell}\left(\left.p_{G}^{*}\left(\hat{\gamma}_{G}^{\ell}\right)\right|_{\Omega^{n-p+1,0}\left(\gamma_{G}^{n}, T P\right)}\right)$. If $P$ and $N$ are oriented in addition, then we have $\left(\lim _{\ell \rightarrow \infty} T\left(B_{\widehat{\gamma}^{\ell}}\right)\right)_{*}\left(\mu_{n, p}^{\widetilde{G}_{n, \ell}}(f)\right)=$ $\mu_{n, p}^{G_{n, \ell}}(f)$. Furthermore, every element $\alpha$ of $\lim _{\ell \rightarrow \infty} \pi_{n+\ell}\left(p_{G}^{*}\left(\widehat{\gamma}_{G}^{\ell}\right)\right.$ $\left.\left.\right|_{\Omega^{n-p+1,0}\left(\gamma_{G}^{n}, T P\right)}\right)$ has such a fold-map $f_{\alpha}: N_{\alpha} \rightarrow P$ with $\mu_{n, p}^{G}\left(f_{\alpha}\right)=\alpha$.

Here we give a brief definition of $\omega_{n, p}^{\Omega}$. By Theorem 0.1, a fold map determines an epimorphism $e_{f}: T N \oplus \theta_{N} \rightarrow T P$ covering $f$. Let $\xi$ be the kernel bundle of $e_{f}$ with induced orientation and let $\widetilde{c_{\xi}}: \xi \rightarrow \gamma_{\widetilde{G}_{n-p+1, \ell}}^{n-p+1}$ be the bundle map covering a classifying map $c_{\xi}: N \rightarrow \widetilde{G}_{n-p+1, \ell}$. Then the bundle map $b_{f}: T N \oplus \theta_{N} \rightarrow f^{*}(T P) \oplus \xi \rightarrow T P \times \gamma_{\widetilde{G}_{n-p+1, \ell}}^{n-p+1}$ covering $f \times c_{\xi}$ determines the homotopy class of a bundle map $\nu\left(b_{f}\right): \nu_{N}^{k+\ell} \rightarrow \nu_{P}^{k} \times \widehat{\gamma}_{\widetilde{G}_{n-p+1, \ell}^{\ell}}$ covering $f \times c_{\xi}$ and the map $T\left(\nu\left(b_{f}\right)\right): T\left(\nu_{N}^{k+\ell}\right) \rightarrow T\left(\nu_{P}^{k} \times \widehat{\gamma}_{\widetilde{G}_{n-p+1, \ell}^{\ell}}\right)$ by [An2, Proposition 3.3]. Let $\alpha_{N}: S^{n+k+\ell} \rightarrow T\left(\nu_{N}^{k+\ell}\right)$ be the Pontrjagin-Thom construction of an embedding $N \rightarrow S^{n+k+\ell}$. Then $\omega_{n, p}^{\Omega}(f)$ is defined to be the homotopy class of the composition $T\left(\nu\left(b_{f}\right)\right) \circ \alpha_{N}$, where $T\left(\nu_{P}^{k} \times \widehat{\gamma}_{\widetilde{G}_{n-p+1, \ell}}^{\ell}\right)$ is canonically identified with $T\left(\nu_{P}^{k}\right) \wedge T\left(\widehat{\gamma}_{\widetilde{G}_{n-p+1, \ell}^{\ell}}\right)$.

The corresponding result for $\Omega_{n}^{\text {fold }}(P)$ of Theorem 0.3 in the case $n=p$ has already been described more precisely in [An2] and [An3], while the nonoriented case was not dealt with. The homotopy type $S O(n+1)$ of $\Omega^{1,0}(n, n)$ has been important in showing the relation between fold-maps and the surgery theory, or the stable homotopy groups of spheres.

As for another line of investigation concerning the existence problem of fold-maps, we refer to the results about fold-maps of special generic type due to $[\mathrm{B}-\mathrm{R}]$, $[\mathrm{Sa}]$ and $[\mathrm{S}-\mathrm{S}]$ in low dimensions $(3,2)$ and $(4,3)$, which are closely related to the differentiable structures of manifolds.

In Section 1 we will review the fundamental properties of fold singularities and explain notations. In Section 2 we will describe the homotopy types of $\Omega^{n-p+1}(n, p)$ and $\Omega^{n-p+1,0}(n, p)$ in Theorems 2.3 and 2.6 respectively without proofs. In Section 3 we will prove Theorems $0.1,0.2,0.3$ and 0.4 by using the results in Section 2 and describe, by Theorem 0.3, differences between fold-maps and submersions. In Section 4 we will give another interpretation of $\lim _{k, \ell \rightarrow \infty} \pi_{n+k+\ell}\left(T\left(\nu_{P}^{k}\right) \wedge T\left(\widehat{\gamma}_{\widetilde{G}_{n-p+1, \ell}}\right)\right)$ by using S-dual spaces and duality maps in [Spa2] to deduce many fold-cobordism invariants in $H^{*}(P)$. In Section 5 we will prepare lemmas, which are necessary in the proof of Theorems 2.3 
and 2.6. In Section 6 we will prove Theorem 2.3. In Sections 7 and 8 we will prove Theorem 2.6 .

\section{$\S 1$. Preliminaries}

Throughout the paper all manifolds are smooth of class $C^{\infty}$. Maps are basically continuous, but may be smooth (of class $C^{\infty}$ ) if so stated. We always work in dimensions $n \geq p \geq 2$. Given a fibre bundle $\pi: E \rightarrow X$ and a subset $C$ in $X$, we denote $\pi^{-1}(C)$ by $\left.E\right|_{C}$. Let $\pi^{\prime}: F \rightarrow Y$ be another fibre bundle. A map $\tilde{b}: E \rightarrow F$ is called a fibre map over a map $b: X \rightarrow Y$ if $\pi^{\prime} \circ \tilde{b}=b \circ \pi$ holds. The restriction $\tilde{b}\left|\left(\left.E\right|_{C}\right): E\right|_{C} \rightarrow F\left(\right.$ or $\left.\left.F\right|_{b(C)}\right)$ is denoted by $\left.\tilde{b}\right|_{C}$. In particular, for a point $x \in X,\left.E\right|_{x}$ and $\left.\tilde{b}\right|_{x}$ are simply denoted by $E_{x}$ and $\tilde{b}_{x}: E_{x} \rightarrow F_{b(x)}$ respectively. When $E$ and $F$ are vector bundles, a fibrewise homomorphism, epimorphism and monomorphism $E \rightarrow F$ are simply called homomorphism, epimorphism and monomorphism respectively. The trivial bundle $X \times \mathbf{R}^{k}$ is denoted by $\theta_{X}^{n}$. In particular, $\theta_{X}^{1}$ is often written as $\theta_{X}$.

We review the fundamental properties and notations about fold singularities (see [Bo], [L] and [Ma, Section 2]). Let $J^{k}(n, p)$ denote the space consisting of all $k$-jets $j_{0}^{k} f$ of smooth map-germs $f:\left(\mathbf{R}^{n}, 0\right) \rightarrow\left(\mathbf{R}^{p}, 0\right)$. Let $L^{k}(n)$ and $L^{k}(p)$ denotes the group of all $k$-jets of local diffeomorphisms of $\left(\mathbf{R}^{n}, 0\right)$ and $\left(\mathbf{R}^{p}, 0\right)$ respectively. Then $L^{k}(n) \times L^{k}(p)$ acts on $J^{k}(n, p)$ as follows. Let $h_{1}:\left(\mathbf{R}^{n}, 0\right) \rightarrow\left(\mathbf{R}^{n}, 0\right)$ and $h_{2}:\left(\mathbf{R}^{p}, 0\right) \rightarrow\left(\mathbf{R}^{p}, 0\right)$ be local diffeomorphisms. Define the action $\left(j_{0}^{k} h_{1}, j_{0}^{k} h_{2}\right) \cdot j_{0}^{k} f=j_{0}^{k}\left(h_{2}^{-1} \circ f \circ h_{1}\right)$.

Let $\pi_{1}^{2}: J^{2}(n, p) \rightarrow J^{1}(n, p)$ be the canonical forgetting map. Let $\Sigma^{i}(n, p)$ denote the submanifold of $J^{1}(n, p)$ consisting of all 1-jets $z=j_{0}^{1} f$ such that the kernel of $d_{0} f$ is of dimension $i$. Let $\Omega^{n-p+1}(n, p)$ denote the union of $\Sigma^{n-p}(n, p)$ and $\Sigma^{n-p+1}(n, p)$ in $J^{1}(n, p)$. We denote $\left(\pi_{1}^{2}\right)^{-1}\left(\Sigma^{i}(n, p)\right)$ by the same symbol $\Sigma^{i}(n, p)$ if there is no confusion. For a 2-jet $z=j_{0}^{2} f$ of $\Sigma^{i}(n, p)$, there has been defined the second intrinsic derivative $d_{0}^{2} f: T_{0} \mathbf{R}^{n} \rightarrow \operatorname{Hom}\left(\operatorname{Ker}\left(d_{0} f\right)\right.$, $\left.\operatorname{Cok}\left(d_{0} f\right)\right)$. Let $\Sigma^{i, j}(n, p)$ denote the submanifold of $J^{2}(n, p)$ consisting of all jets $z=j_{0}^{2} f$ such that $\operatorname{dim}\left(\operatorname{Ker}\left(d_{0} f\right)\right)=i$ and $\operatorname{dim}\left(\operatorname{Ker}\left(d_{0}^{2} f \mid \operatorname{Ker}\left(d_{0} f\right)\right)\right)=j$. We say that a jet of $\Sigma^{n-p+1,0}(n, p)$ has the Boardman symbol $(n-p+1,0)$. Let $\Omega^{n-p+1,0}(n, p)$ denote the union of $\Sigma^{n-p}(n, p)$ and $\Sigma^{n-p+1,0}(n, p)$ in $J^{2}(n, p)$.

We note that with the canonical bases of $\mathbf{R}^{n}$ and $\mathbf{R}^{p}, J^{2}(n, p)$ is identified with $\operatorname{Hom}\left(\mathbf{R}^{n}, \mathbf{R}^{p}\right) \oplus \operatorname{Hom}\left(S^{2} \mathbf{R}^{n}, \mathbf{R}^{p}\right)$, by considering the Taylor expansion of $f$, where $S^{2} \mathbf{R}^{n}$ is the 2-fold symmetric product of $\mathbf{R}^{n}$. Furthermore, throughout the paper, we always identify $\operatorname{Hom}\left(\mathbf{R}^{n}, \mathbf{R}^{p}\right)$ with the space $M_{p \times n}$ of all $p \times n$ matrices and identify $\operatorname{Hom}\left(S^{2} \mathbf{R}^{n}, \mathbf{R}^{p}\right)$ with the space of all $p$-tuples of $n \times n$ symmetric matrices. For subspaces $V$ and $W, V \bigcirc W$ or $S^{2} V$ denotes 
the symmetric product in $S^{2} \mathbf{R}^{n}$. In this paper we often express an element of $J^{2}(n, p)$ as $(\alpha, \beta)$ where $\alpha \in \operatorname{Hom}\left(\mathbf{R}^{n}, \mathbf{R}^{p}\right)$ and $\beta \in \operatorname{Hom}\left(S^{2} \mathbf{R}^{n}, \mathbf{R}^{p}\right)$. For a subspace $V$ in $\mathbf{R}^{p}$, let $\operatorname{pr}(V)$ be the orthogonal projection of $\mathbf{R}^{p}$ onto $V$. For an element $(\alpha, \beta) \in \Sigma^{n-p+1}(n, p)$, let $\beta_{\alpha}: S^{2} \operatorname{Ker}(\alpha) \rightarrow \operatorname{Im}(\alpha)^{\perp}$ denote a homomorphism defined by

$$
\beta_{\alpha}=\operatorname{pr}\left(\operatorname{Im}(\alpha)^{\perp}\right) \circ\left(\beta \mid S^{2} \operatorname{Ker}(\alpha)\right),
$$

where the symbol $\perp$ refers to the orthogonal complement. Then $\alpha \in J^{1}(n, p)$ lies in $\Sigma^{n-p+1}(n, p)$ if and only if $\operatorname{dim} \operatorname{Ker}(\alpha)=n-p+1$, and $(\alpha, \beta) \in$ $\Sigma^{n-p+1}(n, p)$ lies in $\Sigma^{n-p+1,0}(n, p)$ if and only if $\beta_{\alpha}$ is a non-singular quadratic form.

For a subset $X$ and an element $x$, an equivalence class of $x$ is usually expressed as $[x]$.

\section{$\S 2 . \quad H o m o t o p y$ Types}

In this section we describe the homotopy types of $\Omega^{n-p+1}(n, p)$ and $\Omega^{n-p+1,0}(n, p)$ in dimensions $n \geq p \geq 2$.

Let $X$ and $Y$ be spaces and let $G$ be a Lie group. If $G$ acts on $X$ from the right-hand (resp. left-hand) side, then the orbit space is denoted by $X / G$ (resp. $G \backslash X)$. If $G$ acts on $X$ and $Y$ from the right-hand and left-hand sides respectively, then $G$ acts on $X \times Y$ by $g \cdot(x, y)=\left(x g^{-1}, g y\right)$. We define the twisted product of $X$ and $Y$ to be the orbit space $X \times_{G} Y$ of this action and denote its element by $[x, y]$ for $x \in X$ and $y \in Y$. Namely, we have $[x, y]=\left[x g^{-1}, g y\right]$.

Let $A_{1}, \ldots, A_{s}$ be the real square matrices of degree $i_{1}, \ldots, i_{s}$ respectively. The matrix of the form

$$
\left(\begin{array}{lll}
A_{1} & & \mathbf{0} \\
& \ddots & \\
& & \\
\mathbf{0} & & A_{s}
\end{array}\right)
$$

will be denoted by $A_{1} \dot{+} \cdots \dot{+} A_{s}$. The diagonal matrix of degree $k$ with diagonal components $\mathbf{d}=\left(d_{1}, \ldots, d_{k}\right)$ will be denoted by $\Delta(\mathbf{d})$. The unit matrix of degree $k$ is denoted by $E_{k}$.

Let $O(k)$ and $S O(k)$ be the orthogonal group and the rotation group of degree $k$ respectively. For a matrix $M=\left(m_{i j}\right) \in O(k)$, the $i$-th row and column vectors are denoted by $\mathbf{m}_{i}$ and $\overline{\mathbf{m}}_{i}$ respectively. Let $M(i, j)$ and $M\left(\begin{array}{l}i \\ j\end{array}\right)$ be the 
minor-matrices

$$
\left(\overline{\mathbf{m}}_{i}, \ldots, \overline{\mathbf{m}}_{j}\right) \text { and }\left(\begin{array}{c}
\mathbf{m}_{i} \\
\vdots \\
\mathbf{m}_{j}
\end{array}\right)
$$

respectively. Let $k \geq h$. Throughout the paper the Stiefel manifolds $\left(E_{h} \times O(k-\right.$ $h)) \backslash O(k)$ and $O(k) /\left(E_{h} \times O(k-h)\right)$ are canonically identified with the space consisting of all $k \times h$-matrices $M(1, h)$ and $h \times k$-matrices $M\left(\begin{array}{l}1 \\ h\end{array}\right)$ respectively, where $M$ varies in $O(k)$. Let $I$ be the interval $[0,1]$. For $b \in I$, let $\mathbf{d}_{b}$ be the diagonal components $(1, \ldots, 1, b)$, where the degree should be relevant to the arguments. Let $\Delta\left(\mathbf{d}_{b}\right)$ be the diagonal matrix with diagonal components $\mathbf{d}_{b}$. In this paper $E_{h} \times O(0)$ and $O(h) \times O(0)$ refers to $E_{h}$ and $O(h)$ respectively.

We consider the following action of $O(p) \times O(n)$ on $J^{k}(n, p)$. We regard $O \in O(p)$ and $U \in O(n)$ as linear maps, $\mathbf{R}^{p} \rightarrow \mathbf{R}^{p}$ and $\mathbf{R}^{n} \rightarrow \mathbf{R}^{n}$ respectively. Then define the action of $(O, U)$ on a jet $z=j_{0}^{k} f$ by

$$
(O, U) \cdot z=j_{0}^{k}\left(O \circ f \circ U^{-1}\right) .
$$

Now we describe the homotopy types of the spaces $\Omega^{n-p+1}(n, p)$ and $\Omega^{n-p+1,0}(n, p)$ in dimensions $n \geq p \geq 2$.

Throughout the paper we denote, by $V_{n, p}^{\text {row }}$, the Stiefel manifold $\left(E_{p} \times\right.$ $O(n-p)) \backslash O(n)$.

Case I: $\Omega^{n-p+1}(n, p)$. We first define several actions. The actions of $O(p-1)$ and $O(1)$ on $O(p)$ and $O(n)$ are defined as follows. For elements $G \in O(p-1),(\delta) \in O(1), S \in O(p)$ and $M \in O(n)$, we set

$$
\begin{array}{rlrl}
G \cdot S & =S\left({ }^{t} G \dot{+}(1)\right), & G \cdot M & =\left(G \dot{+} E_{n-p+1}\right) M, \\
(\delta) \cdot S & =S\left(E_{p-1} \dot{+}(\delta)\right), \quad(\delta) \cdot M=\left(E_{p-1} \dot{+}(\delta) \dot{+} E_{n-p}\right) M .
\end{array}
$$

We define the twisted products $\mathfrak{k}(n, p), K(n, p, b)$ for $0 \leq b \leq 1$ and $\Sigma K(n, p)$ defined by

$$
\begin{aligned}
\mathfrak{k}(n, p) & =O(p) \times_{O(p-1) \times O(1)}\left\{\left(E_{p} \times O(n-p)\right) \backslash O(n)\right\}, \\
K(n, p, b) & =\mathfrak{k}(n, p) \times b, \\
\Sigma K(n, p) & =\left\{O(p) /\left(E_{p-1} \times O(1)\right)\right\} \times_{O(p-1)}\left\{\left(E_{p-1} \times O(n-p+1)\right) \backslash O(n)\right\} .
\end{aligned}
$$

An element of $K(n, p, b), \Sigma K(n, p)$ or $V_{n, p}^{\text {row }}$ can be expressed by $\left[S, M\left(\begin{array}{l}1 \\ p\end{array}\right), b\right]$, $\left[S, M\left(\begin{array}{c}1 \\ p-1\end{array}\right)\right]$ or $M\left(\begin{array}{l}1 \\ p\end{array}\right)$ respectively, where $S \in O(p)$ and $M \in O(n)$. 
Remark 2.1. Let $[z]=\left[S, M\left(\begin{array}{l}1 \\ p\end{array}\right), b\right]$, or $\left[S, M\left(\begin{array}{c}1 \\ p-1\end{array}\right)\right]$, and $\left[z^{\prime}\right]=\left[S^{\prime}, M^{\prime}\left(\begin{array}{l}1 \\ p\end{array}\right)\right.$, $b]$, or $\left[S^{\prime}, M^{\prime}\left(\begin{array}{c}1 \\ p-1\end{array}\right)\right]$ be elements of $K(n, p, b)$, and $\Sigma K(n, p)$ respectively. Then $[z]=\left[z^{\prime}\right]$ if and only if there exist matrices $G \in O(p-1), L \in O(n-p)$ and $L_{n-p+1} \in O(n-p+1)$ such that

(i) $S^{\prime}=S\left({ }^{t} G \dot{+}(\delta)\right)$ and $M^{\prime}=(G \dot{+}(\delta) \dot{+} L) M$ for $b>0$,

(ii) $S^{\prime}=S\left({ }^{t} G \dot{+}(\delta)\right)$ and $M^{\prime}=\left(G \dot{+} E_{n-p+1}\right)\left(E_{p-1} \dot{+} L_{n-p+1}\right) M$ for $b=0$.

There exist the continuous surjections

$$
\begin{aligned}
& \rho_{n, p, \Sigma}: K(n, p, 0) \rightarrow \Sigma K(n, p), \\
& \rho_{n, p, R}: K(n, p, 1) \rightarrow V_{n, p}^{r o w}
\end{aligned}
$$

defined by $\rho_{n, p, \Sigma}\left(\left[S, M\left(\begin{array}{l}1 \\ p\end{array}\right), 0\right]\right)=\left[S, M\left(\begin{array}{c}1 \\ p-1\end{array}\right)\right]$ and $\rho_{n, p, R}\left(\left[S, M\left(\begin{array}{l}1 \\ p\end{array}\right), 1\right]\right)=S M\left(\begin{array}{l}1 \\ p\end{array}\right)$. It is easily seen that these maps are well defined. We define the space $K(n, p)$ to be the quotient space obtained from the disjoint union

$$
\Sigma K(n, p) \bigcup \mathfrak{k}(n, p) \times I \bigcup V_{n, p}^{r o w}
$$

by identifying $K(n, p, 0)$ with $\Sigma K(n, p)$ by $\rho_{n, p, \Sigma}$ and $K(n, p, 1)$ with $V_{n, p}^{\text {row }}$ by $\rho_{n, p, R}$ respectively. Namely, we identify $\left[S, M\left(\begin{array}{l}1 \\ p\end{array}\right), 0\right]=\left[S, M\left({ }_{p-1}^{1}\right)\right]$ and $\left[S, M\left(\begin{array}{l}1 \\ p\end{array}\right), 1\right]=S M\left(\begin{array}{l}1 \\ p\end{array}\right)$. Then there exists a continuous map

$$
i_{n, p}: K(n, p) \rightarrow \Omega^{n-p+1}(n, p)
$$

defined by $i_{n, p}\left(\left[S, M\left(\begin{array}{l}1 \\ p\end{array}\right), b\right]\right)=S \Delta\left(\mathbf{d}_{b}\right) M\left(\begin{array}{l}1 \\ p\end{array}\right)$. We define the action of $O(p) \times$ $O(n)$ on $K(n, p)$ by

$$
(O, U) \cdot\left[S, M\left(\begin{array}{l}
1 \\
p
\end{array}\right), b\right]=\left[O S, M\left(\begin{array}{l}
1 \\
p
\end{array}\right) U^{-1}, b\right] .
$$

Lemma 2.2. The map $i_{n, p}$ is well defined, and is equivariant with respect to the actions of $O(p) \times O(n)$.

Proof. Suppose that $[z]=\left[S, M\left(\begin{array}{l}1 \\ p\end{array}\right), b\right]$ and $\left[z^{\prime}\right]=\left[S^{\prime}, M^{\prime}\left(\begin{array}{l}1 \\ p\end{array}\right), b\right]$ in $K(n, p, b)$ as given in Remark 2.1. If $[z]=\left[z^{\prime}\right]$, then we have $S \Delta\left(\mathbf{d}_{b}\right) M\left(\begin{array}{l}1 \\ p\end{array}\right)=$ $S^{\prime} \Delta\left(\mathbf{d}_{b}\right) M^{\prime}\left(\begin{array}{l}1 \\ p\end{array}\right)$, and hence, $i_{n, p}([\mathbf{z}])=i_{n, p}\left(\left[\mathbf{z}^{\prime}\right]\right)$.

If $(O, U) \in O(p) \times O(n)$, then we have by $(2.1)$

$$
i_{n, p}((O, U) \cdot[\mathbf{z}])=O S \Delta\left(\mathbf{d}_{b}\right) M\left(\begin{array}{l}
1 \\
p
\end{array}\right) U^{-1}=(O, U) \cdot i_{n, p}([\mathbf{z}]) .
$$

This shows the lemma.

The following theorem will be proved in Section 6 . 
Theorem 2.3. $\quad$ The map $i_{n, p}$ is an equivariant topological embedding. There exists a deformation retraction $R_{\lambda}$ of $\Omega^{n-p+1}(n, p)$ to $i_{n, p}(K(n, p))$ such that

(i) $R_{\lambda}$ preserves $\Sigma^{n-p}(n, p)$ and $\Sigma^{n-p+1}(n, p)$ respectively,

(ii) the restriction $R_{\lambda} \mid \Sigma^{n-p+1}(n, p)$ is a deformation retraction of $\Sigma^{n-p+1}(n, p)$ to $i_{n, p}(\Sigma K(n, p))$.

Case II: $\Omega^{n-p+1,0}(n, p)$. Let $c, d$ and $\sigma$ always denote the integers such that $c \geq d \geq 0, c+d=n-p+1$ and $\sigma=c-d$. We consider the actions in (2.2) and the actions of $O(n-p)$ on $O(n-p+1)$ and $O(n)$ defined as follows. For elements $L \in O(n-p), T \in O(n-p+1)$ and $M \in O(n)$, we define

$$
L \cdot T=T\left((1) \dot{+}{ }^{t} L\right), \quad L \cdot M=\left(E_{p} \dot{+} L\right) M .
$$

Next we define the action of an element $G \in O(p-1)$ on an element $[S, T, M] \in$ $O(p) \times\left\{((O(c) \times O(d)) \backslash O(n-p+1)) \times_{1 \times O(p-1)} O(n)\right\}$ by

$$
G \cdot[S, T, M]=\left[S\left({ }^{t} G \dot{+}(1)\right), T,\left(G \dot{+} E_{n-p+1}\right) M\right] .
$$

If $\sigma=0$ and $n-p+1=2 c$, then we consider two other actions of $O(1)$. Whenever we deal with these actions of $O(1)$, we denote $O(1)$ by $\widetilde{O(1)}$ to emphasize these exceptional actions. The action of an element $(\delta) \in \widetilde{O(1)}$ on an element $[S, T, M] \in O(p) \times{ }_{O(p-1)}(((O(c) \times O(c)) \backslash O(2 c)) \times O(n))$ is defined by

(1) $\cdot[S, T, M]=[S, T, M]$,

$(-1) \cdot[S, T, M]=\left[S\left(E_{p-1} \dot{+}(-1)\right),\left(\begin{array}{cc}0 & E_{c} \\ E_{c} & 0\end{array}\right) T,\left(E_{p-1} \dot{+}(-1) \dot{+} E_{n-p}\right) M\right]$.

We define another action of $\widetilde{O(1)}$ on $O(p) \times_{O(p-1) \times 1}\left(\left(E_{p-1} \times O(c) \times O(c)\right) \backslash O(n)\right)$ as follows. For elements $(-1) \in \widetilde{O(1)}$ and $\left[S, M\left(\begin{array}{l}p \\ n\end{array}\right)\right] \in O(p) \times_{O(p-1) \times 1}\left(\left(E_{p-1} \times\right.\right.$ $O(c) \times O(c)) \backslash O(n))$, define

$$
\begin{aligned}
(1) \cdot[S, M] & =[S, M] \\
(-1) \cdot[S, M] & =\left[S\left(E_{p-1} \dot{+}(-1)\right),\left(E_{p-1} \dot{+}\left(\begin{array}{ll}
0 & E_{c} \\
E_{c} & 0
\end{array}\right)\right) M\right] .
\end{aligned}
$$


These actions in (2.9) and (2.10) are well defined. Indeed, for $T_{1}, T_{2} \in O(c)$ we have

$$
\begin{aligned}
& (-1) \cdot\left[S,\left(E_{p-1} \dot{+}\left(\begin{array}{cc}
T_{1} & 0 \\
0 & T_{2}
\end{array}\right)\right) M\right] \\
& =\left[S\left(E_{p-1} \dot{+}(-1)\right),\left(E_{p-1} \dot{+}\left(\begin{array}{cc}
0 & E_{c} \\
E_{c} & 0
\end{array}\right)\left(\begin{array}{cc}
T_{1} & 0 \\
0 & T_{2}
\end{array}\right)\right) M\right] \\
& =\left[S\left(E_{p-1} \dot{+}(-1)\right),\left(E_{p-1} \dot{+}\left(\begin{array}{cc}
0 & T_{2} \\
T_{1} & 0
\end{array}\right)\right) M\right] \\
& =\left[S\left(E_{p-1} \dot{+}(-1)\right),\left(E_{p-1} \dot{+}\left(\begin{array}{cc}
T_{2} & 0 \\
0 & T_{1}
\end{array}\right)\left(\begin{array}{cc}
0 & E_{c} \\
E_{c} & 0
\end{array}\right)\right) M\right] \\
& =\left[S\left(E_{p-1} \dot{+}(-1)\right),\left(E_{p-1} \dot{+}\left(\begin{array}{cc}
0 & E_{c} \\
E_{c} & 0
\end{array}\right)\right) M\right] \\
& =(-1) \cdot[S, M] \text {. }
\end{aligned}
$$

For $0<\sigma \leq n-p+1$ and $b \in I$, let $\mathfrak{K}(n, p, \sigma), \mathcal{K}(n, p, \sigma, b)$ and $\Sigma \mathcal{K}(n, p, \sigma)$ be the spaces defined by

$$
\begin{aligned}
\mathfrak{K}(n, p, \sigma) & =O(p) \times_{O(p-1) \times 1}\left\{((O(c) \times O(d)) \backslash O(n-p+1)) \times_{1 \times O(n-p)} O(n)\right\}, \\
\mathcal{K}(n, p, \sigma, b) & =\mathfrak{K}(n, p, \sigma) \times b, \\
\Sigma \mathcal{K}(n, p, \sigma) & =O(p) \times_{O(p-1) \times 1}\left\{\left(E_{p-1} \times O(c) \times O(d)\right) \backslash O(n)\right\} .
\end{aligned}
$$

For $\sigma=0, n-p+1=2 c(c=d)$ and $b \in I$, we define the spaces $\mathfrak{K}(n, p, 0)$, $\mathcal{K}(n, p, 0, b)$ and $\Sigma \mathcal{K}(n, p, 0)$ to be

$$
\begin{aligned}
\mathfrak{K}(n, p, 0) & =O(p) \times_{O(p-1) \times \widetilde{O(1)}}\left\{((O(c) \times O(c)) \backslash O(2 c)) \times_{1 \times O(n-p)} O(n)\right\}, \\
\mathcal{K}(n, p, 0, b) & =\mathfrak{K}(n, p, 0) \times b, \\
\Sigma \mathcal{K}(n, p, 0) & =O(p) \times_{O(p-1) \times \widetilde{O(1)}}\left\{\left(E_{p} \times O(c) \times O(c)\right) \backslash O(n)\right\} .
\end{aligned}
$$

An element of $\mathcal{K}(n, p, \sigma, b)$ or $\Sigma \mathcal{K}(n, p, \sigma)$ will be expressed by $[S, T, M, \sigma, b]$ or $[S, M, \sigma]$ respectively, where $S \in O(p), T \in O(n-p+1), M \in O(n)$ and $b \in I$.

The following remark follows from (2.2) and (2.7) to (2.12).

Remark 2.4. Let $[z]=[S, T, M, \sigma, b]$, or $[S, M, \sigma]$, and $\left[z^{\prime}\right]=\left[S^{\prime}, T^{\prime}, M^{\prime}\right.$, $\sigma, b]$, or $\left[S^{\prime}, M^{\prime}, \sigma\right]$ be elements of $\mathcal{K}(n, p, \sigma, b)$ or $\Sigma \mathcal{K}(n, p, \sigma)$. Then $[z]=\left[z^{\prime}\right]$ in $\mathcal{K}(n, p, \sigma, b)$ if and only if there exist matrices $G \in O(p-1), L \in O(n-p)$, $T_{1} \in O(c)$ and $T_{2} \in O(d)$ such that 
Case (i): $\sigma>0$ and $0<b<1$,

$$
\begin{aligned}
S^{\prime} & =S\left({ }^{t} G \dot{+}(1)\right), \quad T^{\prime}=\left(T_{1} \dot{+} T_{2}\right) T\left((1)+{ }^{t} L\right) \quad \text { and } \\
M^{\prime} & =\left(G \dot{+} E_{n-p+1}\right)\left(E_{p} \dot{+} L\right) M,
\end{aligned}
$$

Case (ii): $\sigma>0$ and $b=0$,

$$
S^{\prime}=S\left({ }^{t} G \dot{+}(1)\right) \quad \text { and } \quad M^{\prime}=\left(G \dot{+} T_{1} \dot{+} T_{2}\right) M,
$$

Case (iii): $\sigma=0$ and $0<b<1$, either

$$
\begin{aligned}
S^{\prime} & =S\left({ }^{t} G \dot{+}(1)\right), \quad T^{\prime}=\left(T_{1} \dot{+} T_{2}\right) T\left((1) \dot{+}{ }^{t} L\right) \quad \text { and } \\
M^{\prime} & =\left(G \dot{+} E_{n-p+1}\right)\left(E_{p} \dot{+} L\right) M,
\end{aligned}
$$

or

$$
\begin{aligned}
S^{\prime} & =S\left({ }^{t} G \dot{+}(-1)\right), \quad T^{\prime}=\left(\begin{array}{ll}
0 & E_{c} \\
E_{c} & 0
\end{array}\right)\left(T_{1} \dot{+} T_{2}\right) T\left((-1) \dot{+}{ }^{t} L\right) \quad \text { and } \\
M^{\prime} & =(G \dot{+}(-1) \dot{+} L) M .
\end{aligned}
$$

Case (iv): $\sigma=0$ and $b=0$, either

$$
S^{\prime}=S\left({ }^{t} G \dot{+}(1)\right) \quad \text { and } \quad M^{\prime}=\left(G \dot{+} T_{1} \dot{+} T_{2}\right) M,
$$

or

$$
S^{\prime}=S\left({ }^{t} G \dot{+}(-1)\right) \quad \text { and } \quad M^{\prime}=\left(G \dot{+}\left(\begin{array}{ll}
0 & E_{c} \\
E_{c} & 0
\end{array}\right)\right)\left(T_{1}+T_{2}\right) M .
$$

There exists the continuous surjections

$$
\begin{aligned}
& \bar{\rho}_{n, p, \Sigma}: \mathcal{K}(n, p, \sigma, 0) \rightarrow \Sigma \mathcal{K}(n, p, \sigma), \\
& \bar{\rho}_{n, p, R}: \mathcal{K}(n, p, \sigma, 1) \rightarrow V_{n, p}^{r o w}
\end{aligned}
$$

defined by

$$
\begin{aligned}
& \bar{\rho}_{n, p, \Sigma}([S, T, M, \sigma, 0])=\left[S,\left(E_{p-1}+T\right) M, \sigma\right], \\
& \bar{\rho}_{n, p, R}([S, T, M, \sigma, 1])=S\left(\left(\left(E_{p-1}+T\right) M\right)\left(\begin{array}{l}
1 \\
p
\end{array}\right)\right)
\end{aligned}
$$

respectively. It is easy to see that these maps are well defined. 
We define the space $\mathcal{K}(n, p, \sigma)$ to be the quotient space obtained from the disjoint union

$$
\Sigma \mathcal{K}(n, p, \sigma) \bigcup \mathfrak{K}(n, p, \sigma) \times I \bigcup V_{n, p}^{r o w}
$$

by identifying $\mathcal{K}(n, p, \sigma, 0)$ with $\Sigma \mathcal{K}(n, p, \sigma)$ by $\bar{\rho}_{n, p, \Sigma}$ and $\mathcal{K}(n, p, \sigma, 1)$ with $V_{n, p}^{\text {row }}$ by $\bar{\rho}_{n, p, R}$. Namely, we identify $[S, T, M, \sigma, 0]=\left[S,\left(E_{p-1}+T\right) M, \sigma\right]$ and $[S, T, M, \sigma, 1]=S\left(E_{p-1}+T\right) M\left(\begin{array}{l}1 \\ p\end{array}\right)$. We define the space $\mathcal{K}(n, p)$ to be the quotient space obtained from the union

$$
\bigcup_{d=0}^{[(n-p+1) / 2]} \mathcal{K}(n, p, n-p+1-2 d)
$$

by the identification such that all subspaces $V_{n, p}^{\text {row }}$ in $\mathcal{K}(n, p, n-p+1-2 d), 0 \leq$ $d \leq[(n-p+1) / 2]$ are pasted each other by the identity of $V_{n, p}^{r o w}$. Furthermore, we define $\Sigma \mathcal{K}(n, p)$ to be the union

$$
\bigcup_{d=0}^{[(n-p+1) / 2]} \Sigma \mathcal{K}(n, p, n-p+1-2 d) .
$$

There exists a continuous map

$$
\mathcal{I}_{n, p}: \mathcal{K}(n, p) \rightarrow \Omega^{n-p+1,0}(n, p)
$$

defined as follows. Let $[\mathbf{z}]$ represent an element $[S, T, M, \sigma, b]$ or $[S, M, \sigma]$ of $\mathcal{K}(n, p, \sigma)$. Let $\overline{\mathbf{s}}_{p}=S \mathbf{e}_{p}$. Define $\alpha([\mathbf{z}])$ and $\beta([\mathbf{z}])$ to be the elements of $\Omega^{n-p+1}(n, p)$ and $\operatorname{Hom}\left(S^{2} \mathbf{R}^{n}, \mathbf{R}^{p}\right)$ defined by

$$
\begin{aligned}
\alpha([\mathbf{z}]) & =S \Delta\left(\mathbf{d}_{b}\right) M\left(\begin{array}{l}
1 \\
p
\end{array}\right), \\
\beta([\mathbf{z}])(\mathbf{x}, \mathbf{y}) & =\sqrt{1-b^{2}}\left\{{ }^{t} \mathbf{x}^{t} M\left(\begin{array}{l}
p \\
n
\end{array}\right)^{t} T\left(E_{c} \dot{+}\left(-E_{d}\right)\right) T M\left(\begin{array}{l}
p \\
n
\end{array}\right) \mathbf{y}\right\} \overline{\mathbf{s}}_{p},
\end{aligned}
$$

respectively, where if $b=0$, then $T$ should be replaced by $E_{n-p+1}$. We have the following properties:

(i) If $b=1$, then $\beta([\mathbf{z}])=\mathbf{0}$.

(ii) For $0 \leq b<1$, let $K_{\alpha([\mathbf{z}])}$ denote the subspace generated by ${ }^{t} \mathbf{m}_{p}, \ldots,{ }^{t} \mathbf{m}_{n}$. If $\mathbf{x} \in\left(K_{\alpha([\mathbf{z}])}\right)^{\perp}$, or $\mathbf{y} \in\left(K_{\alpha([\mathbf{z}])}\right)^{\perp}$, then $\beta([\mathbf{z}])(\mathbf{x}, \mathbf{y})=\mathbf{0}$.

(iii) $\beta([\mathbf{z}])$ is non-singular on $S^{2}\left(K_{\alpha([\mathbf{z}])}\right)$.

If we define the map $\mathcal{I}_{n, p}$ by

$$
\mathcal{I}_{n, p}([\mathbf{z}])=(\alpha([\mathbf{z}]), \beta([\mathbf{z}])),
$$


then this is the map into $\Omega^{n-p+1,0}(n, p)$. We define the action of $O(p) \times O(n)$ on $\mathcal{K}(n, p)$ by

$$
(O, U) \cdot[S, T, M, \sigma, b]=\left[O S, T, M U^{-1}, \sigma, b\right] .
$$

Lemma 2.5. The map $\mathcal{I}_{n, p}$ is well defined and equivariant with respect to the action of $O(p) \times O(n)$.

Proof. The fact that $\alpha([\mathbf{z}])$ is well defined and equivariant is proved analogously as in the proof of Lemma 2.3 .

We show that $\beta([S, T, M, \sigma, b])$ is well defined. Suppose that

(i) $[S, T, M, \sigma, b]=\left[S^{\prime}, M^{\prime}, T^{\prime}, \sigma, b\right]$ in $\mathcal{K}(n, p, \sigma, b)$ or

(ii) $[S, M, \sigma]=\left[S^{\prime}, M^{\prime}, \sigma\right]$ in $\Sigma \mathcal{K}(n, p, \sigma)$.

In the Case (i), by Remark 2.5, there are matrices $G \in O(p-1), L \in$ $O(n-p), T_{2} \in O(c)$ and $T_{3} \in O(d)$ such that

$$
\begin{aligned}
& S^{\prime}=S\left({ }^{t} G \dot{+}(1)\right), \quad T^{\prime}=\left(T_{2} \dot{+} T_{3}\right) T\left((1) \dot{+}{ }^{t} L\right) \\
& \text { and } M^{\prime}=\left(G \dot{+} E_{n-p+1}\right)\left(E_{p} \dot{+} L\right) M \quad \text { for } \sigma>0, \\
& S^{\prime}=S\left({ }^{t} G \dot{+}(-1)\right), \quad T^{\prime}=\left(T_{2} \dot{+} T_{3}\right)\left(\begin{array}{cc}
0 & E_{c} \\
E_{c} & 0
\end{array}\right) T\left((-1) \dot{+}{ }^{t} L\right) \\
& \text { and } M^{\prime}=\left(G \dot{+} E_{n-p+1}\right)\left(E_{p-1} \dot{+}(-1) \dot{+} L\right) M \text { for } \sigma=0 .
\end{aligned}
$$

Hence, the space generated by ${ }^{t} \mathbf{m}_{p}, \ldots,{ }^{t} \mathbf{m}_{n}$ is well defined and $S \mathbf{e}_{p}=$ $S^{\prime} \mathbf{e}_{p}$. Furthermore, we have

$$
{ }^{t} M\left(\begin{array}{l}
p \\
n
\end{array}\right)^{t} T\left(E_{c} \dot{+}\left(-E_{d}\right)\right) T M\left(\begin{array}{l}
p \\
n
\end{array}\right)={ }^{t} M^{\prime}\left(\begin{array}{l}
p \\
n
\end{array}\right){ }^{t} T^{\prime}\left(E_{c} \dot{+}\left(-E_{d}\right)\right) T^{\prime} M^{\prime}\left(\begin{array}{l}
p \\
n
\end{array}\right) .
$$

Therefore, we have $\beta([\mathbf{z}])=\beta\left(\left[\mathbf{z}^{\prime}\right]\right)$. The Case (ii) is a special case of the Case (i) and can be proved independently as in (i).

Next we show that $\beta: S^{2} \mathbf{R}^{n} \rightarrow \mathbf{R}^{p}$ is equivariant. We have

$$
\begin{aligned}
\beta((O, U) \cdot[\mathbf{z}])(\mathbf{x}, \mathbf{y}) & =\beta\left(\left[O S, T, M U^{-1}, \sigma, b\right]\right)(\mathbf{x}, \mathbf{y}) \\
& =\sqrt{1-b^{2}}\left\{{ }^{t} \mathbf{x} U^{t} M\left(\begin{array}{l}
p \\
n
\end{array}\right)^{t} T\left(E_{c} \dot{+}\left(-E_{d}\right)\right) T M^{t} U\left(\begin{array}{l}
p \\
n
\end{array}\right) \mathbf{y}\right\} O \overline{\mathbf{s}}_{p} \\
& =\sqrt{1-b^{2}}\left\{\left({ }^{t}\left({ }^{t} U \mathbf{x}\right)^{t} M\left(\begin{array}{l}
p \\
n
\end{array}\right)^{t} T\left(E_{c} \dot{+}\left(-E_{d}\right)\right) T M\left(\begin{array}{l}
p \\
n
\end{array}\right)^{t} U \mathbf{y}\right\} O \overline{\mathbf{s}}_{p}\right. \\
& =O \beta([\mathbf{z}])\left(U^{-1} \mathbf{x}, \mathbf{U}^{-1} \mathbf{y}\right) \\
& =((O, U) \beta([\mathbf{z}]))(\mathbf{x}, \mathbf{y}) .
\end{aligned}
$$

This shows the lemma.

Now we are ready to state the follwing theorem, which will be proved in Sections 6 and 8 . 
Theorem 2.6. Let $n \geq p \geq 2$. The map $\mathcal{I}_{n, p}$ is an equivariant topological embedding. There exists a deformation retraction $\mathcal{R}_{\lambda}$ of $\Omega^{n-p+1,0}(n, p)$ to $\mathcal{I}_{n, p}(\mathcal{K}(n, p))$ such that

(i) $\mathcal{R}_{\lambda}$ preserves $\Sigma^{n-p}(n, p)$ and $\Sigma^{n-p+1,0}(n, p)$ respectively,

(ii) the restriction $R_{\lambda} \mid \Sigma^{n-p+1,0}(n, p)$ is a deformation retraction of $\Sigma^{n-p+1,0}(n, p)$ to $\mathcal{I}_{n, p}(\Sigma \mathcal{K}(n, p))$.

We consider the action of $O(p) \times O(n)$ on $V_{n+1, p}^{\text {row }}$ defined by

$$
(O, U) \cdot M_{p \times(n+1)}=O M_{p \times(n+1)}\left(U^{-1} \dot{+}(1)\right) .
$$

We now show that $\mathcal{K}(n, p, n-p+1)$ is homeomorphic to $V_{n+1, p}^{\text {row }}$.

Proposition 2.7. Let $n \geq p \geq 2$. Then there exists a homeomorphim $j_{\mathcal{K}, V}: \mathcal{K}(n, p, n-p+1) \rightarrow V_{n+1, p}^{\text {row }}$, which is equivariant with respect to the actions of $O(p) \times O(n)$.

Proof. Let

$$
j_{\mathcal{K}, V}: \mathcal{K}(n, p, n-p+1, b) \rightarrow V_{n+1, p}^{\text {row }},
$$

be the map defined by

$$
j_{\mathcal{K}, V}([S, T, M, n-p+1, b])=S\left(\begin{array}{cc}
\mathbf{m}_{1} & 0 \\
\vdots & \vdots \\
\mathbf{m}_{p-1} & 0 \\
b \mathbf{m}_{p} & \sqrt{1-b^{2}}
\end{array}\right) \quad \text { for } 0 \leq b \leq 1,
$$

We note that

$$
j_{\mathcal{K}, V}([S, M, n-p+1])=S\left(\begin{array}{cc}
\mathbf{m}_{1} & 0 \\
\vdots & \vdots \\
\mathbf{m}_{p-1} & 0 \\
\mathbf{0}_{p-1} & 1
\end{array}\right) \quad \text { for } b=0 .
$$

This map is well defined. In fact, suppose that $[S, T, M, n-p+1, b]=$ $\left[S^{\prime}, T^{\prime}, M^{\prime}, n-p+1, b\right]$ in $\mathcal{K}(n, p, n-p+1)$. Then we have $S^{\prime}=S\left({ }^{t} G \dot{+}(1)\right)$, $T^{\prime}=T_{2} T\left((1) \dot{+}{ }^{t} L\right)$ and $M^{\prime}=\left(G \dot{+} E_{n-p+1}\right)\left(E_{p} \dot{+} L\right) M$ by Remark 2.5. Hence, we have $j_{\mathcal{K}, V}([S, T, M, n-p+1, b])=j_{\mathcal{K}, V}\left(\left[S^{\prime}, T^{\prime}, M^{\prime}, n-p+1, b\right]\right)$. 
We show that $j_{\mathcal{K}, V}$ is a continuous injection. Suppose $j_{\mathcal{K}, V}([S, T, M, n-$ $p+1, b])=j_{\mathcal{K}, V}\left(\left[S^{\prime}, T^{\prime}, M^{\prime}, n-p+1, b\right]\right)$ for $b>0$. Then ${ }^{t} S S^{\prime} \mathbf{e}_{p}=\mathbf{e}_{p}$ and $S^{\prime}=S\left({ }^{t} G \dot{+}(1)\right)$. Since

$$
\left({ }^{t} G \dot{+}(1)\right)\left(\begin{array}{cc}
\mathbf{m}_{1} & 0 \\
\vdots & \vdots \\
\mathbf{m}_{p-1} & 0 \\
b \mathbf{m}_{p} & \sqrt{1-b^{2}}
\end{array}\right)=\left(\begin{array}{cc}
\mathbf{m}_{1}^{\prime} & 0 \\
\vdots & \vdots \\
\mathbf{m}_{p-1}^{\prime} & 0 \\
b \mathbf{m}_{p}^{\prime} & \sqrt{1-b^{2}}
\end{array}\right),
$$

we have $M^{\prime}=\left(G \dot{+} E_{n-p+1}\right)\left(E_{p} \dot{+} L\right) M$ for some $G \in O(p-1)$ and $L \in O(n-p)$. Furthermore, we have $T^{\prime}=T^{\prime}\left((1) \dot{+}^{t} L\right){ }^{t} T T\left((1) \dot{+}{ }^{t} L\right)$. The proof is similar for $b=0$.

Next we show that $j_{\mathcal{K}, V}$ is surjective. Let $M_{p \times(n+1)}$ be a $p \times(n+1)$-matrix in $V_{n+1, p}^{r o w}$. Then we have $S \in O(p)$ and $b \in[0,1]$ such that

$$
M_{p \times(n+1)}=S\left(\begin{array}{cc}
\mathbf{m}_{1} & 0 \\
\vdots & \vdots \\
\mathbf{m}_{p-1} & 0 \\
b \mathbf{m}_{p} & \sqrt{1-b^{2}}
\end{array}\right) .
$$

Indeed, if we write $M_{p \times(n+1)}=\left(\overline{\mathbf{u}}_{1}, \ldots, \overline{\mathbf{u}}_{n+1}\right)$ and $S=\left(\overline{\mathbf{s}}_{1}, \ldots, \overline{\mathbf{s}}_{p}\right)$, then we have $\overline{\mathbf{u}}_{n+1}=\sqrt{1-b^{2}} \overline{\mathbf{s}}_{p}$ and $b=\sqrt{1-\left\|\overline{\mathbf{u}}_{n+1}\right\|^{2}}$. Hence, $b$ is determined by $M_{p \times(n+1)}$. If $b<1$, then there exists an element $S \in O(p)$ such that $S\left(\sqrt{1-b^{2}} \mathbf{e}_{p}\right)=\overline{\mathbf{u}}_{n+1}$. Then we have

$$
{ }^{t} S M_{p \times(n+1)}=\left({ }^{t} S \overline{\mathbf{u}}_{1}, \ldots,{ }^{t} S \overline{\mathbf{u}}_{n}, \sqrt{1-b^{2}} \mathbf{e}_{p}\right),
$$

which lies in $V_{n+1, p}^{\text {row }}$. Let $M$ be any element of $O(n)$ such that $M\left(\begin{array}{l}1 \\ p\end{array}\right)=$ ${ }^{t} S\left(\overline{\mathbf{u}}_{1}, \ldots, \overline{\mathbf{u}}_{n}\right)$. Then we have

$$
j_{\mathcal{K}, V}\left(\left[S, E_{n-p+1}, M, n-p+1, b\right]\right)=M_{p \times(n+1)} .
$$

If $b=1$, then $\overline{\mathbf{u}}_{n+1}=\mathbf{0}$. Let $M$ be any element of $O(n)$ such that $M\left(\begin{array}{l}1 \\ p\end{array}\right)=$ $\left(\overline{\mathbf{u}}_{1}, \ldots, \overline{\mathbf{u}}_{n}\right)$. Then we have

$$
j_{\mathcal{K}, V}\left(\left[E_{p}, E_{n-p+1}, M, n-p+1,1\right]\right)=M_{p \times(n+1)} .
$$

Since both spaces $\mathcal{K}(n, p, n-p+1)$ and $V_{n+1, p}^{\text {row }}$ are compact, $j_{\mathcal{K}, V}$ is a homeomorphism. 
Let $(O, U) \in O(p) \times O(n)$. Then we have

$$
\begin{aligned}
j_{\mathcal{K}, V}((O, U) \cdot & {[S, T, M, n-p+1, b]) } \\
= & j_{\mathcal{K}, V}\left(\left[O S, T, M U^{-1}, n-p+1, b\right]\right) \\
= & O S\left(\begin{array}{cc}
\mathbf{m}_{1} U^{-1} & 0 \\
\vdots & \vdots \\
\mathbf{m}_{p-1} U^{-1} & 0 \\
b \mathbf{m}_{p} U^{-1} & \sqrt{1-b^{2}}
\end{array}\right) \\
= & O S\left(\begin{array}{cc}
\mathbf{m}_{1} & 0 \\
\vdots & \vdots \\
\mathbf{m}_{p-1} & 0 \\
b \mathbf{m}_{p} & \sqrt{1-b^{2}}
\end{array}\right)\left(U^{-1} \dot{+}(1)\right) \\
= & (O, U) \cdot j_{\mathcal{K}, V}([S, T, M, n-p+1, b]) .
\end{aligned}
$$

Hence, $j_{\mathcal{K}, V}$ is equivariant.

\section{$\S 3 . \quad$ Stable Homotopy Groups}

When $\sigma \neq 0$, we define

$$
\begin{aligned}
& r_{\sigma, n-p+1}: \mathcal{K}(n, p, \sigma, b) \rightarrow \mathcal{K}(n, p, n-p+1, b), \\
& r_{\sigma, n-p+1}^{\Sigma}: \Sigma \mathcal{K}(n, p, \sigma) \rightarrow \Sigma \mathcal{K}(n, p, n-p+1)
\end{aligned}
$$

to be the maps induced canonically from the inclusions $O(c) \times O(d) \rightarrow O(n-p$ $+1)$ respectively. Furthermore, we have the canonical retraction $r^{0}: \mathcal{K}(n, p, 0)$ $\backslash \Sigma \mathcal{K}(n, p, 0) \rightarrow V_{n, p}^{r o w}$. These maps canonically yield the retractions

$r_{\Omega, \mathcal{K}}: \Omega^{n-p+1,0}(n, p) \rightarrow \mathcal{K}(n, p, n-p+1), \quad$ when $n-p+1$ is odd, $r_{\Omega, \mathcal{K}}^{0}: \Omega^{n-p+1,0}(n, p) \backslash \Sigma \mathcal{K}(n, p, 0) \rightarrow \mathcal{K}(n, p, n-p+1)$, when $n-p+1$ is even,

which are equivariant with respect to the action of $O(p) \times O(n)$ satisfying that $R_{\Omega, \mathcal{K}} \circ j_{\mathcal{K}, V}$ is the identity of $\mathcal{K}(n, p, n-p+1)$.

We define a topological embedding

$$
i_{V, \Omega}: V_{n+1, p}^{r o w} \rightarrow \Omega^{n-p+1,0}(n, p)
$$

and

$$
\begin{array}{ll}
R_{\Omega, V}: \Omega^{n-p+1,0}(n, p) \rightarrow V_{n+1, p}^{\text {row }}, & \text { when } n-p+1 \text { is odd, } \\
R_{\Omega, V}^{0}: \Omega^{n-p+1,0}(n, p) \backslash \Sigma \mathcal{K}(n, p, 0) \rightarrow V_{n+1, p}^{\text {row }}, & \text { when } n-p+1 \text { is even }
\end{array}
$$


to be the compositions $i_{\mathcal{K}(n, p, n-p+1)} \circ j_{\mathcal{K}, V}^{-1}, j_{\mathcal{K}, V} \circ r_{\Omega, \mathcal{K}}$ and $j_{\mathcal{K}, V} \circ r_{\Omega, \mathcal{K}}^{0}$ respectively.

Let $\pi_{N}$ and $\pi_{P}$ be the projections of $N \times P$ onto $N$ and $P$ respectively. We set

$$
J^{2}(T N, T P)=\operatorname{Hom}\left(\pi_{N}^{*}(T N), \pi_{P}^{*}(T P)\right) \oplus \operatorname{Hom}\left(S^{2}\left(\pi_{N}^{*}(T N)\right), \pi_{P}^{*}(T P)\right)
$$

over $N \times P$, where $S^{2}\left(\pi_{N}^{*}(T N)\right)$ is the 2 -fold symmetric product of $\left(\pi_{N}^{*}(T N)\right)$. If we provide $N$ and $P$ with Riemannian metrics, then the Levi-Civita connection induces the exponential maps $\exp _{N}: T N \rightarrow N$ and $\exp _{P}: T P \rightarrow P([\mathrm{~K}-\mathrm{N}])$. We define a bundle map

$$
j_{\exp }: J^{2}(N, P) \rightarrow J^{2}(T N, T P) \quad \text { over } N \times P
$$

by sending $z=j_{x}^{2} f \in J_{x, y}^{2}(N, P)$ to the 2 -jet of $\left(\exp _{P} \mid T_{y} P\right)^{-1} \circ f \circ\left(\exp _{N} \mid T_{x} N\right)$ at $\mathbf{0} \in T_{x} N$, which is regarded as an element of $J^{2}\left(T_{x} N, T_{y} P\right)$. The structure group of $J^{2}(T N, T P)$ is reduced to $O(p) \times O(n)$. Set $J^{2}(n, p)=J_{0,0}^{2}\left(\mathbf{R}^{n}, \mathbf{R}^{p}\right)$ and $\Omega^{n-p+1,0}(n, p)=\Omega^{n-p+1,0}\left(\mathbf{R}^{n}, \mathbf{R}^{p}\right) \cap J^{2}(n, p)$. For a jet $z=j_{x}^{2} f \in$ $\Omega^{n-p+1,0}\left(\mathbf{R}^{n}, \mathbf{R}^{p}\right)$, we define $\pi_{\Omega}$ by $\pi_{\Omega}(z)=j_{0}^{2}(l(-f(x)) \circ f \circ l(x))$, where $l(a)$ denotes the parallel translation defined by $l(a)(x)=x+a$. In particular, we obtain a canonical diffeomorphism

$$
\pi_{\mathbf{R}^{n}}^{2} \times \pi_{\mathbf{R}^{p}}^{2} \times \pi_{\Omega}: \Omega^{n-p+1,0}\left(\mathbf{R}^{n}, \mathbf{R}^{p}\right) \rightarrow \mathbf{R}^{n} \times \mathbf{R}^{p} \times \Omega^{n-p+1,0}(n, p) .
$$

We note that $j_{\exp }\left(\Omega^{n-p+1,0}(N, P)\right)$ coincides with the subbundle of $J^{2}(T N$, $T P$ ) associated with $\Omega^{n-p+1,0}(n, p)$.

With the identification $V_{n+1, p}^{\text {row }}=\mathbf{V}\left(\mathbf{R}^{n+1}, \mathbf{R}^{p}\right)$, we have the fiber maps

$$
\begin{aligned}
i_{\mathbf{V}, \Omega}(N, P) & : \mathbf{V}\left(T N \oplus \theta_{N}, T P\right) \rightarrow \Omega^{n-p+1,0}(N, P), \\
R_{\Omega, \mathbf{V}}(N, P) & : \Omega^{n-p+1,0}(N, P) \rightarrow \mathbf{V}\left(T N \oplus \theta_{N}, T P\right), \\
R_{\Omega, \mathbf{V}}^{0}(N, P) & : \Omega^{w, 0}(N, P) \rightarrow \mathbf{V}\left(T N \oplus \theta_{N}, T P\right)
\end{aligned}
$$

associated to the maps $i_{V, \Omega}, R_{\Omega, V}$ and $R_{\Omega, V}^{0}$ respectively. Let $\Gamma\left(R_{\Omega, \mathbf{V}}\right)$ : $\Gamma(N, P) \rightarrow \Gamma\left(\mathbf{V}\left(T N \oplus \theta_{N}, T P\right)\right)$ be the map induced from the map $R_{\Omega, \mathbf{V}}(N$, $P)$ by $\left.\Gamma\left(R_{\Omega, \mathbf{V}}\right)(s)(x)=R_{\Omega, \mathbf{V}}(N, P)\right)(s(x))$ for $s \in \Gamma(N, P)$.

Proof of Theorems 0.1 and 0.2 . Since $R_{\Omega, V} \circ i_{V, \Omega}$ is the identity of $V_{n+1, p}^{r o w}$ $=\operatorname{Hom}\left(\mathbf{R}^{n+1}, \mathbf{R}^{p}\right)$, we have that $R_{\Omega, \mathbf{V}}(N, P) \circ i_{\mathbf{V}, \Omega}(N, P)$ is the identity of $\mathbf{V}\left(T N \oplus \theta_{N}, T P\right)$. This is the proof of Theorem 0.1 .

Next take any element $h \in \Gamma\left(\mathbf{V}\left(T N \oplus \theta_{N}, T P\right)\right)$. By [An4, Theorem 0.5], there exists a fold-map $f: N \rightarrow P$ such that $j^{2} f$ and $\Gamma\left(i_{V, \Omega}\right)(h)$ are homotopic as sections in $\Gamma(N, P)$. This is the proof of Theorem 0.2 . 
As for the results concerning Theorem 0.1 we refer to [E1, 3.8 and 3.9], [Sa, Lemma 3.1] and [An2, Theorem 1]. We must refer to [E1, 3.10] as a prior work concerning Theorem 0.2. A weaker assertion of Theorem 0.2 was proved in [An4, Theorem 0.1] without using the homotopy type of $\Omega^{n-p+1,0}(n, p)$.

Remark 3.1. When $n-p+1$ is even, we have that $R_{\Omega, \mathbf{V}}^{0}(N, P) \circ i_{\mathbf{V}, \Omega}(N$, $P)$ is the identity of $\mathbf{V}\left(T N \oplus \theta_{N}, T P\right)$.

Now we define the maps $\omega_{n, p}^{\mathfrak{N}}$ and $\omega_{n, p}^{\Omega}$ in Theorem 0.3. Let $\mathcal{G}$ refers to either $G_{n-p+1, \ell}$ or $\widetilde{G}_{n-p+1, \ell}$ and let $\omega_{n, p}$ refers to either $\omega_{n, p}^{\mathfrak{N}}$ or $\omega_{n, p}^{\Omega}$. Let $f: N \rightarrow P$ be a fold-map. Then $f$ determines an epimorphism $\Gamma\left(R_{\Omega, \mathbf{V}}\right)\left(j^{2} f\right)$ : $T N \oplus \theta_{N} \rightarrow T P$ covering $f$. Let $\xi$ be the kernel bundle of $\Gamma\left(R_{\Omega, \mathbf{V}}\right)\left(j^{2} f\right)$. Since $T N$ has the metric, we have the orthogonal projection $T N \oplus \theta_{N} \rightarrow \xi$ and the splitting $T N \oplus \theta_{N}=f^{*}(T P) \oplus \xi$. For the case $\Omega_{n}^{\text {fold }}$, $\xi$ has the canonical induced orientaion. Let $\widetilde{c_{\xi}}: \xi \rightarrow \gamma_{\mathcal{G}}^{n-p+1}$ be the bundle map covering a classifying map $c_{\xi}: N \rightarrow \mathcal{G}$. Then we have the natural bundle map

$$
b_{f}: T N \oplus \theta_{N}=f^{*}(T P) \oplus \xi \rightarrow T P \times \gamma_{\mathcal{G}}^{n-p+1} \quad \text { covering } f \times c_{\xi} .
$$

Let $\nu_{N}^{k+\ell}$ and $\nu_{P}^{k}$ be the normal bundles of embeddings, $N \rightarrow \mathbf{R}^{n+k+\ell}$ and $P \rightarrow \mathbf{R}^{n+k}$ with trivialization $t_{N}: T N \oplus \theta_{N} \oplus \nu_{N}^{k+\ell} \rightarrow \theta_{N}^{n+k+\ell+1}$ and $t_{P}:$ $T P \oplus \nu_{P}^{k} \rightarrow \theta_{P}^{n+k}$ respectively (see the details in [An3, Section 2]). We have the trivialization $t_{\mathcal{G}}: \gamma_{\mathcal{G}}^{n-p+1} \oplus \widehat{\gamma}_{\mathcal{G}}^{\ell} \rightarrow \theta_{\mathcal{G}}^{n-p+1+\ell}$. By using [An2, Proposition 3.3] for trivializations $t_{N}$ and

$$
\begin{aligned}
t_{P \times \mathcal{G}} & :\left(T P \times \gamma_{\mathcal{G}}^{n-p+1}\right) \oplus\left(\nu_{P}^{k} \times \hat{\gamma}_{\mathcal{G}}^{\ell}\right) \cong\left(T P \oplus \nu_{P}^{k}\right) \times\left(\gamma_{\mathcal{G}}^{n-p+1} \oplus \hat{\gamma}_{\mathcal{G}}^{\ell}\right) \\
& \stackrel{{ }^{t}{ }^{\times t_{\mathcal{G}}}}{\longrightarrow} \theta_{P \times \mathcal{G}}^{n+k+\ell+1}
\end{aligned}
$$

$b_{f}$ induces a bundle map

$$
\nu\left(b_{f}\right): \nu_{N}^{k+\ell} \rightarrow \nu_{P}^{k} \times \widehat{\gamma}_{\mathcal{G}}^{\ell} \quad \text { covering } f \times c_{\xi}
$$

determined up to homotopy such that $t_{P \times \mathcal{G}} \circ\left(b_{f} \oplus \nu\left(b_{f}\right)\right) \circ t_{N}^{-1}$ is homotopic to $\left(f \times c_{\xi}\right) \times i d_{\mathbf{R}^{n+k+\ell+1}}$. Let $\alpha_{N}: S^{n+k+\ell} \rightarrow T\left(\nu_{N}^{k+\ell}\right)$ be the Pontrjagin-Thom construction for the embedding of $N$ into $S^{n+k+\ell}$. Then $\omega_{n, p}(f)$ is defined to be the stable homotopy class of the composition $T\left(\nu\left(b_{f}\right)\right) \circ \alpha_{N}$, where $T\left(\nu_{P}^{k} \times \widehat{\gamma}_{\mathcal{G}}^{\ell}\right)$ is identified with $T\left(\nu_{P}^{k}\right) \wedge T\left(\widehat{\gamma}_{\mathcal{G}}^{\ell}\right)$.

We need to show that $\omega_{n, p}^{\mathfrak{N}}(f)$ and $\omega_{n, p}^{\Omega}(f)$ are well-defined.

Lemma 3.2. The maps $\omega_{n, p}^{\mathfrak{N}}(f)$ and $\omega_{n, p}^{\Omega}(f)$ are well-defined. Namely, they do not depend on the choices of an embedding of $N$, of a representative $f$ of 
the fold-cobordism class $[f] \in \mathfrak{N}_{\text {fold }}^{n}(P)$ or $\Omega_{\text {fold }}^{n}(P)$, and Riemannian metrics of $N$ and $P$.

Proof. We first prove that $\omega_{n, p}$ does not depend on the choice of an embedding of $N$. Let $e_{N}^{\prime}: N \rightarrow \mathbf{R}^{n+k+\ell}$ be another embedding with normal bundles $\nu_{N}^{\prime}$, the trivialization $t_{N}^{\prime}: T_{N} \oplus \theta_{N} \oplus \nu_{N}^{\prime} \rightarrow \theta_{N}^{n+k+\ell+1}$ and a bundle map $\nu\left(b_{f}\right)^{\prime}: \nu_{N}^{\prime} \rightarrow \nu_{P}^{k} \times \widehat{\gamma}_{\mathcal{G}}^{\ell}$. Let $\alpha_{N}^{\prime}$ be the corresponding Pontryagin-Thom construction. Then by [An3, Remark 2.2] there exists a bundle map $b_{N}: \nu_{N} \rightarrow$ $\nu_{N}^{\prime}$. They yields $\nu\left(b_{f}\right) \circ b_{N}^{-1} \simeq \nu\left(b_{f}\right)^{\prime}: \nu_{N}^{\prime} \rightarrow \nu_{P}^{k} \times \widehat{\gamma}_{\mathcal{G}}^{\ell}$. Then we have

$$
\begin{aligned}
{\left[T\left(\nu\left(b_{f}\right)^{\prime}\right) \circ \alpha_{N}^{\prime}\right] } & =\left[T\left(\nu\left(b_{f}\right)\right) \circ T\left(b_{N}^{-1}\right) \circ T\left(b_{N}\right) \circ \alpha_{N}\right] \\
& =\left[T\left(\nu\left(b_{f}\right)\right) \circ \alpha_{N}\right] .
\end{aligned}
$$

Next we prove that $\omega_{n, p}$ does not depend on the choice of a representative $f$ of the fold-cobordism class $[f]$. Let $f_{i}: N_{i} \rightarrow P(i=0,1)$ be two foldmaps, where $N_{i}$ are closed (resp. oriented) smooth manifolds with a (resp. an oriented-) fold-cobordism $F:(W, \partial W) \rightarrow(P \times[0,1], P \times 0 \cup P \times 1)$ as in Introduction such that $F \mid N_{0}=f_{0}$ and $F \mid N_{1}=f_{1}$, for which we have the followings constructed similarly as for the fold-map $f$ :

(i) epimorphisms $\Gamma\left(R_{\Omega, \mathbf{V}}\right)\left(j^{2} f_{i}\right): T N_{i} \oplus \theta_{N_{i}} \rightarrow T P$ covering $f_{i}$,

(ii) the kernel bundle $\xi_{i}$ of $\Gamma\left(R_{\Omega, \mathbf{v}}\right)\left(j^{2} f_{i}\right)$,

(iii) the orthogonal projection $T N_{i} \oplus \theta_{N_{i}} \rightarrow \xi_{i}$, the splitting $T N_{i} \oplus \theta_{N_{i}}=$ $f^{*}(T P) \oplus \xi_{i}$, and the canonical induced orientaion of $\xi_{i}$, when $\mathcal{G}$ is $\widetilde{G}_{n-p+1, \ell}$,

(iv) the bundle map $\widetilde{c_{\xi_{i}}}: \xi_{i} \rightarrow \gamma_{\mathcal{G}}^{n-p+1}$ covering a classifying map $c_{\xi_{i}}: N_{i} \rightarrow \mathcal{G}$,

(v) the natural bundle map $b_{f}: T N_{i} \oplus \theta_{N_{i}}=f^{*}(T P) \oplus \xi_{i} \rightarrow T P \times \gamma_{\mathcal{G}}^{n-p+1}$ covering $f_{i} \times c_{\xi_{i}}$,

(vi) the normal bundle $\nu_{N_{i}}^{k+\ell}$ of embeddings, $N_{i} \rightarrow \mathbf{R}^{n+k+\ell}$ with trivializations $t_{N_{i}}: T N_{i} \oplus \theta_{N_{i}} \oplus \nu_{N_{i}}^{k+\ell} \rightarrow \theta_{N_{i}}^{n+k+\ell+1}$,

(vii) bundle maps $\nu\left(b_{f_{i}}\right): \nu_{N_{i}}^{k+\ell} \rightarrow \nu_{P}^{k} \times \widehat{\gamma}_{\mathcal{G}}^{\ell}$ covering $f_{i} \times c_{\xi_{i}}$ determined up to homotopy such that $t_{P \times \mathcal{G}} \circ\left(b_{f_{i}} \oplus \nu\left(b_{f_{i}}\right)\right) \circ t_{N_{i}}^{-1}$ is homotopic to $\left(f_{i} \times c_{\xi_{i}}\right) \times$ $i d_{\mathbf{R}^{n+k+\ell+1}}$,

(viii) the Pontrjagin-Thom construction $\alpha_{N_{i}}: S^{n+k+\ell} \rightarrow T\left(\nu_{N_{i}}^{k+\ell}\right)$ for the embedding of $N_{i}$ into $\mathbf{R}^{n+k+\ell}$,

(ix) the homotopy classes $\omega_{n, p}\left(f_{i}\right)$ of the composition $T\left(\nu\left(b_{f_{i}}\right)\right) \circ \alpha_{N_{i}}$. 
By Theorem 0.1, the fold map $F$ determines an epimorphism $\Gamma\left(R_{\Omega, \mathbf{V}}\right)$ $\left(j^{2} F\right): T W \oplus \theta_{W} \rightarrow T(P \times I)$ covering $F$. Let $\xi_{F}$ be the kernel bundle of $\Gamma\left(R_{\Omega, \mathbf{V}}\right)\left(j^{2} F\right)$ such that $\left.\xi_{F}\right|_{N \times i}=\xi_{i}$. Since $T W$ has the metric compatible with that of $T N_{i} \oplus \theta_{N_{i}}$, we have the orthogonal projection $T W \oplus \theta_{W} \rightarrow \xi_{F}$ and the splitting $T W \oplus \theta_{W}=f^{*}(T(P \times I)) \oplus \xi_{F}$. Therefore, $\xi_{F}$ has the canonical induced orientation when $\mathcal{G}$ is $\widetilde{G}_{n-p+1, \ell}$. Let $\widetilde{c_{\xi_{F}}}: \xi_{F} \rightarrow \gamma_{\mathcal{G}}^{n-p+1}$ be the bundle map covering a classifying map $c_{\xi_{F}}: W \rightarrow \mathcal{G}$. Hence, we have the natural bundle map $b_{F}: T W \oplus \theta_{W}=f^{*}(T(P \times I)) \oplus \xi_{F} \rightarrow T(P \times I) \times \gamma_{\mathcal{G}}^{n-p+1}$ covering $F \times c_{\xi_{F}}$. Let $\nu_{W}^{k+\ell}$ and $\nu_{P \times I}^{k}$ be the normal bundles of embeddings, $W \rightarrow \mathbf{R}^{n+k+\ell} \times I$ and $P \times I \rightarrow \mathbf{R}^{n+k} \times I$ with trivialization $t_{W}: T W \oplus \theta_{W}$ $\oplus \nu_{W}^{k+\ell} \rightarrow \theta_{W}^{n+k+\ell+1}$ and $t_{P \times I}: T(P \times I) \oplus \nu_{P \times I}^{k} \rightarrow \theta_{P \times I}^{n+k+1}$ respectively. By using [An2, Proposition 3.3] for trivializations $t_{W}$ and

$$
\begin{aligned}
t_{(P \times I) \times \mathcal{G}}:\left(T(P \times I) \times \gamma_{\mathcal{G}}^{n-p+1}\right) \oplus\left(\nu_{P \times I}^{k} \times \hat{\gamma}_{\mathcal{G}}^{\ell}\right) \\
\cong\left(T(P \times I) \oplus \nu_{P \times I}^{k}\right) \times\left(\gamma_{\mathcal{G}}^{n-p+1} \oplus \widehat{\gamma}_{\mathcal{G}}^{\ell}\right) \stackrel{t_{P \times I} \times t_{\mathcal{G}}}{\longrightarrow} \theta_{(P \times I) \times \mathcal{G}}^{n+k+\ell+2},
\end{aligned}
$$

$b_{F}$ induces a bundle map $\nu\left(b_{F}\right): \nu_{W}^{k+\ell} \rightarrow \nu_{P \times I}^{k} \times \widehat{\gamma}_{\mathcal{G}}^{\ell}$ covering $F \times c_{\xi_{F}}$ determined up to homotopy. Let $\alpha_{W}: S^{n+k+\ell} \times I \rightarrow T\left(\nu_{W}^{k+\ell}\right)$ be the Pontrjagin-Thom construction for the embedding of $W$ into $\mathbf{R}^{n+k+\ell} \times I$. Let $\omega_{n, p}(F)$ be the composition $T\left(\nu\left(b_{W}\right)\right) \circ \alpha_{W}$. If we restrict these constructions for $W$ to $N_{i}$ and $P \times i$, then we obtain the properties observed in (i)-(ix) above. Hence, $\omega_{n, p}(W)$ gives a homotopy of $\omega_{n, p}\left(f_{0}\right)$ and $\omega_{n, p}\left(f_{1}\right)$.

We show that $\omega_{n, p}(f)$ does not depend on the choices of Riemannian metrics of $N$ and $P$. This follows from the fact that Riemannian metrics are all homotopic (see [Ste, 12.12]).

Proof of Theorem 0.3. We give a proof only for the case $\Omega_{\text {fold }}^{n}(P)$, since the proof for the case $\mathfrak{N}_{\text {fold }}^{n}(P)$ is analougous.

We prove the surjectivity of $\omega_{n, p}^{\Omega}$. Let $\alpha: S^{n+k+\ell} \rightarrow T\left(\nu_{P}^{k} \times \widehat{\gamma}_{\bar{G}_{n-p+1, \ell}^{\ell}}^{\ell}\right)=$ $T\left(\nu_{P}^{k}\right) \wedge T\left(\widehat{\gamma}_{\widetilde{G}_{n-p+1, \ell}^{\ell}}^{\ell}\right)$. We may assume that $\alpha$ is transverse to the zero-section $P \times \widetilde{G}_{n-p+1, \ell}$. Set $N=\alpha^{-1}\left(P \times \widetilde{G}_{n-p+1, \ell}\right)$ with normal bundle $\nu_{N}^{k+\ell}$ and $c_{N}=\alpha \mid N$. Then there exists a bundle map

$$
h_{\nu_{N}}: \nu_{N}^{k+\ell} \rightarrow \nu_{P}^{k} \times \widehat{\gamma}_{\widetilde{G}_{n-p+1, \ell}^{\ell}}^{\ell} \quad \text { covering } c_{N},
$$

which, by [An2, Proposition 3.3], induces a bundle map

$$
\begin{aligned}
h_{\tau_{N}} & : T N \oplus \theta_{N}^{k^{\prime}+k^{\prime \prime}+1} \rightarrow\left(T P \oplus \theta_{P}^{k^{\prime}}\right) \times\left(\gamma_{\widetilde{G}_{n-p+1, \ell}}^{n-p+1} \oplus \theta_{\widetilde{G}_{n-p+1, \ell}^{\prime \prime}}^{k^{\prime \prime}}\right) \\
& =\left(T P \times \gamma_{\widetilde{G}_{n-p+1, \ell}}^{n-p+1}\right) \oplus \theta_{P \times \widetilde{G}_{n-p+1, \ell}}^{k^{\prime}+k^{\prime \prime}} \quad \text { covering } c_{N}
\end{aligned}
$$


such that $\left(t_{P \times \widetilde{G}_{n-p+1, \ell}} \oplus i d_{\theta_{P \times \widetilde{G}_{n-p+1, \ell}}^{k^{\prime}+k^{\prime \prime}}}\right) \circ\left(h_{\tau_{N}} \oplus h_{\nu_{N}}\right) \circ\left(t_{N} \oplus i d_{\theta_{N}^{k^{\prime}+k^{\prime \prime}}}\right)^{-1}$ is homotopic to $c_{N} \times i d_{\mathbf{R}^{n+k+\ell+k^{\prime}+k^{\prime \prime}+1}}$. Let $p_{P}: P \times \widetilde{G}_{n-p+1, \ell} \rightarrow P$ and $p_{\widetilde{G}_{n-p+1, \ell}}: P \times \widetilde{G}_{n-p+1, \ell} \rightarrow \widetilde{G}_{n-p+1, \ell}$ be canonical projections respectively. By the dimensional reason considering $T N \oplus \theta_{N}^{k^{\prime}+k^{\prime \prime}+1}$ and $\left(p_{P} \circ c_{N}\right)^{*}(T P) \oplus$ $\left(p_{\widetilde{G}_{n-p+1, \ell}} \circ c_{N}\right)^{*}\left(\gamma_{\widetilde{G}_{n-p+1, \ell}-p+1}^{n-p}\right) \oplus \theta_{N}^{k^{\prime}+k^{\prime \prime}+1}$, there exists a bundle map

$$
\widetilde{h}: T N \oplus \theta_{N} \rightarrow T P \times \gamma_{\widetilde{G}_{n-p+1, \ell}}^{n-p+1} \quad \text { covering } \quad c_{N},
$$

such that $\widetilde{h} \times i d_{\mathbf{R}^{k^{\prime}+k^{\prime \prime}}}$ is homotopic to $h_{\tau_{N}}$. Let $p_{T P}: T P \times \gamma_{\widetilde{G}_{n-p+1, \ell}}^{n-p+1} \rightarrow T P$ be the canonical projection. Then it follows from Theorem 0.2 that $p_{T P} \circ \tilde{h}$ : $T N \oplus \theta_{N} \rightarrow T P$ has a fold-map $f: N \rightarrow P$ such that $\Gamma\left(R_{\Omega, \mathbf{V}}\right)\left(j^{2} f\right)$ is homotopic to $p_{T P} \circ \widetilde{h}$ in $\Gamma\left(\mathbf{V}\left(T N \oplus \theta_{N}, T P\right)\right)$. Hence, $b_{f}$ is homotopic to $\widetilde{h}$. This shows that $\nu\left(b_{f}\right)$ is homotopic to $h_{\nu_{N}}$. By the definition of $\omega_{n, p}^{\Omega}$, we have that

$$
\omega_{n, p}^{\Omega}(f)=\left[T\left(\nu\left(b_{f}\right)\right) \circ \alpha_{N}\right]=\left[T\left(h_{\nu_{N}}\right) \circ \alpha_{N}\right]=\alpha .
$$

This completes the proof.

Remark 3.3. In this remark a smooth map $f: N \rightarrow P$ is called $a$ quasidefinite fold-map if $f$ has only fold singularities of non-zero signatures. Let $\mathfrak{N}_{n}^{q . d . f o l d}(P)$ (resp. $\Omega_{n}^{q . d . f o l d}(P)$ ) denote the set consisiting of all quasidefinite (resp. oriented-) fold-cobordism classes of quasidefinite fold-maps into $P$, which are defined analogously as $\mathfrak{N}_{n}^{\text {fold }}(P)$ (resp. $\Omega_{n}^{\text {fold }}(P)$ ) in Introduction by replacing fold-maps with quasidefinite fold-maps. When $n-p+1$ is odd, a quasidefinite fold-map coincides with a fold-map, and hence we have $\mathfrak{N}_{n}^{q . d . f o l d}(P)=\mathfrak{N}_{n}^{\text {fold }}(P)$ (resp. $\Omega_{n}^{\text {q.d.fold }}(P)=\Omega_{n}^{\text {fold }}(P)$ ). When $n-p+1$ is even, we can define the maps

$$
\begin{aligned}
& \bar{\omega}_{n, p}^{\mathfrak{N}}: \mathfrak{N}_{n}^{q . d . f o l d}(P) \rightarrow \lim _{k \rightarrow \infty} \pi_{n+k+\ell}\left(T\left(\nu_{P}^{k}\right) \wedge T\left(\widehat{\gamma}_{G_{n-p+1, \ell}}^{\ell}\right)\right), \\
& \bar{\omega}_{n, p}^{\Omega}: \Omega_{n}^{q . d . f o l d}(P) \rightarrow \lim _{k \rightarrow \infty} \pi_{n+k+\ell}\left(T\left(\nu_{P}^{k}\right) \wedge T\left(\widehat{\gamma}_{\widetilde{G}_{n-p+1, \ell}^{\ell}}\right)\right)
\end{aligned}
$$

similarly as in the case of $\mathfrak{N}_{n}^{\text {fold }}(P)$ (resp. $\Omega_{n}^{\text {fold }}(P)$ ). However, we cannot assert that $\bar{\omega}_{n, p}^{\mathfrak{N}}$ and $\bar{\omega}_{n, p}^{\Omega}$ are surjective, because the homotopy principle does not hold for quasidefinite fold-maps (see [An4, Theorem 0.5]).

Let $\mathbf{f}: N \rightarrow P$ be a submersion. We study the element $\bar{\omega}_{n, p}(\mathbf{f})$, where $\bar{\omega}_{n, p}$ refers to either $\bar{\omega}_{n, p}^{\mathfrak{N}}$ or $\bar{\omega}_{n, p}^{\Omega}$. Let $\mathfrak{G}$ denote either $G_{n-p, \ell}$ or $\widetilde{G}_{n-p, \ell}$ depending on whether $\mathcal{G}$ is either $G_{n-p+1, \ell}$ or $\widetilde{G}_{n-p+1, \ell}$. Let $i_{\mathfrak{G}, \mathcal{G}}: \mathfrak{G} \rightarrow \mathcal{G}$ be the inclusion induced from the inclusion $\mathbf{R}^{n-p+\ell}=\mathbf{R}^{n-p+\ell} \times 0 \subset \mathbf{R}^{n-p+\ell+1}$. Then the 
classifying bundle maps $\widetilde{i_{\mathfrak{G}, \mathcal{G}}}: \gamma_{\mathfrak{G}}^{n-p} \oplus \theta_{\mathfrak{G}} \rightarrow \gamma_{\mathcal{G}}^{n-p+1}$ and the canonical bundle map $\widehat{i_{\mathfrak{G}, \mathcal{G}}}: \widehat{\gamma}_{\mathfrak{G}}^{\ell} \rightarrow \widehat{\gamma}_{\mathcal{G}}^{\ell}$ covering $i_{\mathfrak{G}, \mathcal{G}}$. They induce

$$
\begin{aligned}
& T\left(\widetilde{i_{\mathfrak{G}, \mathcal{G}}}\right): T\left(\gamma_{\mathfrak{G}}^{n-p} \oplus \theta_{\mathfrak{G}}\right)=S\left(T\left(\gamma_{\mathfrak{G}}^{n-p}\right)\right) \rightarrow T\left(\gamma_{\mathcal{G}}^{n-p+1}\right), \\
& T\left(\widehat{i_{\mathfrak{G}, \mathcal{G}}}\right): T\left(\widehat{\gamma}_{\mathfrak{G}}^{\ell}\right) \rightarrow T\left(\widehat{\gamma}_{\mathcal{G}}^{\ell}\right)
\end{aligned}
$$

respectively. Let

$$
\mathbf{j}_{\mathfrak{G}, \mathcal{G}}: \lim _{k \rightarrow \infty} \pi_{n+k+\ell}\left(T\left(\nu_{P}^{k}\right) \wedge T\left(\widehat{\gamma}_{\mathfrak{G}}^{\ell}\right)\right) \rightarrow \lim _{k \rightarrow \infty} \pi_{n+k+\ell}\left(T\left(\nu_{P}^{k}\right) \wedge T\left(\widehat{\gamma}_{\mathcal{G}}^{\ell}\right)\right)
$$

be the map defined by sending $c$ to $\left(i d_{T\left(\nu_{P}^{k}\right)} \wedge T\left(\widehat{i_{\mathfrak{G}, \mathcal{G}}}\right)\right)_{*}(c)$. In the following proposition let $L$ be a closed (resp. oriented) manifold of dimension $n-p$, which is embedded in $\mathbf{R}^{n-p+\ell}$. Let $\alpha_{L}: S^{n-p+\ell} \rightarrow T\left(\nu_{L}^{\ell}\right)$ be the Pontrjagin-Thom construction and let $\widetilde{{\nu_{L}^{\ell}}^{\ell}}: \nu_{L}^{\ell} \rightarrow \widehat{\gamma}_{\mathfrak{G}}^{\ell}$ be the bundle map covering a claasifying $\operatorname{map} c_{\nu_{L}^{\ell}}: L \rightarrow \mathfrak{G}$.

Proposition 3.4. Let $\ell \gg n$. (1) Let $\mathbf{f}: N \rightarrow P$ be a submersion. Then $\bar{\omega}_{n, p}(\mathbf{f})$ lies in the image of $\mathbf{j}_{\mathfrak{G}, \mathcal{G}}$, where $\bar{\omega}_{n, p}$ refers to either $\bar{\omega}_{n, p}^{\Omega}$ or $\bar{\omega}_{n, p}^{\mathfrak{N}}$ depending on whether $N$ and $P$ are provided with orientations or not.

(2) Let $L$ be a manifold as above and let $p_{P}: L \times P \rightarrow P$ be the canonical projection. Then $\bar{\omega}_{n, p}\left(p_{P}\right)$ is the stable homotopy class of $\alpha_{P} \wedge\left(T\left(\widehat{i_{\mathfrak{G}, \mathcal{G}}}\right) \circ\right.$ $\left.T\left(\widetilde{{\nu_{L}^{\ell}}^{\prime}}\right) \circ \alpha_{L}\right)$.

Proof. Let $\xi^{\prime}$ be the kernel bundle $\operatorname{Ker}(d \mathbf{f})$ over $N$, which is the subbundle of $T N$ along the fibers of $\mathbf{f}$. Let $\widetilde{c_{\xi^{\prime}}}: \xi^{\prime} \rightarrow \gamma_{\mathfrak{G}}^{n-p}$ be the bundle map covering the classifying map $c_{\xi^{\prime}}: N \rightarrow \mathfrak{G}$ and $\pi_{\xi^{\prime}}: T N \rightarrow \xi^{\prime}$ be the orthogonal projection. Then we have a bundle map

$$
b_{\mathbf{f}}^{\prime}=d \mathbf{f} \times\left(\widetilde{c_{\xi^{\prime}}} \circ \pi_{\xi^{\prime}}\right): T N \rightarrow T P \times \gamma_{\mathfrak{G}}^{n-p} .
$$

Let

$$
\begin{aligned}
t_{T N \oplus \nu}^{\prime} & : T N \oplus \nu_{N}^{k+\ell} \rightarrow \theta_{N}^{n+k+\ell}, \\
t_{P \times \mathfrak{G}} & :\left(T P \times \gamma_{\mathfrak{G}}^{n-p}\right) \oplus\left(\nu_{P}^{k} \times \widehat{\gamma}_{\mathfrak{G}}^{\ell}\right) \cong\left(T P \oplus \nu_{P}^{k}\right) \times\left(\gamma_{\mathfrak{G}}^{n-p} \oplus \widehat{\gamma}_{\mathfrak{G}}^{\ell}\right) \stackrel{t_{P} \times t_{\mathfrak{G}}}{\longrightarrow} \theta_{P \times \mathfrak{G}}^{n+k+\ell},
\end{aligned}
$$

be trivializations defined similarly as in (3.5). By [An2, Propositiion 3.3] $b_{\mathbf{f}}^{\prime}$ induces a bundle map $\nu\left(b_{\mathbf{f}}^{\prime}\right): \nu_{N}^{k+\ell} \rightarrow \nu_{P}^{k} \times \widehat{\gamma}_{\mathfrak{G}}^{\ell}$ such that $t_{P \times \mathfrak{G}} \circ\left(b_{\mathbf{f}}^{\prime} \oplus\right.$ $\left.\nu\left(b_{\mathbf{f}}^{\prime}\right)\right) \circ\left(t_{T N \oplus \nu}^{\prime}\right)^{-1}$ is homotopic to $\left(\mathbf{f} \times c_{\xi^{\prime}}\right) \times i d_{\mathbf{R}^{n+k+\ell}}$. By the definition of $\Gamma\left(R_{\Omega, \mathbf{V}}\right)\left(j^{2} \mathbf{f}\right)$, we know that $\Gamma\left(R_{\Omega, \mathbf{V}}\right)\left(j^{2} \mathbf{f}\right)$ is homotopic to $d \mathbf{f} \circ p_{T N}: T N \oplus$ $\theta_{N} \rightarrow T N \rightarrow T P$, where $p_{T N}$ is the canonical projection $T N \oplus \theta_{N} \rightarrow T N$. Since $\xi_{\mathbf{f}}=\xi^{\prime} \oplus \theta_{N}$, we may set

$$
b_{\mathbf{f}}=\left(i d_{T P} \times \widetilde{i_{\mathfrak{G}, \mathcal{G}}}\right) \circ \overline{b_{\mathbf{f}}^{\prime}}: T N \oplus \theta_{N} \rightarrow T P \times\left(\gamma_{\mathfrak{G}}^{n-p} \oplus \theta_{\mathfrak{G}}\right) \rightarrow T P \times \gamma_{\mathcal{G}}^{n-p+1},
$$


where $\overline{b_{\mathbf{f}}^{\prime}}(\mathbf{v}, t)=\left(b_{\mathbf{f}}^{\prime}(\mathbf{v}), t\right)$. Hence, we may set

$$
\nu\left(b_{\mathbf{f}}\right)=\left(i d_{\nu_{P}^{k}} \times \widehat{i_{\mathfrak{G}, \mathcal{G}}}\right) \circ \nu\left(b_{\mathbf{f}}^{\prime}\right): \nu_{N}^{k+\ell} \rightarrow \nu_{P}^{k} \times \widehat{\gamma}_{\mathfrak{G}}^{\ell} \rightarrow \nu_{P}^{k} \times \widehat{\gamma}_{\mathcal{G}}^{\ell} .
$$

Therefore, $\bar{\omega}_{n, p}(\mathbf{f})$ is the stable homotopy class of $T\left(i d_{\nu_{P}^{k}} \times \widehat{i_{\mathfrak{G}, \mathcal{G}}}\right) \circ T\left(\nu\left(b_{\mathbf{f}}^{\prime}\right)\right)$ $\circ \alpha_{N}$. This proves the assertion (1).

The differential $d p_{P}\left(=b_{p_{L}}^{\prime}\right): T L \times T P \rightarrow T P$ is the canonical projection and $\xi^{\prime}=p_{L}{ }^{*}(T L)$ for the canonical projection $p_{L}: L \times P \rightarrow L$. We have

$$
\nu\left(d p_{P}\right)=i d_{\nu_{P}^{k}} \times \widetilde{c_{\nu_{L}^{\ell}}}: \nu_{L \times P}=\nu_{P}^{k} \times \nu_{L}^{\ell} \rightarrow \nu_{P}^{k} \times \widehat{\gamma}_{\mathfrak{G}}^{\ell} .
$$

This yields

$$
\nu\left(b_{p_{L}}\right)=\left(i d_{\nu_{P}^{k}} \times \widehat{i_{\mathfrak{G}, \mathcal{G}}}\right) \circ \nu\left(d p_{P}\right): \nu_{P}^{k} \times \nu_{L}^{\ell} \rightarrow \nu_{P}^{k} \times \widehat{\gamma}_{\mathcal{G}}^{\ell} .
$$

By definition, we obtain that $\bar{\omega}_{n, p}\left(p_{P}\right)$ is the stable homotopy class of

$$
\begin{aligned}
& T\left(i d_{\nu_{P}^{k}} \times \widehat{i_{\mathfrak{G}, \mathcal{G}}}\right) \circ T\left(\nu\left(d p_{P}\right)\right) \circ \alpha_{L \times P} \\
& =\left(T\left(i d_{\nu_{P}^{k}}\right) \wedge T\left(\widehat{i_{\mathfrak{G}, \mathcal{G}}}\right)\right) \circ\left(T\left(i d_{\nu_{P}^{k}}\right) \wedge T\left(\widetilde{{\nu_{L}^{\ell}}_{L}}\right)\right) \circ\left(\alpha_{P} \wedge \alpha_{L}\right) \\
& =\alpha_{P} \wedge\left(T\left(\widehat{i_{\mathfrak{G}, \mathcal{G}}}\right) \circ T\left(\widetilde{{\nu_{L}^{\ell}}_{L}}\right) \circ \alpha_{L}\right) .
\end{aligned}
$$

This proves the assertion (2).

Let $W_{i}$ and $P_{i}$ be the $i$-th Stiefel-Whitney class and the $i$-th Pontrjagin class respectively. Let $I=\left(i_{1}, \ldots, i_{t}\right), J=\left(j_{1}, \ldots, j_{u}\right), W_{I}(\zeta)=W_{i_{1}}(\zeta) \cdots$ $W_{i_{t}}(\zeta), P_{J}(\zeta)=P_{j_{1}}(\zeta) \cdots P_{j_{u}}(\zeta)$ and so on. The following proposition is proved by a routine argument about characteristic classes (see $[\mathrm{H}])$.

Proposition 3.5. $\quad$ Let $N$ and $P$ be closed manifolds of dimensions $n$ and $p$ respectively. Let $f: N \rightarrow P$ be a quasidefinite fold-map (resp. submersion).

(1) Let $i_{1}+\cdots+i_{t}+j_{1}+\cdots+j_{u}=n$. Then the Stiefel-Whitney num$\operatorname{ber}\left(W_{I}\left(f^{*}(T P)\right) W_{J}\left(T N-f^{*}(T P)\right),[N]\right)$ is a quasidefintite fold-cobordism invariant. Unless $i_{1}+\cdots+i_{t} \leq p$ and $j_{1}, \ldots, j_{u} \leq n-p+1$ (resp. $\left.j_{1}, \ldots, j_{u} \leq n-p\right)$, then $\left(W_{I}\left(f^{*}(T P)\right) W_{J}\left(T N-f^{*}(T P)\right),[N]\right)$ vanishes.

(2) Let $N$ and $P$ be oriented and $4\left(i_{1}+\cdots+i_{t}+j_{1}+\cdots+j_{u}\right)=n$. Then the Pontrjagin number $\left(P_{I}\left(f^{*}(T P)\right) P_{J}\left(T N-f^{*}(T P)\right),[N]\right)$ is a quasidefintite oriented-fold-cobordism invariant. Unless $4\left(i_{1}+\cdots+i_{t}\right) \leq p$ and $4 j_{1}, \ldots, 4 j_{u} \leq n-p+1$ (resp. $\left.4 j_{1}, \ldots, 4 j_{u} \leq n-p\right)$, then $\left(P_{I}\left(f^{*}(T P)\right) P_{J}\right.$ $\left.\left(T N-f^{*}(T P)\right),[N]\right)$ vanishes. 
We now prove Theorem 0.4 , which is a special case of a result in [An5].

Proof of Theroem 0.4. Let $G$ refer to $G_{n, \ell}$ or $\widetilde{G}_{n, \ell}$. We provide $N$ and $P$ with Riemannian metrics. In the proof we always identify $J^{2}(N, P)$ and $\Omega^{n-p+1,0}(N, P)$ with $J^{2}(T N, T P)$ and $\Omega^{n-p+1,0}(T N, T P)$ respectively by (3.2). Let $f: N \rightarrow P$ be a fold-map. Let $B_{T N}: T N \rightarrow \gamma_{G}^{n}$ be a bundle map covering a classifying map $c_{N}: N \rightarrow G$. Then $B_{T N}$ induces bundle maps $B_{J}$ : $J^{2}(T N, T P) \rightarrow J^{2}\left(\gamma_{G}^{n}, T P\right)$ and $B_{\Omega}: \Omega^{n-p+1,0}(T N, T P) \rightarrow \Omega^{n-p+1,0}\left(\gamma_{G}^{n}, T P\right)$ covering $c_{N} \times i d_{P}$. It is easy to see that $p_{G} \circ B_{\Omega} \circ j^{2} f=c_{N}$ and $p_{P} \circ B_{\Omega} \circ j^{2} f$ $=f$. We have the commutative diagram

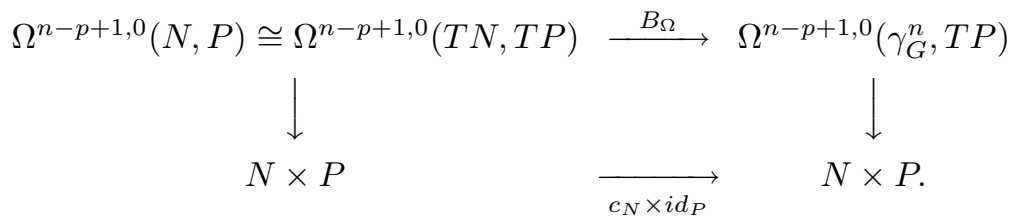

We have the trivializations $t_{N}: T N \oplus \nu_{N}^{\ell} \rightarrow \theta_{N}^{n+\ell}$ and $t_{G}: \gamma_{G}^{n} \oplus \widehat{\gamma}_{G}^{\ell} \rightarrow \theta_{G}^{n+\ell}$. Here, we should recall the definition of the bundel maps $B_{T N}: T N \rightarrow \gamma_{G}^{n}$ and $B_{\nu_{N}}: \nu_{N}^{\ell} \rightarrow \widehat{\gamma}_{G}^{\ell}$. For a point $x \in \mathbf{R}^{n+\ell}$, let $\ell_{x}: T_{x} \mathbf{R}^{n+\ell} \rightarrow \mathbf{R}^{n+\ell}$ be the canonical isomorphism. Then $B_{T N}$ maps $(x, \mathbf{v}) \in T_{x} N$ to $\left(\ell_{x}\left(T_{x} N\right), \ell_{x}(\mathbf{v})\right) \in$ $\gamma_{G}^{n}$, and $B_{\nu_{N}}$ maps $(x, \mathbf{w}) \in \nu_{N}^{\ell}$ to $\left(\ell_{x}\left(\left(\nu_{N}^{\ell}\right)_{x}\right), \ell_{x}(\mathbf{w})\right) \in \widehat{\gamma}_{G}^{\ell}$. Let $B_{p_{G}^{*}\left(\gamma_{G}^{n}\right)}$ : $p_{G}^{*}\left(\gamma_{G}^{n}\right) \rightarrow \gamma_{G}^{n}$ and $B_{p_{G}^{*}\left(\hat{\gamma}_{G}^{\ell}\right)}: p_{G}^{*}\left(\widehat{\gamma}_{G}^{\ell}\right) \rightarrow \widehat{\gamma}_{G}^{\ell}$ be the canonical bundle maps induced from $p_{G}$. Since $p_{G} \circ B_{\Omega} \circ J_{\exp } \circ j^{2} f=c_{N}, B_{T N}$ and $c_{N}$ induce bundle maps

$B_{T N}^{\Omega}:\left.T N \rightarrow p_{G}^{*}\left(\gamma_{G}^{n}\right)\right|_{\Omega^{n-p+1,0}\left(\gamma_{G}^{n}, P\right)} \quad$ and $\quad B_{\nu_{N}}^{\Omega}:\left.\nu_{N}^{\ell} \rightarrow p_{G}^{*}\left(\widehat{\gamma}_{G}^{\ell}\right)\right|_{\Omega^{n-p+1,0}\left(\gamma_{G}^{n}, P\right)}$,

which are defined by, for $x \in N, \mathbf{v} \in T_{x} N, \mathbf{w} \in\left(\nu_{N}^{\ell}\right)_{x}$,

$$
B_{T N}^{\Omega}(x, \mathbf{v})=\left(j_{x}^{2} f, B_{T N}(\mathbf{v})\right) \quad \text { and } \quad B_{\nu_{N}}^{\Omega}(x, \mathbf{w})=\left(j_{x}^{2} f, B_{\nu_{N}}(\mathbf{w})\right)
$$

respectively. We now define $\mu_{n, p}^{G}(f)$ by

$$
\mu_{n, p}^{G}(f)=\left[T\left(B_{\nu_{N}}^{\Omega}\right) \circ \alpha_{N}\right] .
$$

Since all Riemannian metrics on a manifold are homotopic each other and $\ell \gg$ $n, \mu_{n, p}^{G}(f)$ does not depend on choices of Riemannian metrics of $N$ and $P$, and of an embedding $N \rightarrow \mathbf{R}^{n+\ell}$. It is easy to see that $\left(\lim _{\ell \rightarrow \infty} T\left(B_{\widehat{\gamma}^{\ell}}\right)\right)_{*}\left(\mu_{n, p}^{\widetilde{G}_{n, \ell}}(f)\right)=$ $\mu_{n, p}^{G_{n, \ell}}(f)$.

Next let $a: S^{n+\ell} \rightarrow T\left(\left.p_{G}^{*}\left(\widehat{\gamma}_{G}^{\ell}\right)\right|_{\Omega^{n-p+1,0}\left(\gamma_{G}^{n}, P\right)}\right)$ be a map. We may suppose that $a$ is smooth around $a^{-1}\left(\Omega^{n-p+1,0}\left(\gamma_{G}^{n}, T P\right)\right)$ and is transverse to 
$\Omega^{n-p+1,0}\left(\gamma_{G}^{n}, T P\right)$. Let $N$ be the submanifold $a^{-1}\left(\Omega^{n-p+1,0}\left(\gamma_{G}^{n}, T P\right)\right)$ and $\nu_{N}^{\ell}$ be the normal bundle of $N \subset \mathbf{R}^{n+\ell}=S^{n+\ell} \backslash\{$ base point $\}$. Let $B_{\nu_{N}}^{\Omega}(a): \nu_{N}^{\ell} \rightarrow$ $\left.p_{G}^{*}\left(\widehat{\gamma}_{G}^{\ell}\right)\right|_{\Omega^{n-p+1,0}\left(\gamma_{G}^{n}, P\right)}$ be the bundle map induced from the map $a$. By the definition of the structure of $\nu_{N}^{\ell}$ as the normal bundle, we obtain the following homotopy commutative diagram of the exact sequences

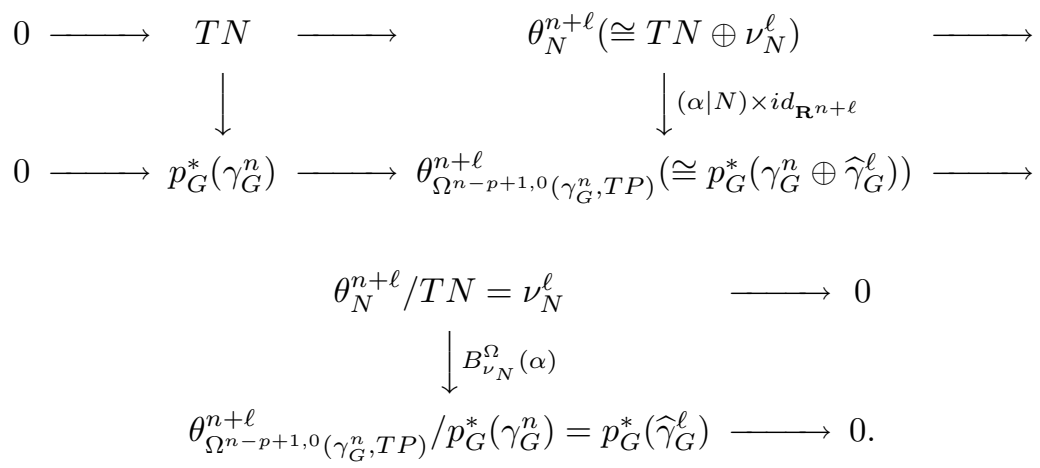

This diagram yields the bundle map $B_{T N}^{\Omega}(a): T N \rightarrow p_{G}^{*}\left(\gamma_{G}^{n}\right)$ covering $a \mid N$ such that $B_{T N}^{\Omega}(a) \oplus B_{\nu_{N}}^{\Omega}(a)$ is homotopic to $(a \mid N) \times i d_{\mathbf{R}^{n+\ell}}$. Therefore, $p_{G} \circ(a \mid N)$ is regarded as the classifying map $c_{N}: N \rightarrow G$. By the commutative diagram (3.7), $a \mid N$ induces a section $s: N \rightarrow \Omega^{n-p+1,0}(T N, T P)\left(\cong \Omega^{n-p+1,0}(N, P)\right)$ such that $B_{\Omega} \circ s=a \mid N$. By the homotopy principle for fold-maps in [An4, Theorem 0.5], we obtain a fold-map $f: N \rightarrow P$ such that $j^{2} f$ and $s$ are homotopic as sections $\Gamma(N, P)$. We should note that $c_{N}, B_{T N}$ and $B_{\nu_{N}}$ defined for $f$ are homotopic to $p_{G} \circ(a \mid N), B_{p_{G}^{*}\left(\gamma_{G}^{n}\right)} \circ B_{T N}^{\Omega}(a)$ and $B_{p_{G}^{*}\left(\hat{\gamma}_{G}^{\ell}\right)} \circ B_{\nu_{N}}^{\Omega}(a)$ respectively. Therefore, we have

$$
\begin{aligned}
\mu_{n, p}^{G}(f) & =\left[T\left(B_{\nu_{N}}^{\Omega}\right) \circ \alpha_{N}\right] \\
& =\left[T\left(B_{\nu_{N}}^{\Omega}(a)\right) \circ \alpha_{N}\right] \\
& =[a] .
\end{aligned}
$$

This concludes the assertion.

\section{$\S 4 . \quad$ Dual Spaces and Duality Isomorphisms}

In this section we study $\lim _{k \rightarrow \infty} \pi_{n+k+\ell}\left(T\left(\nu_{P}^{k}\right) \wedge T\left(\widehat{\gamma}_{\widetilde{G}_{n-p+1, \ell}^{\ell}}\right)\right)$ by using S-dual spaces and duality maps in the suspension category due to [Sp1] and [Sp2]. Let $S^{\ell}$ be the sphere with radius 1 centred at the origin in $\mathbf{R}^{\ell+1}$ with base point $(1,0, \ldots, 0)$. We identify $S^{\ell}$ with the wedge product $S^{1} \wedge \cdots \wedge S^{1}$ of $\ell$ copies of $S^{1}$. We denote the set of homotopy classes of maps $\alpha: A \rightarrow B$ 
by $[A, B]$. Let $A$ be a finite polyhedron with base point. According to [Sp2], $S^{\ell} A$ denotes the $\ell$-th suspension $A \wedge S^{\ell}$. Let $S^{\ell}(c)$ denote the $\ell$-th suspension of a map $c$. If $B$ is also a finite polyhedron with base point, then we denote, by $\{A, B\}$, the set of S-homotopy classes of S-maps, which preserve base points. An element of $\{A, B\}$ represented by a map $\alpha: S^{\ell} A \rightarrow S^{\ell} B(\ell \geq 0)$ is written as $\{\alpha\}$. Let $i_{A, B}: A \wedge B \rightarrow B \wedge A$ be the map defined by $i_{A, B}(x, y)=(y, x)$.

An m-duality map $v: A \wedge B \rightarrow S^{m}$ refers to a continuous map such that the map $\varphi_{v}: H_{q}(A ; \mathbf{Z}) \rightarrow H^{m-q}(B ; \mathbf{Z})$ defined by sending $z \in H_{q}(A ; \mathbf{Z})$ to the slant product $(v)^{*}\left(\left[S^{m}\right]^{*}\right) / z$ is an isomorphism. The duality map of the identification $S^{k} \wedge S^{m} \rightarrow S^{k+m}$ is denoted by $i_{\mathbf{S}}$ for any dimensions $k$ and $m$.

Let $\mathcal{G}=\widetilde{G}_{n-p+1, \ell}$ and $\mathfrak{G}=\widetilde{G}_{n-p, \ell}$ in this section. Given a vector bundle $\xi$ over $X$, we have that $T\left(\xi \oplus \theta_{X}\right)$ is canonically homeomorphic to $T(\xi) \wedge S^{1}$. Hence we write $T\left(\xi \oplus \theta_{X}\right)=T(\xi) \wedge S^{1}$. Under this identification, we have the following bijections for $X=\widetilde{G}_{n-p+1, \ell}$ or $\widetilde{G}_{n-p, \ell}(\ell \gg n)$.

$$
\Pi_{X}: \lim _{k \rightarrow \infty} \pi_{n+k+\ell}\left(T\left(\nu_{P}^{k}\right) \wedge T\left(\widehat{\gamma}_{X}^{\ell}\right)\right) \rightarrow\left\{S^{n+k+\ell} ; T\left(\nu_{P}^{k}\right) \wedge T\left(\widehat{\gamma}_{X}^{\ell}\right)\right\} .
$$

Let $P^{0}$ be the disjoint union of $P$ and the base point $*_{P}$. By [M-S, Lemma 2] and [At, Theorem 3.3] there exist duality maps for sufficiently large numbers $k, q$ and $\ell$

$$
\begin{aligned}
v_{P} & :\left(P^{0}\right) \wedge T\left(\nu_{P}^{k}\right) \rightarrow S^{p+k} \\
v_{\mathcal{G}} & : T\left(\gamma_{\mathcal{G}}^{n-p+1} \oplus \nu_{\mathcal{G}}^{q}\right) \wedge T\left(\widehat{\gamma}_{\mathcal{G}}^{\ell}\right) \rightarrow S^{\ell(n-p+1)+\ell+q+n-p+1} \\
v_{\mathfrak{G}} & : T\left(\gamma_{\mathfrak{G}}^{n-p} \oplus \theta_{\mathfrak{G}} \oplus \nu_{\mathfrak{G}}^{q}\right) \wedge T\left(\widehat{\gamma}_{\mathfrak{G}}^{\ell}\right) \rightarrow S^{\ell(n-p)+\ell+q+n-p+1} .
\end{aligned}
$$

By [Spa2, Theorem 6.8] we obtain the following duality maps

$$
\begin{aligned}
& \nu_{P, \mathcal{G}}=\left(v_{P} \wedge v_{\mathcal{G}}\right) \circ\left(i d_{P^{0}} \wedge i_{T\left(\gamma_{\mathcal{G}}^{n-p+1} \oplus \nu_{\mathcal{G}}^{q}\right), T\left(\nu_{P}^{k}\right)}^{\backsim} \wedge i d_{T\left(\widehat{\gamma}_{\mathcal{G}}^{\ell}\right)}\right) \\
& :\left(P^{0}\right) \wedge T\left(\gamma_{\mathcal{G}}^{n-p+1} \oplus \nu_{\mathcal{G}}^{q}\right) \wedge T\left(\nu_{P}^{k}\right) \wedge T\left(\widehat{\gamma}_{\mathcal{G}}^{\ell}\right) \rightarrow S^{\ell(n-p+1)+\ell+q+n+k+1}, \\
& \nu_{P, \mathfrak{G}}=\left(v_{P} \wedge v_{\mathfrak{G}}\right) \circ\left(i d_{P^{0}} \wedge i_{T\left(\gamma_{\mathfrak{G}}^{n-p} \oplus \theta_{\mathfrak{G}} \oplus \nu_{\mathfrak{G}}^{q}\right), T\left(\nu_{P}^{k}\right)} \wedge i d_{T\left(\widehat{\gamma}_{\mathfrak{G}}^{\ell}\right)}\right) \\
& :\left(P^{0}\right) \wedge T\left(\gamma_{\mathfrak{G}}^{n-p} \oplus \theta_{\mathfrak{G}} \oplus \nu_{\mathfrak{G}}^{q}\right) \wedge T\left(\nu_{P}^{k}\right) \wedge T\left(\widehat{\gamma}_{\mathfrak{G}}^{\ell}\right) \rightarrow S^{\ell(n-p)+\ell+q+n+k+1} .
\end{aligned}
$$

Let $\mathcal{D}_{\mathcal{G}}$ and $\mathcal{D}_{\mathfrak{G}}$ denote the following duality isomorphisms respectively with $m=\ell(n-p+1)+\ell+q+n+k+1$

$$
\begin{aligned}
\mathcal{D}_{m}\left(i_{\mathbf{S}}, \nu_{P, \mathcal{G}}\right) & :\left\{S^{n+k+\ell} ; T\left(\nu_{P}^{k}\right) \wedge T\left(\widehat{\gamma}_{\mathcal{G}}^{\ell}\right)\right\} \\
& \rightarrow\left\{\left(P^{0}\right) \wedge T\left(\gamma_{\mathcal{G}}^{n-p+1} \oplus \nu_{\mathcal{G}}^{q}\right) ; S^{\ell(n-p+1)+q+1}\right\}, \\
\mathcal{D}_{m}\left(i_{\mathbf{S}}, S^{\ell}\left(\nu_{P, \mathfrak{G}}\right)\right) & :\left\{S^{n+k+\ell} ; T\left(\nu_{P}^{k}\right) \wedge T\left(\widehat{\gamma}_{\mathfrak{G}}^{\ell}\right)\right\} \\
& \rightarrow\left\{\left(P^{0}\right) \wedge S^{\ell} T\left(\gamma_{\mathfrak{G}}^{n-p} \oplus \theta_{\mathfrak{G}} \oplus \nu_{\mathfrak{G}}^{q}\right) ; S^{\ell(n-p+1)+q+1}\right\},
\end{aligned}
$$


which are defined as follows. Let $c: S^{n+k+\ell} \rightarrow T\left(\nu_{P}^{k}\right) \wedge T\left(\hat{\gamma}_{\mathcal{G}}^{\ell}\right)$ represent a map in $\left\{S^{n+k+\ell} ; T\left(\nu_{P}^{k}\right) \wedge T\left(\widehat{\gamma}_{\mathcal{G}_{j}}^{\ell}\right)\right\}$. Then $\mathcal{D}_{\mathcal{G}}(\{c\})$ is represented by the map

$$
\begin{aligned}
\nu_{P, \mathcal{G}} \circ\left(i d_{\left(P^{0}\right) \wedge T\left(\gamma_{\mathcal{G}}^{n-p+1} \oplus \nu_{\mathcal{G}}^{q}\right)} \wedge c\right): & \left(P^{0}\right) \wedge T\left(\gamma_{\mathcal{G}}^{n-p+1} \oplus \nu_{\mathcal{G}}^{q}\right) \wedge S^{n+k+\ell} \\
& \rightarrow\left(P_{0}\right) \wedge T\left(\gamma_{\mathcal{G}}^{n-p+1} \oplus \nu_{\mathcal{G}}^{q}\right) \wedge T\left(\nu_{P}^{k}\right) \wedge T\left(\hat{\gamma}_{\mathcal{G}}^{\ell}\right) \\
& \rightarrow S^{\ell(n-p)+\ell+q+n+k+1} .
\end{aligned}
$$

The definition of $\mathcal{D}_{\mathfrak{G}}$ is similar.

Let $C_{0}\left(T\left(\gamma_{\mathcal{G}}^{n-p+1} \oplus \nu_{\mathcal{G}}^{q}\right), S^{\ell(n-p+1)+q+1}\right)$ and $C_{0}\left(T\left(\gamma_{\mathfrak{G}}^{n-p} \oplus \theta_{\mathfrak{G}} \oplus \nu_{\mathfrak{G}}^{q}\right)\right.$, $\left.S^{\ell(n-p)+q+1}\right)$ denote the space of all base point preserving continuous maps $T\left(\gamma_{\mathcal{G}}^{n-p+1} \oplus \nu_{\mathcal{G}}^{q}\right) \rightarrow S^{\ell(n-p+1)+q+1}$ and $T\left(\gamma_{\mathfrak{G}}^{n-p} \oplus \theta_{\mathfrak{G}} \oplus \nu_{\mathfrak{G}}^{q}\right) \rightarrow S^{\ell(n-p)+q+1}$ equipped with the compact-open topology respectively. With the identification $T\left(\xi \oplus \theta_{X}\right)=T(\xi) \wedge S^{1}$ we have the map

$$
\begin{aligned}
& C_{0}\left(T\left(\gamma_{\mathcal{G}}^{n-p+1} \oplus \nu_{\mathcal{G}}^{q}\right),\right.\left.S^{\ell(n-p+1)+q+1}\right) \\
& \rightarrow C_{0}\left(T\left(\gamma_{\mathcal{G}}^{n-p+1} \oplus \nu_{\mathcal{G}}^{q} \oplus \theta_{\mathcal{G}}\right), S^{\ell(n-p+1)+q+2}\right), \\
& C_{0}\left(T\left(\gamma_{\mathfrak{G}}^{n-p} \oplus \theta_{\mathfrak{G}} \oplus \nu_{\mathfrak{G}}^{q}\right),\right.\left.S^{\ell(n-p)+q+1}\right) \\
& \rightarrow C_{0}\left(T\left(\gamma_{\mathfrak{G}-p}^{n-p} \oplus \theta_{\mathfrak{G}} \oplus \nu_{\mathfrak{G}}^{q} \oplus \theta_{\mathfrak{G}}\right), S^{\ell(n-p)+q+2}\right)
\end{aligned}
$$

defined by mapping, for example, $c_{\mathcal{G}}$ to $c_{\mathcal{G}} \wedge i d_{S^{1}}$, where $c_{\mathcal{G}}$ is an element of $C_{0}\left(T\left(\gamma_{\mathcal{G}}^{n-p+1} \oplus \nu_{\mathcal{G}}^{q}\right), S^{\ell(n-p+1)+q+1}\right)$. Let $C_{0}\left(\mathbf{T}_{\mathcal{G}}, \mathbf{S}\right)$ and $C_{0}\left(\mathbf{T}_{\mathfrak{G}}, \mathbf{S}\right)$ be the space defined by

$$
\begin{aligned}
& C_{0}\left(\mathbf{T}_{\mathcal{G}}, \mathbf{S}\right)=\lim _{q \rightarrow \infty} C_{0}\left(T\left(\gamma_{\mathcal{G}}^{n-p+1} \oplus \nu_{\mathcal{G}}^{q}\right), S^{\ell(n-p+1)+q+1}\right), \\
& C_{0}\left(\mathbf{T}_{\mathfrak{G}}, \mathbf{S}\right)=\lim _{q \rightarrow \infty} C_{0}\left(T\left(\gamma_{\mathfrak{G}}^{n-p} \oplus \theta_{\mathfrak{G}} \oplus \nu_{\mathfrak{G}}^{q}\right), S^{\ell(n-p)+q+1}\right)
\end{aligned}
$$

respectively. Then we define the bijections

$$
\begin{aligned}
& \mathbf{i}_{P, \mathcal{G}}:\left\{\left(P^{0}\right) \wedge T\left(\gamma_{\mathcal{G}}^{n-p+1} \oplus \nu_{\mathcal{G}}^{q}\right) ; S^{\ell(n-p+1)+q+1}\right\} \rightarrow\left[P, C_{0}\left(\mathbf{T}_{\mathcal{G}}, \mathbf{S}\right)\right], \\
& \mathbf{i}_{P, \mathfrak{G}}:\left\{\left(P^{0}\right) \wedge S^{\ell} T\left(\gamma_{\mathfrak{G}}^{n-p} \oplus \theta_{\mathfrak{G}} \oplus \nu_{\mathfrak{G}}^{q}\right) ; S^{\ell(n-p+1)+q+1}\right\} \rightarrow\left[P, C_{0}\left(\mathbf{T}_{\mathfrak{G}}, \mathbf{S}\right)\right],
\end{aligned}
$$

by $\mathbf{i}_{P, \mathcal{G}}\left(c_{P, \mathcal{G}}\right)(x)=\left[c_{P, \mathcal{G}} \mid\left(x \cup *_{P}\right) \wedge T\left(\gamma_{\mathcal{G}}^{n-p+1} \oplus \nu_{\mathcal{G}}^{q}\right)\right]$ and $\mathbf{i}_{P, \mathfrak{G}}\left(c_{P, \mathfrak{G}}\right)(x)=$ $\left[c_{P, \mathfrak{G}} \mid\left(x \cup *_{P}\right) \wedge S^{\ell} T\left(\gamma_{\mathfrak{G}}^{n-p} \oplus \theta_{\mathfrak{G}} \oplus \nu_{\mathfrak{G}}^{q}\right)\right]$, where $c_{P, \mathcal{G}}$ and $c_{P, \mathfrak{G}}$ represents elements $\left\{\left(P^{0}\right) \wedge T\left(\gamma_{\mathcal{G}}^{n-p+1} \oplus \nu_{\mathcal{G}}^{q}\right) ; S^{\ell(n-p+1)+q+1}\right\},\left\{\left(P^{0}\right) \wedge S^{\ell} T\left(\gamma_{\mathfrak{G}}^{n-p} \oplus \theta_{\mathfrak{G}} \oplus\right.\right.$ $\left.\left.\nu_{\mathfrak{G}}^{q}\right) ; S^{\ell(n-p+1)+q+1}\right\}$ and $x \in P$ respectively.

Set $\mathcal{D}_{\mathcal{G}, \mathfrak{G}}=\mathcal{D}_{\ell(n-p+1)+\ell+q+n-p+1}\left(v_{\mathcal{G}}, S^{\ell}\left(v_{\mathfrak{G}}\right)\right)$. Let $\mathcal{D}_{\mathcal{G}, \mathfrak{G}}\left(\left\{T\left(\widehat{i_{\mathfrak{G}, \mathcal{G}}}\right)\right\}\right) \in$ $\left\{T\left(\nu_{\mathcal{G}}^{q} \oplus \gamma_{\mathcal{G}}^{n-p+1}\right) ; S^{\ell} T\left(\gamma_{\mathfrak{G}}^{n-p} \oplus \theta_{\mathfrak{G}} \oplus \nu_{\mathfrak{G}}^{q}\right)\right\}$ be the dual map of $T\left(\widehat{i_{\mathfrak{G}, \mathcal{G}}}\right): T\left(\widehat{\gamma}_{\mathfrak{G}}^{\ell}\right)$ $\rightarrow T\left(\widehat{\gamma}_{\mathcal{G}}^{\ell}\right)$. We define the map

$$
\mathcal{D}_{\mathcal{G}, \mathfrak{G}}\left(\left\{T\left(\widehat{i_{\mathfrak{G}, \mathcal{G}}}\right)\right\}\right)_{*}:\left[P, C_{0}\left(\mathbf{T}_{\mathfrak{G}}, \mathbf{S}\right)\right] \rightarrow\left[P, C_{0}\left(\mathbf{T}_{\mathcal{G}}, \mathbf{S}\right)\right]
$$


Let $C_{\mathfrak{G}, \mathcal{G}}: T\left(\gamma_{\mathcal{G}}^{n-p+1} \oplus \nu_{\mathcal{G}}^{q}\right) \rightarrow S^{\ell} T\left(\gamma_{\mathfrak{G}}^{n-p} \oplus \theta_{\mathfrak{G}} \oplus \nu_{\mathfrak{G}}^{q}\right)$ represent $\mathcal{D}_{\mathcal{G}, \mathfrak{G}}\left(\left\{T\left(\widehat{i_{\mathfrak{G}, \mathcal{G}}}\right)\right\}\right)$. For an element $c_{\mathfrak{G}} \in\left[P, C_{0}\left(T\left(\gamma_{\mathfrak{G}}^{n-p} \oplus \theta_{\mathfrak{G}} \oplus \nu_{\mathfrak{G}}^{q}\right), S^{\ell(n-p)+q+1}\right)\right]$ we set $\mathcal{D}_{\mathcal{G}, \mathfrak{G}}\left(\left\{T\left(\widehat{i_{\mathfrak{G}, \mathcal{G}}}\right)\right\}\right)_{*}\left(c_{\mathfrak{G}}\right)(x)=\left[c_{\mathfrak{G}}(x) \circ C_{\mathcal{G}, \mathfrak{G}}\right]$, where $x \in P$. It is obvious that this definition is well defined.

We have the following proposition.

Proposition 4.1. Let $\ell \gg n$ and let $\mathcal{G}=\widetilde{G}_{n-p+1, \ell}$ and $\mathfrak{G}=\bar{G}_{n-p, \ell}$. Then we have the commutative diagram

$$
\begin{array}{ccc}
\lim _{k \rightarrow \infty} \pi_{n+k+\ell}\left(T\left(\nu_{P}^{k}\right) \wedge T\left(\widehat{\gamma}_{\mathfrak{G}}^{\ell}\right)\right) \stackrel{\mathbf{i}_{P, \mathfrak{G}} \circ \mathcal{D}_{\mathfrak{G}} \circ \Pi_{\mathfrak{G}}}{\longrightarrow}\left[P, C_{0}\left(\mathbf{T}_{\mathfrak{G}}, \mathbf{S}\right)\right] \\
\left(i d_{T\left(\nu_{P}^{k}\right)} \wedge T\left(\widehat{i_{\mathfrak{G}, \mathcal{G}}}\right)\right)_{*} \downarrow & \downarrow \mathcal{D}_{\mathcal{G}, \mathfrak{G}}\left(\left\{T\left(\widehat{i_{\mathfrak{G}, \mathcal{G}}}\right)\right\}\right)_{*} \\
\lim _{k \rightarrow \infty} \pi_{n+k+\ell}\left(T\left(\nu_{P}^{k}\right) \wedge T\left(\widehat{\gamma}_{\mathcal{G}}^{\ell}\right)\right) & \underset{\mathbf{i}_{P, \mathcal{G}} \circ \mathcal{D}_{\mathcal{G}} \circ \Pi_{\mathcal{G}}}{\longrightarrow}\left[P, C_{0}\left(\mathbf{T}_{\mathcal{G}}, \mathbf{S}\right)\right],
\end{array}
$$

where $\mathbf{i}_{P, \mathfrak{G}} \circ \mathcal{D}_{\mathfrak{G}} \circ \Pi_{\mathfrak{G}}$ and $\mathbf{i}_{P, \mathcal{G}} \circ \mathcal{D}_{\mathcal{G}} \circ \Pi_{\mathcal{G}}$ are bijective.

Proof. We set $\mathcal{D}_{P}=\mathcal{D}_{p+k}\left(v_{P}, v_{P}\right):\left\{T\left(\nu_{P}^{k}\right) ; T\left(\nu_{P}^{k}\right)\right\} \rightarrow\left\{P^{0} ; P^{0}\right\}$. By (4.1) we have

$$
\begin{aligned}
& \left(\mathcal{D}_{\mathcal{G}, \mathfrak{G}}\left(\left\{T\left(\widehat{i_{\mathfrak{G}, \mathcal{G}}}\right)\right\}\right)_{*} \circ \mathbf{i}_{P, \mathfrak{G}} \circ \mathcal{D}_{\mathfrak{G}} \circ \Pi_{\mathfrak{G}}(c)\right)(x) \\
& =\left[\mathcal{D}_{\mathfrak{G}}(c) \circ\left(i d_{P^{0}} \wedge \mathcal{D}_{\mathcal{G}, \mathfrak{G}}\left(\left\{T\left(\widehat{i_{\mathfrak{G}, \mathcal{G}}}\right)\right\}\right) \mid\left(x \cup *_{P}\right) \wedge T\left(\nu_{\mathcal{G}}^{q} \oplus \gamma_{\mathcal{G}}^{n-p+1}\right)\right],\right.
\end{aligned}
$$

and

$$
\begin{aligned}
& \left(\mathbf{i}_{P, \mathcal{G}} \circ \mathcal{D}_{\mathcal{G}} \circ \Pi_{\mathcal{G}} \circ\left(i d_{T\left(\nu_{P}^{k}\right)} \wedge T\left(\widehat{i_{\mathfrak{G}, \mathcal{G}}}\right)\right)_{*}(c)\right)(x) \\
& =\left[\mathcal{D}_{\mathcal{G}}\left(\left\{\left(i d_{T\left(\nu_{P}^{k}\right)} \wedge T\left(\widehat{i_{\mathfrak{G}, \mathcal{G}}}\right)\right) \circ c\right\}\right) \mid\left(x \cup *_{P}\right) \wedge T\left(\nu_{\mathcal{G}}^{q} \oplus \gamma_{\mathcal{G}}^{n-p+1}\right)\right] .
\end{aligned}
$$

Since we have

$$
\begin{aligned}
& \mathcal{D}_{\mathfrak{G}}(\{c\}) \circ\left(i d_{P^{0}} \wedge \mathcal{D}_{\mathcal{G}, \mathfrak{G}}\left(\left\{T\left(\widehat{i_{\mathfrak{G}, \mathcal{G}}}\right)\right\}\right)\right. \\
& =\mathcal{D}_{\mathfrak{G}}(\{c\}) \circ\left(\mathcal{D}_{P}\left(\left\{i d_{T\left(\nu_{P}^{k}\right)}\right) \wedge \mathcal{D}_{\mathcal{G}, \mathfrak{G}}\left(\left\{T\left(\widehat{i_{\mathfrak{G}, \mathcal{G}}}\right)\right\}\right)\right\}\right) \\
& =\mathcal{D}_{\mathfrak{G}}(\{c\}) \circ \mathcal{D}_{\mathcal{G}}\left(\left\{i d_{T\left(\nu_{P}^{k}\right)} \wedge T\left(\widehat{i_{\mathfrak{G}, \mathcal{G}}}\right)\right\}\right) \\
& =\mathcal{D}_{\mathcal{G}}\left(\left\{\left(i d_{T\left(\nu_{P}^{k}\right)} \wedge T\left(\widehat{i_{\mathfrak{G}, \mathcal{G}}}\right)\right) \circ c\right\}\right)
\end{aligned}
$$

by [Spa2, Theorems 5.11 and 6.3], it follows that maps representing $\mathcal{D}_{\mathfrak{G}}(\{c\}) \circ$ $\left(i d_{P^{0}} \wedge \mathcal{D}_{\mathcal{G}, \mathfrak{G}}\left(\left\{T\left(\widehat{i_{\mathfrak{G}, \mathcal{G}}}\right)\right\}\right)\right.$ and $\mathcal{D}_{\mathcal{G}}\left(\left\{\left(i d_{T\left(\nu_{P}^{k}\right)} \wedge T\left(\widehat{i_{\mathfrak{G}, \mathcal{G}}}\right)\right) \circ c\right\}\right)$ are homotopic. This fact shows the commutativity of the diagram.

Corollary 4.2. Let $\ell \gg n$. Let $f: N \rightarrow P$ be a (resp. quasidefinite) fold-map. Given an element $a \in H^{*}\left(C_{0}\left(\mathbf{T}_{\mathcal{G}}, \mathbf{S}\right)\right)$, the class $\left(\mathbf{i}_{P, \mathcal{G}} \circ \mathcal{D}_{\mathcal{G}} \circ \Pi_{\mathcal{G}} \circ\right.$ $\left.\bar{\omega}_{n, p}^{\Omega}\right)^{*}(a) \in H^{*}(P)$ depends only on the oriented-fold-cobordism class of $f$. 
By Corollary 4.2 it is important to study the structure of the algebra $H^{*}\left(C_{0}\left(\mathbf{T}_{\mathcal{G}}, \mathbf{S}\right)\right)$ for $n>p$.

Remark 4.3. Let $n=p \geq 2$. This case has been dealt with more precisely in [An3], where $\mathcal{G}$ is regarded as a single point. Then we have

$$
C_{0}\left(\mathbf{T}_{\mathcal{G}}, \mathbf{S}\right)=F=\lim _{q \rightarrow \infty} F(q+1),
$$

where $F(q+1)$ is the space of all base point preserving maps of $S^{q}$ equipped with the compact-open topology (see $[\mathrm{At}],[\mathrm{M}-\mathrm{M}]$ and $[\mathrm{Tsu}]$ ). In our case $\mathcal{G}=S^{\ell}$, we have $\gamma_{S^{\ell}}^{1}=\theta_{S^{\ell}}$ and $\nu_{S^{\ell}}^{q}=\theta_{S^{\ell}}^{q}$. Since $T\left(\gamma_{S^{\ell}}^{1} \oplus \nu_{S^{\ell}}^{q}\right)$ is homeomorphic to $\left(S^{\ell}\right)^{0} \wedge S^{q+1}, C_{0}\left(\mathbf{T}_{S^{\ell}}, \mathbf{S}\right)$ is weakly homotopy equivalent to $F$.

The following proposition follows from Propositions 3.3 and 3.4.

Proposition 4.4. Let $\ell \gg n$ and let $\mathcal{G}=\widetilde{G}_{n-p+1, \ell}$ and $\mathfrak{G}=\widetilde{G}_{n-p, \ell}$.

(1) Let $f: N \rightarrow P$ be a submersion. Then $\mathbf{i}_{P, \mathcal{G}} \circ \mathcal{D}_{\mathcal{G}} \circ \Pi_{\mathcal{G}} \circ \bar{\omega}_{n, p}(f)$ lies in the image of $\mathcal{D}_{\mathcal{G}, \mathfrak{G}}\left(\left\{T\left(\widehat{i_{\mathfrak{G}, \mathcal{G}}}\right)\right\}\right)_{*}$.

(2) Let $L$ and $p_{P}: L \times P \rightarrow P$ be as in Proposition 3.4. Then $\mathbf{i}_{P, \mathcal{G}} \circ \mathcal{D}_{\mathcal{G}} \circ$ $\Pi_{\mathcal{G}} \circ \bar{\omega}_{n, p}\left(p_{P}\right)$ is homotopic to the constant map with value $\mathcal{D}_{\mathcal{G}}\left(\left\{T\left(\widehat{i_{\mathfrak{G}, \mathcal{G}}}\right) \circ\right.\right.$ $\left.\left.T\left(\widetilde{c_{\nu_{L}^{k}}}\right) \circ \alpha_{L}\right\}\right)$ in $C_{0}\left(\mathbf{T}_{\mathcal{G}}, \mathbf{S}\right)$.

\section{$\S 5 . \quad$ Lemmas}

Let $A$ be a $p \times n$ matrix, where $n \geq p$. Then $A^{t} A$ is a symmetric and nonnegative definite $p \times p$ matrix. Hence, $A^{t} A$ is triangulated by an orthogonal matrix $T$ as $T\left(A^{t} A\right)^{t} T=\Delta\left(d_{1}^{2}, \ldots, d_{p}^{2}\right)$, where $d_{1}, \ldots, d_{p}$ are non-negative real numbers. Suppose that $T A$ is written as $\left(\begin{array}{l}\mathbf{a}_{1} \\ \vdots \\ \mathbf{a}_{p}\end{array}\right)$ by the row vectors $\mathbf{a}_{i}$ $(1 \leq i \leq p)$. Then we have that $\left(\mathbf{a}_{i}, \mathbf{a}_{j}\right)=0$ for $i \neq j$ and $\left(\mathbf{a}_{i}, \mathbf{a}_{i}\right)=d_{i}^{2}$. If $\mathbf{a}_{i} \neq \mathbf{0}$, then set $\mathbf{f}_{i}=\mathbf{a}_{i} /\left\|\mathbf{a}_{i}\right\|$. By choosing row vectors $\mathbf{f}_{j}$ of degree $n$ for numbers $j$ such that $\mathbf{a}_{j}=\mathbf{0}$ properly, we can find orthonormal vectors $\mathbf{f}_{1}, \ldots, \mathbf{f}_{p}$. Then it follows that

$$
T A=\Delta\left(\left\|\mathbf{a}_{1}\right\|, \ldots,\left\|\mathbf{a}_{p}\right\|\right)\left(\begin{array}{l}
\mathbf{f}_{1} \\
\vdots \\
\mathbf{f}_{p}
\end{array}\right) .
$$


Hence, we have

$$
A={ }^{t} T \Delta\left(\left\|\mathbf{a}_{1}\right\|, \ldots,\left\|\mathbf{a}_{p}\right\|\right)\left(\begin{array}{l}
\mathbf{f}_{1} \\
\vdots \\
\mathbf{f}_{p}
\end{array}\right) .
$$

Lemma 5.1. Let $n \geq p \geq 2$. Let $A$ be a $p \times p$ matrix of rank $m$ $(0 \leq m \leq p)$. Then there exist matrices $S \in O(p), M \in O(n)$ and real numbers $d_{1}, \ldots, d_{p}$ such that

(1) $d_{1} \geq \cdots \geq d_{m}>0$ and $d_{m+1}=\cdots=d_{p}=0$,

(2) $A=S \Delta(\mathbf{d}) M\left(\begin{array}{l}1 \\ p\end{array}\right)=S(1, m) \Delta\left(d_{1}, \ldots, d_{m}\right) M\left(\begin{array}{l}1 \\ m\end{array}\right)$,

(3) $d_{1}^{2}, \ldots, d_{p}^{2}$ are eigen-values of $A^{t} A$.

Proof. By (5.1) we can find matrices $S \in O(p)$ and $M \in O(n)$ such that $A$ is expressed by $S \Delta(\mathbf{d}) M\left(\begin{array}{l}1 \\ p\end{array}\right)$. Suppose that $d_{i_{1}} \geq \cdots \geq d_{i_{p}} \geq 0$. Let $P\left(i_{1}, \ldots, i_{p}\right)$ be the permutation matrix in $O(p)$ such that $P\left(i_{1}, \ldots, i_{p}\right)\left(\mathbf{e}_{j}\right)=$ $\mathbf{e}_{i_{j}}$. Then we have that

$$
\begin{aligned}
A & =S \Delta(\mathbf{d}) M\left(\begin{array}{l}
1 \\
p
\end{array}\right) \\
& =S P\left(i_{1}, \ldots, i_{p}\right) \Delta\left(d_{i_{1}}, \ldots, d_{i_{p}}\right)^{t} P\left(i_{1}, \ldots, i_{p}\right) M\left(\begin{array}{l}
1 \\
p
\end{array}\right)
\end{aligned}
$$

since $P\left(i_{1}, \ldots, i_{p}\right) \Delta\left(d_{i_{1}}, \ldots, d_{i_{p}}\right)^{t} P\left(i_{1}, \ldots, i_{p}\right)=\Delta\left(d_{1}, \ldots, d_{p}\right)$.

We say that the diagonal components $\mathbf{d}=\left(d_{1}, \ldots, d_{p}\right)$ are non-negative if $d_{i} \geq 0$ for all $i$ and are decreasing if $d_{1} \geq \cdots \geq d_{p}$. The expression $A=$ $S \Delta(\mathbf{d}) M\left(\begin{array}{l}1 \\ p\end{array}\right)$ will be called a diagonalization of $A$.

Lemma 5.2. Let $\mathbf{d}$ and $\mathbf{d}^{\prime}$ be decreasing diagonal components of degree $\ell$. Suppose that ${ }^{t} T \Delta(\mathbf{d}) T=\Delta\left(\mathbf{d}^{\prime}\right)$ for $T \in O(\ell)$. Then we have the following.

(1) We have $\mathbf{d}=\mathbf{d}^{\prime}$.

(2) Suppose that $\Delta(\mathbf{d})\left(=\Delta\left(\mathbf{d}^{\prime}\right)\right)$ is written as $a_{1} E_{i_{1}} \dot{+} a_{2} E_{i_{2}} \dot{+} \cdots \dot{+} a_{s} E_{i_{s}}$, where $a_{1}, \ldots, a_{s}$ are all distinct and $\ell=i_{1}+\cdots+i_{s}$. Then $T$ is also a matrix of the form $T_{1} \dot{+} \cdots \dot{+} T_{s}$, where $T_{j}$ is of rank $i_{j}$ for every $j$.

Proof. The assertion (1) follows from the fact that the set of eigen values of ${ }^{t} T \Delta(\mathbf{d}) T$ is $\left\{d_{1}, \ldots, d_{p}\right\}$. We write $T=\left(t_{i q}\right)=\left(\overline{\mathbf{t}}_{1}, \ldots, \overline{\mathbf{t}}_{\ell}\right)$. By the assumption ${ }^{t} T \Delta(\mathbf{d}) T=\Delta(\mathbf{d})$, we have

$$
\left({ }^{t}\left(d_{1} t_{1 q}, \ldots, d_{\ell} t_{\ell q}\right), \overline{\mathbf{t}}_{m}\right)=d_{q} \delta_{q m}=d_{q}\left(\overline{\mathbf{t}}_{q}, \overline{\mathbf{t}}_{m}\right) .
$$


In other words,

$$
\left({ }^{t}\left(d_{1} t_{1 q}, \ldots, d_{\ell} t_{\ell q}\right)-d_{q} \overline{\mathbf{t}}_{q}, \overline{\mathbf{t}}_{m}\right)=0 \quad(m=1, \ldots, \ell) .
$$

Since $\overline{\mathbf{t}}_{1}, \ldots, \overline{\mathbf{t}}_{\ell}$ are orthonormal basis of $\mathbf{R}^{\ell}$, it follows that ${ }^{t}\left(d_{1} t_{1 q}, \ldots, d_{\ell} t_{\ell q}\right)-$ $d_{q} \overline{\mathbf{t}}_{q}=\mathbf{0}$ for each $q$. Therefore, if $i_{1}+\cdots+i_{j-1}<q \leq i_{1}+\cdots+i_{j}$ and $r$ does not satisfy $i_{1}+\cdots+i_{j-1}<r \leq i_{1}+\cdots+i_{j}$, then we have $t_{r q}=0$. This implies the assertion (2).

Lemma 5.3. Let $\mathbf{d}$ be decreasing diagonal components of degree $\ell$ given in Lemma $5.2(2)$. For a sequence $\left\{T^{k}\right\}$ in $O(\ell)$ and a sequence of decreasing diagonal components $\left\{\mathbf{d}^{k}\right\}$, assume that the sequence $\left\{{ }^{t} T^{k} \Delta\left(\mathbf{d}^{k}\right) T^{k}\right\}$ converges to $\Delta(\mathbf{d})$. Then we have the following.

(1) $\left\{\mathbf{d}^{k}\right\}$ converges to $\mathbf{d}$.

(2) If a pair $(r, q)$ of numbers does not satisfy the inequality

$$
i_{1}+\cdots+i_{j-1}<r, q \leq i_{1}+\cdots+i_{j}
$$

for every integer $j$ with $1 \leq j \leq s\left(i_{0}=0\right)$, then every sequence $\left\{t_{r q}^{k}\right\}$ made of $(r, q)$ components of $T^{k}$ converges to 0 .

(3) Let $\delta\left(T^{k}\right)=\delta\left(T^{k}\right)_{1} \dot{+} \cdots \dot{+} \delta\left(T^{k}\right)_{s}$ be a matrix made of $T^{k}$ by replacing all $(r, q)$ components described in $(2)$ with 0 , where $\delta\left(T^{k}\right)_{j}$ is of rank $i_{j}$. Then for all numbers $j$ with $a_{j} \neq 0,\left\{{ }^{t} \delta\left(T^{k}\right)_{j} \delta\left(T^{k}\right)_{j}\right\}$ converges to $E_{i_{j}}$.

Proof. The assertion (1) follows from the fact that the set of eigen values of a matrix is continuous with respect to components of matrices ([W, Appendix $\mathrm{V}$, Section 4]). For any positive real number $\varepsilon$, there is a number $k_{0}$ such that if $k>k_{0}$, then we have

$$
\left\|^{t} T^{k} \Delta\left(\mathbf{d}^{k}\right) T^{k}-\Delta(\mathbf{d})\right\|<\varepsilon .
$$

We write $T^{k}=\left(t_{i q}^{k}\right)=\left(\overline{\mathbf{t}}_{1}^{k}, \ldots, \overline{\mathbf{t}}_{\ell}^{k}\right)$. Let $\Upsilon_{q m}$ be the $(q, m)$ component of ${ }^{t} T^{k} \Delta\left(\mathbf{d}^{k}\right) T^{k}-\Delta(\mathbf{d})$. Then we have

$$
\Upsilon_{q m}=\left({ }^{t}\left(d_{1}^{k} t_{1 q}^{k}, \ldots, d_{\ell}^{k} t_{\ell q}^{k}\right), \overline{\mathbf{t}}_{m}^{k}\right)-d_{q} \delta_{q m}=\left({ }^{t}\left(d_{1}^{k} t_{1 q}^{k}, \ldots, d_{\ell}^{k} t_{\ell q}^{k}\right)-d_{q} \overline{\mathbf{t}}_{q}^{k}, \overline{\mathbf{t}}_{m}^{k}\right) .
$$

By (5.2), we have $\sum_{m=1}^{\ell} \Upsilon_{q m}^{2}<\varepsilon^{2}$. Since $\overline{\mathbf{t}}_{1}^{k}, \ldots, \overline{\mathbf{t}}_{\ell}^{k}$ is an orthonormal basis, we have that

$$
\sum_{q=1}^{\ell}\left\|^{t}\left(d_{1}^{k} t_{1 q}^{k}, \ldots, d_{\ell}^{k} t_{\ell q}^{k}\right)-d_{q} \overline{\mathbf{t}}_{q}^{k}\right\|^{2}<\varepsilon^{2}
$$


namely

$$
\sum_{m=1}^{\ell}\left(d_{m}^{k}-d_{q}\right)^{2}\left(t_{m q}^{k}\right)^{2}<\varepsilon^{2} .
$$

Setting $V=\min \left\{\left|a_{m}-a_{q}\right| \mid m \neq q\right\}$, and replacing $k_{0}$ by a larger one, we may suppose that $d_{m}^{k}-d_{q} \geq V / 2$. Then we deduce

$$
\left(t_{1 q}^{k}\right)^{2}+\cdots+\left(t_{\left(i_{1}+\cdots+i_{j-1}\right) q}^{k}\right)^{2}+\left(t_{\left(i_{1}+\cdots+i_{j}+1\right) q}^{k}\right)^{2}+\cdots+\left(t_{\ell q}^{k}\right)^{2}<\frac{4 \varepsilon^{2}}{V^{2}} .
$$

If $r$ and $q$ are such numbers given in (2), then the sequence $\left\{t_{r q}^{k}\right\}$ converges to 0 . This is what we want to prove.

Lemma 5.4. Let $n, p$ be integers with $n \geq p \geq 2$. Let $S, S^{\prime} \in O(p)$ and $M, M^{\prime} \in O(n)$ and let $\mathbf{d}=\left(d_{1}, \ldots, d_{p}\right)$ be non-negative and decreasing diagonal components with $d_{p-1}>0$ such that $\Delta(\mathbf{d})$ is written as $a_{1} E_{i_{1}} \dot{+} a_{2} E_{i_{2}} \dot{+} \cdots \dot{+}$ $a_{s} E_{i_{s}}$, where $a_{1}, \ldots, a_{s}$ are all distinct and $p=i_{1}+\cdots+i_{s}$. Assume that $S \Delta(\mathbf{d}) M\left(\begin{array}{l}1 \\ p\end{array}\right)=S^{\prime} \Delta(\mathbf{d}) M^{\prime}\left(\begin{array}{l}1 \\ p\end{array}\right)$. Then we have the following.

(1) If $d_{p}>0$, then there exist matrices $G_{j} \in O\left(i_{j}\right)(1 \leq j \leq s)$ such that $S^{\prime}=S\left({ }^{t} G_{1} \dot{+} \cdots \dot{+}{ }^{t} G_{s-1} \dot{+}{ }^{t} G_{s}\right)$ and $M^{\prime}\left(\begin{array}{l}1 \\ p\end{array}\right)=\left(G_{1} \dot{+} \cdots \dot{+} G_{s-1} \dot{+} G_{s}\right) M\left(\begin{array}{l}1 \\ p\end{array}\right)$.

(2) If $d_{p}=0$ and $i_{s}=1$, then there exist matrices $G_{j} \in O\left(i_{j}\right)(1 \leq j \leq s)$ such that $S^{\prime}=S\left({ }^{t} G_{1} \dot{+} \cdots \dot{+}{ }^{t} G_{s-1}+{ }^{t} G_{s}\right)$ and $M^{\prime}\left(\begin{array}{c}1 \\ p-1\end{array}\right)=\left(G_{1} \dot{+} \cdots \dot{+}\right.$ $\left.G_{s-1}\right) M\left({ }_{p-1}^{1}\right)$.

Proof. We prove the case $d_{p}=0$ and leave the proof for the case $d_{p}>0$ to the reader, since it is similar and easier. So let $d_{p-1}>0$ and $d_{p}=0$.

By the assumption of $S \Delta(\mathbf{d}) M\left(\begin{array}{l}1 \\ p\end{array}\right)=S^{\prime} \Delta(\mathbf{d}) M^{\prime}\left(\begin{array}{l}1 \\ p\end{array}\right)$, we have

$$
{ }^{t} S S^{\prime} \Delta\left(d_{1}, \ldots, d_{p}\right) M^{\prime}\left(\begin{array}{l}
1 \\
p
\end{array}\right)^{t} M=\left(\Delta\left(d_{1}, \ldots, d_{p}\right), \mathbf{0}_{p \times(n-p)}\right) .
$$

Writing both terms $A$ and calculating $A^{t} A$ we deduce

$$
{ }^{t} S S^{\prime} \Delta\left(d_{1}^{2}, \ldots, d_{p}^{2}\right)^{t} S^{\prime} S=\Delta\left(d_{1}^{2}, \ldots, d_{p}^{2}\right) .
$$

Since $\Delta(\mathbf{d})$ is written as $a_{1} E_{i_{1}} \dot{+} a_{2} E_{i_{2}} \dot{+} \cdots \dot{+} a_{s} E_{i_{s}}$, it follows that there exists a decomposition of ${ }^{t} S^{\prime} S$ into $G_{1} \dot{+} \cdots \dot{+} G_{s-1} \dot{+} G_{s}$ with the properties described in Lemma $5.2(2)$, where $G_{j}$ is of rank $i_{j}(1 \leq j \leq s)$. Hence, we have $S^{\prime}=S\left({ }^{t} G_{1} \dot{+} \cdots \dot{+}{ }^{t} G_{s-1} \dot{+}{ }^{t} G_{s}\right)$. 
Furthermore, we obtain that

$$
\begin{aligned}
{ }^{t} S S^{\prime} & \Delta\left(d_{1}, \ldots, d_{p}\right) M^{\prime}\left(\begin{array}{l}
1 \\
p
\end{array}\right)^{t} M \\
& =\left({ }^{t} G_{1} \dot{+} \cdots \dot{+}{ }^{t} G_{s-1} \dot{+}^{t} G_{s}\right) \Delta\left(d_{1}, \ldots, d_{p}\right) M^{\prime}\left(\begin{array}{l}
1 \\
p
\end{array}\right)^{t} M \\
& =\Delta\left(d_{1}, \ldots, d_{p}\right)\left({ }^{t} G_{1}+\cdots+\dot{+}^{t} G_{s-1}+{ }^{t} G_{s}\right) M^{\prime}\left(\begin{array}{l}
1 \\
p
\end{array}\right)^{t} M \\
& =\left(\Delta\left(d_{1}, \ldots, d_{p}\right), \mathbf{0}_{p \times(n-p)}\right) .
\end{aligned}
$$

This induces

$$
\left({ }^{t} G_{1}+\cdots+{ }^{t} G_{s-1}\right) M^{\prime}\left({ }_{p-1}^{1}\right)^{t} M=\left(E_{p-1}, \mathbf{0}_{(p-1) \times(n-p+1)}\right) .
$$

Hence, we have $\left({ }^{t} G_{1} \dot{+} \cdots+{ }^{t} G_{s-1}\right) M^{\prime}\left({ }_{p-1}^{1}\right)=\left(E_{p-1}, \mathbf{0}_{(p-1) \times(n-p+1)}\right) M=$ $M\left({ }_{p-1}^{1}\right)$.

Lemma 5.5. Let $n \geq p \geq 2$ and let $c, d$ be non-negative integers with $n-p+1=c+d$. Let $(\mathbf{v}, \mathbf{w})=\left(v_{1}, \ldots, v_{c}, w_{1}, \ldots, w_{d}\right)$ be diagonal components with $v_{1} \geq \cdots \geq v_{c}>0>w_{1} \geq \cdots \geq w_{d}$ and let $M, M^{\prime}$ be elements of $O(n)$.

(1) If ${ }^{t} M\left(\begin{array}{l}p \\ n\end{array}\right) \Delta(\mathbf{v}, \mathbf{w}) M\left(\begin{array}{l}p \\ n\end{array}\right)={ }^{t} M^{\prime}\left(\begin{array}{l}p \\ n\end{array}\right) \Delta(\mathbf{v}, \mathbf{w}) M^{\prime}\left(\begin{array}{l}p \\ n\end{array}\right)$, then there exist matrices $T_{1} \in O(c), T_{2} \in O(d)$ such that

$$
M^{\prime}\left(\begin{array}{l}
p \\
n
\end{array}\right)=\left(T_{1}+T_{2}\right) M\left(\begin{array}{l}
p \\
n
\end{array}\right) .
$$

(2) If $c=d$ and ${ }^{t} M\left(\begin{array}{l}p \\ n\end{array}\right) \Delta(\mathbf{v}, \mathbf{w}) M\left(\begin{array}{l}p \\ n\end{array}\right)={ }^{t} M^{\prime}\left(\begin{array}{l}p \\ n\end{array}\right) \Delta(\mathbf{w}, \mathbf{v}) M^{\prime}\left(\begin{array}{l}p \\ n\end{array}\right)$, then there exist matrices $T_{1}, T_{2} \in O(c)$ such that

$$
M^{\prime}\left(\begin{array}{l}
p \\
n
\end{array}\right)=\left(\begin{array}{cc}
\mathbf{0} & E_{c} \\
E_{c} & \mathbf{0}
\end{array}\right)\left(T_{1}+T_{2}\right) M\left(\begin{array}{l}
p \\
n
\end{array}\right) .
$$

Proof.

(1) Since $M\left(\begin{array}{l}p \\ n\end{array}\right)^{t} M\left(\begin{array}{l}p \\ n\end{array}\right)=M^{\prime}\left(\begin{array}{l}p \\ n\end{array}\right)^{t} M^{\prime}\left(\begin{array}{l}p \\ n\end{array}\right)=E_{n-p+1}$, we have

$$
M^{\prime}\left(\begin{array}{l}
p \\
n
\end{array}\right)^{t} M\left(\begin{array}{l}
p \\
n
\end{array}\right) \Delta(\mathbf{v}, \mathbf{w}) M\left(\begin{array}{l}
p \\
n
\end{array}\right)^{t} M^{\prime}\left(\begin{array}{l}
p \\
n
\end{array}\right)=\Delta(\mathbf{v}, \mathbf{w}) .
$$

Since

$$
\begin{aligned}
M^{\prime}\left(\begin{array}{c}
1 \\
p-1
\end{array}\right){ }^{t} M^{\prime}\left(\begin{array}{l}
p \\
n
\end{array}\right) \Delta(\mathbf{v}, \mathbf{w}) M^{\prime}\left(\begin{array}{l}
p \\
n
\end{array}\right) & =\mathbf{0}_{(p-1) \times(n-p+1)} \\
& =M^{\prime}\left(\begin{array}{c}
1 \\
p-1
\end{array}\right)^{t} M\left(\begin{array}{l}
p \\
n
\end{array}\right) \Delta(\mathbf{v}, \mathbf{w}) M\left(\begin{array}{l}
p \\
n
\end{array}\right),
\end{aligned}
$$

we have $M^{\prime}\left(\begin{array}{c}1 \\ p-1\end{array}\right)^{t} M\left(\begin{array}{l}p \\ n\end{array}\right)=\mathbf{0}_{(p-1) \times(n-p+1)}$. Furthermore, we have ${ }^{t} M^{\prime} M^{\prime}=$ $E_{n}={ }^{t} M^{\prime}\left(\begin{array}{c}1 \\ p-1\end{array}\right) M^{\prime}\left(\begin{array}{c}1 \\ p-1\end{array}\right)+{ }^{t} M^{\prime}\left(\begin{array}{l}p \\ n\end{array}\right) M^{\prime}\left(\begin{array}{l}p \\ n\end{array}\right)$. We show $M\left(\begin{array}{l}p \\ n\end{array}\right)^{t} M^{\prime}\left(\begin{array}{l}p \\ n\end{array}\right) \in O(n-$ 
$p+1)$. Indeed, we have

$$
\begin{aligned}
{ }^{t}\left(M^{\prime}\left(\begin{array}{l}
p \\
n
\end{array}\right)^{t} M\left(\begin{array}{l}
p \\
n
\end{array}\right)\right) & M^{\prime}\left(\begin{array}{l}
p \\
n
\end{array}\right)^{t} M\left(\begin{array}{l}
p \\
n
\end{array}\right) \\
& =M\left(\begin{array}{l}
p \\
n
\end{array}\right)^{t} M^{\prime}\left(\begin{array}{l}
p \\
n
\end{array}\right) M^{\prime}\left(\begin{array}{c}
p \\
n
\end{array}\right){ }^{t} M\left(\begin{array}{l}
p \\
n
\end{array}\right) \\
& =M\left(\begin{array}{l}
p \\
n
\end{array}\right)\left(E_{n}-{ }^{t} M^{\prime}\left(\begin{array}{c}
1 \\
p-1
\end{array}\right) M^{\prime}\left(\begin{array}{c}
1 \\
p-1
\end{array}\right)\right)^{t} M\left(\begin{array}{l}
p \\
n
\end{array}\right) \\
& =M\left(\begin{array}{l}
p \\
n
\end{array}\right) E_{n}^{t} M\left(\begin{array}{l}
p \\
n
\end{array}\right)-M\left(\begin{array}{l}
p \\
n
\end{array}\right){ }^{t} M^{\prime}\left(\begin{array}{c}
1 \\
p-1
\end{array}\right) M^{\prime}\left(\begin{array}{c}
1 \\
p-1
\end{array}\right)^{t} M\left(\begin{array}{l}
p \\
n
\end{array}\right) \\
& =E_{n-p+1} .
\end{aligned}
$$

Hence, it follows from Lemma 5.2 that there exist matrices $T_{1} \in O(c)$, $T_{2} \in O(d)$ such that $M^{\prime}\left(\begin{array}{l}p \\ n\end{array}\right)^{t} M\left(\begin{array}{l}p \\ n\end{array}\right)=T_{1} \dot{+} T_{2}$. Thus we have $M^{\prime}\left(\begin{array}{l}p \\ n\end{array}\right)=$ $\left(T_{1}+T_{2}\right) M\left(\begin{array}{l}p \\ n\end{array}\right)$.

(2) The assertion follows from (1) and the fact that

$$
\left(\begin{array}{cc}
\mathbf{0} & E_{c} \\
E_{c} & \mathbf{0}
\end{array}\right) \Delta(\mathbf{v}, \mathbf{w})\left(\begin{array}{cc}
\mathbf{0} & E_{c} \\
E_{c} & \mathbf{0}
\end{array}\right)=\Delta(\mathbf{w}, \mathbf{v}) .
$$

Lemma 5.6. Let $\mathbf{d}$ be non-negative and decreasing diagonal components given in Proposition 5.4. For two sequences $\left\{S^{k}\right\}$ in $O(p),\left\{T^{k}\right\}$ in $O(n)$ and a sequence of non-negative and decreasing diagonal components $\left\{\mathbf{d}^{k}\right\}$ of degree $p$, assume that the sequence $\left\{S^{k} \Delta\left(\mathbf{d}^{k}\right) M^{k}\left(\begin{array}{l}1 \\ p\end{array}\right)\right\}$ converges to $(\Delta(\mathbf{d})$, $\left.\mathbf{0}_{p \times(n-p)}\right)$. Then we have the following.

(1) $\left\{\mathbf{d}^{k}\right\}$ converges to $\mathbf{d}$.

(2) If a pair $(r, q)$ of numbers does not satisfy the inequality

$$
i_{1}+\cdots+i_{j-1}<r, q \leq i_{1}+\cdots+i_{j}
$$

for every integer $j$ with $1 \leq j \leq s$, then every sequence $\left\{s_{r q}^{k}\right\}$ made of $(r, q)$ components of $S^{k}$ converges to 0 .

(3) Let $\delta\left(S^{k}\right)=\delta\left(S^{k}\right)_{1} \dot{+} \cdots \dot{+} \delta\left(S^{k}\right)_{s}$ be a matrix made of $S^{k}$ by replacing every $(r, q)$ components described in (2), in turn with 0 , where $\delta\left(S^{k}\right)_{j}$ is of rank $i_{j}$. Then

(3-i) if $a_{j} \neq 0$ for every number $j$, then $\left\{\delta\left(S^{k}\right) M^{k}\left(\begin{array}{l}1 \\ p\end{array}\right)\right\}$ converges to $\left(E_{p}, \mathbf{0}_{p \times(n-p)}\right)$,

(3-ii) if $a_{s}=0$, then $\left\{\delta\left(S^{k}\right)_{1} \dot{+} \cdots \dot{+} \delta\left(S^{k}\right)_{s-1} M^{k}\left(\begin{array}{c}1 \\ p-1\end{array}\right)\right\}$ converges to $\left(E_{p-1}, \mathbf{0}_{(p-1) \times(n-p+1)}\right)$. 
Proof. Setting $A^{k}=S^{k} \Delta\left(\mathbf{d}^{k}\right) M^{k}\left(\begin{array}{l}1 \\ p\end{array}\right)$, we have $A^{k}\left({ }^{t} A^{k}\right)=S^{k} \Delta\left(\left(d_{1}^{k}\right)^{2}, \ldots\right.$, $\left.\left(d_{p}^{k}\right)^{2}\right)^{t} S^{k}$ and

$$
\lim _{k \rightarrow \infty} S^{k} \Delta\left(\left(d_{1}^{k}\right)^{2}, \ldots,\left(d_{p}^{k}\right)^{2}\right)^{t} S^{k}=\lim _{k \rightarrow \infty} A^{k}\left({ }^{t} A^{k}\right)=A\left({ }^{t} A\right)=\Delta\left(d_{1}^{2}, \ldots, d_{p}^{2}\right) .
$$

The assertion (1) follows from Lemma 5.3 (1). By Lemma 5.3 (2) and (3) there exist matrices $\delta\left(S^{k}\right)=\delta\left(S^{k}\right)_{1} \dot{+} \cdots \dot{+} \delta\left(S^{k}\right)_{s}$ with the property

$$
\lim _{k \rightarrow \infty} S^{k}-\delta\left(S^{k}\right)=\mathbf{0}_{p \times p} .
$$

Then we have

$$
\begin{aligned}
\lim _{k \rightarrow \infty} S^{k} \Delta\left(\mathbf{d}^{k}\right) M^{k}\left(\begin{array}{l}
1 \\
p
\end{array}\right) & =\lim _{k \rightarrow \infty} S^{k}\left(\Delta(\mathbf{d})-\Delta\left(\mathbf{d}-\mathbf{d}^{k}\right)\right) M^{k}\left(\begin{array}{l}
1 \\
p
\end{array}\right) \\
& =\lim _{k \rightarrow \infty} S^{k} \Delta(\mathbf{d}) M^{k}\left(\begin{array}{l}
1 \\
p
\end{array}\right) \\
& =\lim _{k \rightarrow \infty} \delta\left(S^{k}\right) \Delta(\mathbf{d}) M^{k}\left(\begin{array}{l}
1 \\
p
\end{array}\right) \\
& =\lim _{k \rightarrow \infty} \Delta(\mathbf{d}) \delta\left(S^{k}\right) M^{k}\left(\begin{array}{l}
1 \\
p
\end{array}\right) \\
& =\Delta(\mathbf{d})\left(\lim _{k \rightarrow \infty} \delta\left(S^{k}\right) M^{k}\left(\begin{array}{l}
1 \\
p
\end{array}\right)\right) \\
& =\left(\Delta(\mathbf{d}), \mathbf{0}_{p \times(n-p)}\right) .
\end{aligned}
$$

Hence, we have (3-i) and (3-ii).

\section{§6. Homotopy Type of $\Omega^{n-p+1}(n, p)$}

For an integer $p \geq 2$, let $\Delta^{p}(\Omega)$ be the subspace in $\mathbf{R}^{p}$ consisting of all points $\left(d_{1}, \ldots, d_{p}\right)$ such that $d_{1} \geq \cdots \geq d_{p-1}>0$ and $d_{p} \geq 0$ and let $\Delta^{p}(1)$ be the subspace consisting of all points $\left(1, d_{2}, \ldots, d_{p}\right) \in \Delta^{p}(\Omega)$. Let $I_{\Delta}^{p}$ be the subspace in $\Delta^{p}(1)$ consisting of all points $(1, \ldots, 1, b)$ with $0 \leq b \leq 1$ and let $\Delta_{\Sigma}^{p}$ be the subspace consisting of all points $\left(1, d_{2}, \ldots, d_{p-2}, 0,0\right)$ with $1 \geq d_{2} \geq$ $\cdots \geq d_{p-2} \geq 0$. It is clear that $\Delta^{p}(1)$ is a deformation retract of $\Delta^{p}(\Omega)$ by a deformation retraction $\left(d_{1}, \ldots, d_{p}\right) \longmapsto\left((1-\lambda)+\lambda d_{1}\right)^{-1}\left(d_{1}, \ldots, d_{p}\right)$ with $0 \leq \lambda \leq 1$. We show that $\Delta^{p}(1)$ is homeomorphic to $\left(I_{\Delta}^{p} * \Delta_{\Sigma}^{p}\right) \backslash \Delta_{\Sigma}^{p}$, where $*$ refers to the join. Indeed, suppose that an element $\left(1, d_{2}, \ldots, d_{p}\right) \in \Delta^{p}(1)$ is expressed by

$$
\left(1, d_{2}, \ldots, d_{p}\right)=s(1, \ldots, 1, b)+(1-s)\left(1, f_{2}, \ldots, f_{p-2}, 0,0\right) .
$$

Then we have $d_{p-1}=s, d_{p}=s b$ and $d_{i}=s+(1-s) f_{i} \quad(2 \leq i \leq p-2)$. Hence, if $s<1$, then we have $s=d_{p-1}, b=d_{p} / d_{p-1}$ and $f_{i}=\left(d_{i}-d_{p-1}\right) /\left(1-d_{p-1}\right)$ $(2 \leq i \leq p-2)$ and vice versa. 
Let $\alpha$ be an element of $\Omega^{n-p+1}(n, p)$ with diagonalization $S \Delta(\mathbf{d}) M\left(\begin{array}{l}1 \\ p\end{array}\right)$, where $S \in O(p), M \in O(n)$ and $\mathbf{d}=\left(d_{1}, \ldots, d_{p}\right)$ is a decreasing diagonal components with $d_{p-1}>0$ and $d_{p} \geq 0$. Let $\Omega_{\Delta}$ (resp. $\left.\Sigma_{\Delta}\right)$ denote the subset consisting of all elements $\alpha$ with diagonalization $S \Delta(\mathbf{d}) M\left(\begin{array}{l}1 \\ p\end{array}\right)$ such that $\mathbf{d} \in \Delta^{p}(1)$ (resp. $\mathbf{d} \in \Delta^{p}(1)$ with $\left.d_{p}=0\right)$. We define a homotopy $R_{\lambda}^{\prime}: \Omega^{n-p+1}(n, p) \rightarrow$ $\Omega^{n-p+1}(n, p)$ by

$$
R_{\lambda}^{\prime}\left(S \Delta(\mathbf{d}) M\left(\begin{array}{l}
1 \\
p
\end{array}\right)\right)=\left((1-\lambda)+\lambda d_{1}\right)^{-1} S \Delta(\mathbf{d}) M\left(\begin{array}{l}
1 \\
p
\end{array}\right) .
$$

The following lemma is obvious.

Lemma 6.1. The homotopy $R_{\lambda}^{\prime}$ is a deformation retraction of $\Omega^{n-p+1}(n, p)$ to $\Omega_{\Delta}$ such that

(1) $R_{\lambda}^{\prime}$ preserves $\Sigma^{n-p}(n, p)$ and $\Sigma^{n-p+1}(n, p)$ respectively,

(2) $R_{\lambda}^{\prime} \mid \Sigma^{n-p+1}(n, p)$ induces a deformation retraction of $\Sigma^{n-p+1}(n, p)$ to $\Sigma_{\Delta}$.

Let $K^{\prime}(n, p, b)$ for $0<b<1, \Sigma K^{\prime}(n, p)$ and $R^{\prime}(n, p)$ denote the subsets consisting of all elements $\alpha$ with diagonalization $S \Delta\left(\mathbf{d}_{b}\right) M\left(\begin{array}{l}1 \\ p\end{array}\right)$ such that $\mathbf{d}_{b} \in I_{\Delta}^{p}$ with $0<b<1, \mathbf{d}_{0} \in I_{\Delta}^{p}$ and $\mathbf{d}_{1}$ respectively. Let $K^{\prime}(n, p)$ denote the union

$$
\Sigma K^{\prime}(n, p) \bigcup\left(\cup_{b \in(0,1)} K^{\prime}(n, p, b)\right) \bigcup R^{\prime}(n, p) .
$$

By definition, we have that $K^{\prime}(n, p, b), \Sigma K^{\prime}(n, p)$ and $R^{\prime}(n, p)$ coincide with $i_{n, p}(K(n, p, b)), i_{n, p}(\Sigma K(n, p))$ and $i_{n, p}\left(V_{n, p}^{\text {row }}\right)$ respectively.

We prove that $i_{n, p}$ induces a homeomorphism of $K(n, p)$ onto $K^{\prime}(n, p)$. Let $D: \Omega_{\Delta} \rightarrow K(n, p)$ be the map defined as follows. For an element $\alpha=$ $S \Delta(\mathbf{d}) M\left(\begin{array}{l}1 \\ p\end{array}\right) \in \Omega_{\Delta}$, let $b(\alpha)$ denote the real number $d_{p} / d_{p-1}$. Then we set

$$
D(\alpha)=\left[S, M\left(\begin{array}{l}
1 \\
p
\end{array}\right), b(\alpha)\right] \in K(n, p) .
$$

We show that $D$ is well defined. Suppose that $\Delta(\mathbf{d})$ is written as $a_{1} E_{i_{1}} \dot{+} a_{2} E_{i_{2}}$ $\dot{+} \cdots \dot{+} a_{s} E_{i_{s}}$, where $a_{1}, \ldots, a_{s}$ are all distinct. Take another diagonalization $S^{\prime} \Delta(\mathbf{d}) M^{\prime}\left(\begin{array}{l}1 \\ p\end{array}\right)$ of $\alpha$. If $d_{p}>0$, then there exist matrices $G_{j} \in O\left(i_{j}\right)(1 \leq j \leq s)$ such that $S^{\prime}=S\left({ }^{t} G_{1} \dot{+} \cdots \dot{+}^{t} G_{s-1} \dot{+}^{t} G_{s}\right)$ and $M^{\prime}\left(\begin{array}{l}1 \\ p\end{array}\right)=\left(G_{1} \dot{+} \cdots \dot{+} G_{s-1} \dot{+} G_{s}\right)$ $M\left(\begin{array}{l}1 \\ p\end{array}\right)$ by Lemma 5.4. If $d_{p-1}=d_{p}>0$, then $b(\alpha)=1$ and $S M\left(\begin{array}{l}1 \\ p\end{array}\right)=S^{\prime} M^{\prime}\left(\begin{array}{l}1 \\ p\end{array}\right) \in$ $\left(E_{p} \times O(n-p)\right) \backslash O(n)$. If $d_{p-1}>d_{p}>0$, then $i_{s}=1$ and so $G_{s} \in O(1)$. Hence, we have $\left[S, M\left(\begin{array}{l}1 \\ p\end{array}\right), b(\alpha)\right]=\left[S^{\prime}, M^{\prime}\left(\begin{array}{l}1 \\ p\end{array}\right), b(\alpha)\right]$ in $K(n, p)$ by Remark 2.1. If $d_{p}=0$, then by Lemma 5.4 there exist matrices $G_{j} \in O\left(i_{j}\right)$ with $i_{s}=1$ such that $S^{\prime}\left(\begin{array}{l}1 \\ p\end{array}\right)=S\left(\begin{array}{l}1 \\ p\end{array}\right)\left({ }^{t} G_{1} \dot{+} \cdots \dot{+}{ }^{t} G_{s}\right)$ and $M^{\prime}\left(\begin{array}{c}1 \\ p-1\end{array}\right)=\left(G_{1} \dot{+} \cdots \dot{+} G_{s-1}\right) M\left(\begin{array}{c}1 \\ p-1\end{array}\right)$. This implies that $\left[S, M\left(\begin{array}{c}1 \\ p-1\end{array}\right)\right]=\left[S^{\prime}, M^{\prime}\left(\begin{array}{c}1 \\ p-1\end{array}\right)\right]$ in $\Sigma K(n, p)$ by Remark 2.1. Thus $D$ 
is well defined. The fact that $D$ is continuous will be proved in Proposition 6.3 below.

Now we have the following lemma.

\section{Lemma 6.2.}

(1) The map $i_{n, p} \circ D: \Omega_{\Delta} \rightarrow K^{\prime}(n, p)$ is a retraction which maps $\Sigma_{\Delta}$ and $\Omega_{\Delta} \backslash \Sigma_{\Delta}$ onto $\Sigma K^{\prime}(n, p)$ and $K^{\prime}(n, p) \backslash \Sigma K^{\prime}(n, p)$ respectively.

(2) The maps $i_{n, p}: K(n, p) \rightarrow K^{\prime}(n, p)$ and $i_{n, p} \mid \Sigma K(n, p): \Sigma K(n, p) \rightarrow$ $\Sigma K^{\prime}(n, p)$ are homeomorphisms.

Proof. Since $K(n, p)$ is a compact space, it is enough to prove that $D \circ$ $i_{n, p}=i d_{K(n, p)}$ and $i_{n, p} \circ D \mid K^{\prime}(n, p)=i d_{K^{\prime}(n, p)}$ and that the map $i_{n, p} \circ D$ preserves $\Sigma_{\Delta}$ and $\Omega_{\Delta} \backslash \Sigma_{\Delta}$.

Let $\left[S, M\left(\begin{array}{l}1 \\ p\end{array}\right), b\right]$ be an element of $K(n, p)$. Then we have

$$
D \circ i_{n, p}\left(\left[S, M\left(\begin{array}{l}
1 \\
p
\end{array}\right), b\right]\right)=D\left(S \Delta(1, \ldots, 1, b) M\left(\begin{array}{l}
1 \\
p
\end{array}\right)\right)=\left[S, M\left(\begin{array}{l}
1 \\
p
\end{array}\right), b\right] .
$$

On the other hand, let $\alpha=S \Delta(1, \ldots, 1, b) M\left(\begin{array}{l}1 \\ p\end{array}\right) \in K^{\prime}(n, p)$. Then we have

$$
i_{n, p} \circ D(\alpha)=i_{n, p}\left(\left[S, M\left(\begin{array}{l}
1 \\
p
\end{array}\right), b\right]\right)=S \Delta(1, \ldots, 1, b) M\left(\begin{array}{l}
1 \\
p
\end{array}\right)=\alpha .
$$

If $\alpha=S \Delta(\mathbf{d}) M\left(\begin{array}{l}1 \\ p\end{array}\right) \in \Sigma_{\Delta}$, namely $d_{p}=0$, then $b(\alpha)=0$ and $i_{n, p} \circ D(\alpha) \in$ $\Sigma K^{\prime}(n, p)$ and vice versa. This proves the lemma.

Let $r_{\lambda}: \Delta^{p}(1) \rightarrow \Delta^{p}(1)$ be the deformation retraction of $\Delta^{p}(1)$ to $I_{\Delta}^{p}$ defined by

$$
r_{\lambda}\left(1, d_{2}, \ldots, d_{p}\right)=(1-\lambda)\left(1, d_{2}, \ldots, d_{p}\right)+\lambda\left(1, \ldots, 1, d_{p} / d_{p-1}\right) .
$$

We should note that if $d_{i}=d_{j}$, then we have that $r_{\lambda}\left(d_{i}\right)=r_{\lambda}\left(d_{j}\right)$ for $0 \leq \lambda \leq 1$. For an element $\alpha=S \Delta(\mathbf{d}) M\left(\begin{array}{l}1 \\ p\end{array}\right) \in \Omega_{\Delta}$, we define $D_{\lambda}(\alpha)$ by

$$
D_{\lambda}(\alpha)=(1-\lambda) \alpha+\lambda i_{n, p} \circ D(\alpha)=S \Delta\left(r_{\lambda}(\mathbf{d})\right) M\left(\begin{array}{l}
1 \\
p
\end{array}\right) .
$$

Then we have the following proposition.

Proposition 6.3. The homotopy $D_{\lambda}: \Omega_{\Delta} \rightarrow \Omega_{\Delta}$ is a deformation retraction of $\Omega_{\Delta}$ to $K^{\prime}(n, p)$ such that $D_{\lambda}$ preserves $\Sigma_{\Delta}$ and $\Omega_{\Delta} \backslash \Sigma_{\Delta}$ respectively. In particular, $D_{\lambda} \mid \Sigma_{\Delta}$ induces a deformation retraction of $\Sigma_{\Delta}$ to $\Sigma K^{\prime}(n, p)$. 
Proof. We first show that $D(\alpha)$ is continuous. Take a sequence $\left\{\alpha_{k}\right\}$ converging to $\alpha \in \Omega_{\Delta}$. We consider the sequence $\left\{{ }^{t} S \alpha_{k}\left({ }^{t} M\right)\right\}$ in place of $\alpha_{k}$. By (6.2), it is clear that $D\left({ }^{t} S \alpha\left({ }^{t} M\right)\right)={ }^{t} S D(\alpha)\left({ }^{t} M\right)$. Furthermore, $\lim _{k \rightarrow \infty} D\left(\alpha_{k}\right)=D(\alpha)$ holds if and only if $\lim _{k \rightarrow \infty} D\left({ }^{t} S \alpha_{k}\left({ }^{t} M\right)\right)=D\left({ }^{t} S \alpha\right.$ $\left.\times\left({ }^{t} M\right)\right)$ holds. Therefore, it is enough for the continuity to prove the last equality. For this, let $\alpha_{k}=S^{k} \Delta\left(\mathbf{d}^{k}\right) M^{k}\left(\begin{array}{l}1 \\ p\end{array}\right)$ be diagonalizations. We note ${ }^{t} S \alpha\left({ }^{t} M\right)=\left(\Delta(\mathbf{d}), \mathbf{0}_{p \times(n-p)}\right)$. If $d_{p}=0$, then we have $\lim _{k \rightarrow \infty} d_{p}^{k}=0$ by Lemma 5.6.

Considering the expressions ${ }^{t} S S^{k} \Delta\left(\mathbf{d}^{k}\right)\left(M^{k}\left(\begin{array}{l}1 \\ p\end{array}\right)^{t} M\right)$, we have

$$
\lim _{k \rightarrow \infty}{ }^{t} S S^{k} \Delta\left(\mathbf{d}^{k}\right)\left(M^{k}\left(\begin{array}{l}
1 \\
p
\end{array}\right)^{t} M\right)=\left(\Delta(\mathbf{d}), \mathbf{0}_{p \times(n-p)}\right) .
$$

By Lemma 5.6, we have $\delta\left({ }^{t} S S^{k}\right)=\delta\left({ }^{t} S S^{k}\right)_{1} \dot{+} \cdots \dot{+} \delta\left({ }^{t} S S^{k}\right)_{s}$ such that

(1) if $d_{p} \neq 0$, then $\lim _{k \rightarrow \infty} \delta\left({ }^{t} S S^{k}\right) M^{k}\left(\begin{array}{l}1 \\ p\end{array}\right)^{t} M=\lim _{k \rightarrow \infty}\left(E_{p}, \mathbf{0}_{p \times(n-p)}\right)$,

(2) if $d_{p}=0$, then $\lim _{k \rightarrow \infty}\left(\delta\left({ }^{t} S S^{k}\right)_{1} \dot{+} \cdots \dot{+} \delta\left({ }^{t} S S^{k}\right)_{s-1}\right) M^{k}\left({ }_{p-1}\right)^{t} M=$ $\left(E_{p-1}, \mathbf{0}_{(p-1) \times(n-p+1)}\right)$.

Since $i_{n, p}$ is continuous bijection, we have

$$
\begin{aligned}
i_{n, p}\left(\lim _{k \rightarrow \infty} D\left({ }^{t} S \alpha_{k}\left({ }^{t} M\right)\right)\right) & =\lim _{k \rightarrow \infty} i_{n, p} \circ D\left({ }^{t} S \alpha_{k}\left({ }^{t} M\right)\right) \\
& =\lim _{k \rightarrow \infty}{ }^{t} S S^{k} \Delta\left(r_{1}\left(\mathbf{d}^{k}\right)\right)^{t} M\left(\begin{array}{l}
1 \\
p
\end{array}\right){ }^{t} M \\
& =\lim _{k \rightarrow \infty}\left(\delta ( { } ^ { t } S S ^ { k } ) \Delta ( r _ { 1 } ( \mathbf { d } ^ { k } ) ) ^ { t } M \left(\left(_{p}^{1}\right)^{t} M\right.\right. \\
& =\lim _{k \rightarrow \infty}\left(\Delta\left(r_{1}\left(\mathbf{d}^{k}\right)\right) \delta\left({ }^{t} S S^{k}\right){ }^{t} M\left({ }_{p}^{1}\right)^{t} M\right. \\
& =\left(\Delta\left(r_{1}(\mathbf{d})\right)\left(E_{p}, \mathbf{0}_{p \times(n-p)}\right)\right. \\
& =\left(\Delta\left(r_{1}(\mathbf{d})\right), \mathbf{0}_{p \times(n-p)}\right) \\
& =i_{n, p} \circ D\left({ }^{t} S \alpha\left({ }^{t} M\right)\right) .
\end{aligned}
$$

Hence, $D$ is continuous. This yields by $(6.3)$ that $D_{\lambda}(\alpha)$ is continuous with respec to $\alpha$ and $\lambda$.

We next prove that $D_{\lambda}: \Omega_{\Delta} \rightarrow \Omega_{\Delta}$ is a deformation retraction of $\Omega_{\Delta}$ to $K^{\prime}(n, p)$. Since $D_{1}$ coincides with $i_{n, p} \circ D$, the image of $D_{1}$ is $K^{\prime}(n, p)$. We have by Lemma $6.2(1)$ that $D_{\lambda} \mid K^{\prime}(n, p)=i d_{K^{\prime}(n, p)}$ and that $D_{\lambda}$ preserves $\Sigma_{\Delta}$ and $\Omega_{\Delta} \backslash \Sigma_{\Delta}$. Indeed, if $\alpha=S \Delta\left(\mathbf{d}_{b}\right) M\left(\begin{array}{l}1 \\ p\end{array}\right) \in K^{\prime}(n, p)$, then we have $D_{\lambda}(\alpha)=\alpha$, since $r_{\lambda}\left(\mathbf{d}_{b}\right)=\mathbf{d}_{b}$. Furthermore, $d_{p}=0$ in the expression $\alpha=S \Delta(\mathbf{d}) M\left(\begin{array}{l}1 \\ p\end{array}\right)$ if and only if the $p$-th component of $r_{\lambda}(\mathbf{d})$ is also equal to 0 . This completes the proof. 
Proof of Theorem 2.3. We define the homotopy $R_{\lambda}: \Omega^{n-p+1}(n, p) \rightarrow$ $\Omega^{n-p+1}(n, p)$ by

$$
R_{\lambda}= \begin{cases}R_{2 \lambda}^{\prime} & \text { for } \quad 0 \leq \lambda \leq 1 / 2, \\ D_{2 \lambda-1} & \text { for } \quad 1 / 2 \leq \lambda \leq 1\end{cases}
$$

Then the assertion of Theorem 2.4 follows from Lemma 6.1 and Proposition 6.3 .

\section{$\S 7 . \quad$ Homotopy Type of $\Omega^{n-p+1,0}(n, p)$}

For a subspace $C$ in $\mathbf{R}^{p}$, let $\operatorname{pr}(C)$ be the orthogonal projection of $\mathbf{R}^{p}$ onto $C$. Let $V$ be a subspace of $\mathbf{R}^{n}$. Let $C$ be of dimension 1 and $q: S^{2} V \rightarrow C$ be a quadratic form. Then we say that $q$ is a quadratic form with eigen values $\pm a$ if every eigen value of $q$ is equal to either $a$ or $-a$.

We begin by studying the image $\mathcal{I}_{n, p}(\mathcal{K}(n, p, \sigma, b))$. The following observation of this image will be helpful in understanding the arguments in Sections 7 and 8. By definition, it is clear that $\mathcal{I}_{n, p}\left(V_{n, p}^{r o w}\right)=R^{\prime}(n, p) \times \mathbf{0}_{n \times n}^{p}$, where $\mathbf{0}_{n \times n}^{p}$ refers to the null-homomorphism in $\operatorname{Hom}\left(S^{2} \mathbf{R}^{n}, \mathbf{R}^{p}\right), \mathcal{I}_{n, p}(\mathcal{K}(n, p, \sigma, b)) \subset$ $K^{\prime}(n, p, b) \times \operatorname{Hom}\left(S^{2} \mathbf{R}^{n}, \mathbf{R}^{p}\right)$ and $\mathcal{I}_{n, p}(\Sigma \mathcal{K}(n, p, \sigma)) \subset \Sigma K^{\prime}(n, p) \times \operatorname{Hom}\left(S^{2} \mathbf{R}^{n}\right.$, $\left.\mathbf{R}^{p}\right)$.

Let $0 \leq b<1$. For an element $\alpha \in K^{\prime}(n, p, b)$ with diagonalization $\alpha=$ $S \Delta\left(\mathbf{d}_{b}\right) M\left(\begin{array}{l}1 \\ p\end{array}\right)$, we denote, by $C_{\alpha}$, the subspace of dimension 1 in $\mathbf{R}^{p}$ generated by $\overline{\mathbf{s}}_{p}$ and by $K_{\alpha}$, the subspace of dimension $n-p+1$ in $\mathbf{R}^{n}$ generated by ${ }^{t} \mathbf{m}_{p}, \ldots,{ }^{t} \mathbf{m}_{n}$ respectively. Since $b<1$, it follows from Lemma 5.4 that $C_{\alpha}$ and $K_{\alpha}$ are independently defined from the choice of a diagonalization. Let $K_{\alpha}^{\perp}$ and $C_{\alpha}^{\perp}$ be the orthogonal complements of $K_{\alpha}$ in $\mathbf{R}^{n}$ and of $C_{\alpha}$ in $\mathbf{R}^{p}$ respectively. If $0<b<1$, then we have that $\alpha^{-1}\left(C_{\alpha}\right)=K_{\alpha}$, and the orthogonal complement of $\operatorname{Ker}(\alpha)$ in $K_{\alpha}$ is generated by the vector ${ }^{t} \mathbf{m}_{p}$, which is invariantly determined by $\alpha$. If $b=0$, then $K_{\alpha}$ coincides with $\operatorname{Ker}(\alpha)$ and $C_{\alpha}$ is identified with $\mathbf{R}^{p} / \operatorname{Im}(\alpha)$ through the canonical isomorphism $C_{\alpha} \subset \mathbf{R}^{p} \stackrel{\text { projection }}{\longrightarrow} \mathbf{R}^{p} / \operatorname{Im}(\alpha)$.

Let $(\alpha, \beta)$ be an element of $K^{\prime}(n, p, b) \times \operatorname{Hom}\left(S^{2} \mathbf{R}^{n}, \mathbf{R}^{p}\right)$. Let $\beta_{\alpha}$ be the quadratic form defined by $\beta_{\alpha}=\operatorname{pr}\left(\operatorname{Im}(\alpha)^{\perp}\right) \circ\left(\beta \mid S^{2} K_{\alpha}\right)$ as in (1.1). We define the spaces $\mathcal{K}^{\prime}(n, p, \sigma, b)$ for any $b$ with $0<b<1$ and $\Sigma \mathcal{K}^{\prime}(n, p, \sigma)$ for $b=0$ to be the subspaces of $K^{\prime}(n, p, b) \times \operatorname{Hom}\left(S^{2} \mathbf{R}^{n}, \mathbf{R}^{p}\right)$ and $\Sigma K^{\prime}(n, p) \times \operatorname{Hom}\left(S^{2} \mathbf{R}^{n}, \mathbf{R}^{p}\right)$ consisting of all elements $(\alpha, \beta)$ such that

(C-1) $\beta \mid S^{2}\left(\mathbf{R}^{n} \bigcirc K_{\alpha}^{\perp}\right)$ and $\operatorname{pr}\left(C_{\alpha}^{\perp}\right) \circ \beta$ vanish,

(C-2) $\beta_{\alpha}$ is a non-singular quadratic form with eigen values $\pm \sqrt{1-b^{2}}$, 
(C-3) $\beta_{\alpha}$ has the signature $\pm \sigma$,

respectively. For $b=1$, we set $\mathcal{R}^{\prime}(n, p)=R^{\prime}(n, p) \times \mathbf{0}_{n \times n}^{p}$. We define $\mathcal{K}^{\prime}(n, p, \sigma)$, $\mathcal{K}^{\prime}(n, p)$ and $\Sigma \mathcal{K}^{\prime}(n, p)$ to be the union

$$
\begin{aligned}
\mathcal{K}^{\prime}(n, p, \sigma) & =\Sigma \mathcal{K}^{\prime}(n, p) \bigcup\left(\cup_{b \in(0,1)} \mathcal{K}^{\prime}(n, p, \sigma, b)\right) \bigcup \mathcal{R}^{\prime}(n, p), \\
\mathcal{K}^{\prime}(n, p) & =\bigcup_{d=0}^{[(n-p+1) / 2]} \mathcal{K}^{\prime}(n, p, n-p+1-2 d), \\
\Sigma \mathcal{K}^{\prime}(n, p) & =\bigcup_{d=0}^{[(n-p+1) / 2]} \Sigma \mathcal{K}^{\prime}(n, p, n-p+1-2 d),
\end{aligned}
$$

respectively. We first prove that the map $\mathcal{I}_{n, p}$ induces a homeomorphism of $\mathcal{K}(n, p)$ onto $\mathcal{K}^{\prime}(n, p)$.

Theorem 7.1. Let $\sigma$ be a signature as above. Then $\mathcal{I}_{n, p} \mid \mathcal{K}(n, p, \sigma, b)$ for $0<b<1, \mathcal{I}_{n, p} \mid \Sigma \mathcal{K}(n, p, \sigma)$ and $\mathcal{I}_{n, p} \mid V_{n, p}^{\text {row }}$ are topological embeddings of $\mathcal{K}(n, p, \sigma, b)$ onto $\mathcal{K}^{\prime}(n, p, \sigma, b)$, of $\Sigma \mathcal{K}(n, p, \sigma)$ onto $\Sigma \mathcal{K}^{\prime}(n, p, \sigma)$, and of $V_{n, p}^{\text {row }}$ onto $\mathcal{R}^{\prime}(n, p)$ respectively.

Proof. The assertion for $\mathcal{I}_{n, p} \mid V_{n, p}^{\text {row }}$ follows from the fact that the map $\mathcal{I}_{n, p} \mid V_{n, p}^{\text {row }}$ coincides with the composition of the map $i_{n, p}$ and the inclusion $\mathcal{R}^{\prime}(n, p) \subset R^{\prime}(n, p) \times \operatorname{Hom}\left(S^{2} \mathbf{R}^{n}, \mathbf{R}^{p}\right)$.

Let $0<b<1$. Let $[\mathbf{z}]$ be $[S, T, M, \sigma, b]$. By the definition (2.18) of $\alpha([\mathbf{z}])$, it is clear that $\alpha([\mathbf{z}])=S \Delta\left(\mathbf{d}_{b}\right) M\left(\begin{array}{l}1 \\ p\end{array}\right) \in K^{\prime}(n, p, b)$. By the definition (2.18) of $\beta([\mathbf{z}])$ it follows that $\beta([\mathbf{z}]) \mid S^{2}\left(\mathbf{R}^{n} \bigcirc K_{\alpha}^{\perp}\right)$ vanishes, since $K_{\alpha}^{\perp}$ is generated by ${ }^{t} \mathbf{m}_{1}, \ldots,{ }^{t} \mathbf{m}_{p-1}$. Furthermore, $\operatorname{pr}\left(C_{\alpha}^{\perp}\right) \circ \beta([\mathbf{z}])$ vanishes, since $\operatorname{Im} \beta([\mathbf{z}]) \subset C_{\alpha}$. If $\sigma>0$, then the vectors ${ }^{t} \mathbf{m}_{p}$ and $\overline{\mathbf{s}}_{p}$ are determined by Remark 2.4 Case (i) and $\beta([\mathbf{z}])_{\alpha([\mathbf{z}])}$ is a non-singular quadratic form with index $d$ and eigen values $\sqrt{1-b^{2}}$ by (2.18). If $\sigma=0$, then the pair of the vectors $\left({ }^{t} \mathbf{m}_{p}, \overline{\mathbf{s}}_{p}\right)$ are determined up to sign by Remark 2.4 Case (iii) and $\beta([\mathbf{z}])_{\alpha([\mathbf{z}])}$ is a non-singular quadratic form with index $(n-p+1) / 2$ and eigen values $\pm \sqrt{1-b^{2}}$. Hence, $\mathcal{I}_{n, p}([\mathbf{z}])$ lies in $\mathcal{K}^{\prime}(n, p, \sigma, b)$. It is similar to prove that $\operatorname{Im}\left(\mathcal{I}_{n, p} \mid \Sigma \mathcal{K}(n, p, \sigma)\right) \subset$ $\Sigma \mathcal{K}^{\prime}(n, p, \sigma)$.

We show the surjectivity. Let $(\alpha, \beta)$ be an element of $\mathcal{K}^{\prime}(n, p, \sigma, b)$ or $\Sigma \mathcal{K}^{\prime}(n, p, \sigma)$. In a diagonalization $\alpha=S \Delta\left(\mathbf{d}_{b}\right) M\left(\begin{array}{l}1 \\ p\end{array}\right)$, we have seen that $K_{\alpha}$ and $C_{\alpha}$ have the orthonormal basis ${ }^{t} \mathbf{m}_{p}, \ldots,{ }^{t} \mathbf{m}_{n}$ and $\overline{\mathbf{s}}_{p}$ respectively. With these basis there is a $(n-p+1) \times(n-p+1)$ matrix $B=\left(b_{i j}\right)(p \leq i, j \leq n)$ defined by

$$
\beta_{\alpha}\left({ }^{t} \mathbf{m}_{i},{ }^{t} \mathbf{m}_{j}\right)=\operatorname{pr}\left(C_{\alpha}\right) \circ \beta\left({ }^{t} \mathbf{m}_{i},{ }^{t} \mathbf{m}_{j}\right)=b_{i j} \overline{\mathbf{s}}_{p} .
$$


By the properties (C-1) to (C-3), $B$ is symmetric and non-singular of signature $\pm(c-d)$ with eigen values $\pm \sqrt{1-b^{2}}$. Suppose that $B$ has the signature $\delta(c-d)$ with $\delta= \pm 1$. Then there exists a matrix $T \in O(n-p+1)$ such that

$$
T B^{t} T=\delta \sqrt{1-b^{2}}\left(E_{c} \dot{+}\left(-E_{d}\right)\right)
$$

with $c \geq d$. Hence, we have

$$
\beta_{\alpha}\left({ }^{t} \mathbf{m}_{i},{ }^{t} \mathbf{m}_{j}\right)=\sqrt{1-b^{2}}\left\{{ }^{t} \mathbf{m}_{i}{ }^{t} M\left(\begin{array}{l}
p \\
n
\end{array}\right)^{t} T\left(E_{c} \dot{+}\left(-E_{d}\right)\right) T M\left(\begin{array}{l}
p \\
n
\end{array}\right) \mathbf{m}_{j}\right\}\left(\delta \overline{\mathbf{s}}_{p}\right) .
$$

This induces

$$
\beta_{\alpha}(\mathbf{x}, \mathbf{y})=\sqrt{1-b^{2}}\left\{{ }^{t} \mathbf{x}^{t} M\left(\begin{array}{l}
p \\
n
\end{array}\right)^{t} T\left(E_{c} \dot{+}\left(-E_{d}\right)\right) T M\left(\begin{array}{l}
p \\
n
\end{array}\right) \mathbf{y}\right\}\left(\delta \overline{\mathbf{s}}_{p}\right) .
$$

Let $b>0$. If we set $S^{\prime}=S\left(E_{p-1} \dot{+}(\delta)\right)$ and $M^{\prime}=\left(E_{p-1} \dot{+}(\delta) \dot{+} E_{n-p}\right) M$, then we have that $\beta_{\alpha}(\mathbf{x}, \mathbf{y})$ coincides with

$$
\beta\left(\left[S^{\prime}, T^{\prime}, M^{\prime}, \sigma, b\right]\right)(\mathbf{x}, \mathbf{y})=\sqrt{1-b^{2}}\left\{{ }^{t} \mathbf{x}^{t} M^{\prime}\left(\begin{array}{l}
p \\
n
\end{array}\right)^{t} T\left(E_{c} \dot{+}\left(-E_{d}\right)\right) T M^{\prime}\left(\begin{array}{l}
p \\
n
\end{array}\right) \mathbf{y}\right\} \overline{\mathbf{s}}_{p}^{\prime} .
$$

Since $\alpha=S \Delta\left(\mathbf{d}_{b}\right) M\left(\begin{array}{l}1 \\ p\end{array}\right)=S^{\prime} \Delta\left(\mathbf{d}_{b}\right) M^{\prime}\left(\begin{array}{l}1 \\ p\end{array}\right)$ in $K(n, p, b)$, we have that $\alpha([S, T, M, \sigma, b])=\alpha\left(\left[S^{\prime}, T^{\prime}, M^{\prime}, \sigma, b\right]\right)$. Thus we concludes $\mathcal{I}_{n, p}\left(\left[S^{\prime}, T^{\prime}, M^{\prime}, \sigma\right.\right.$, $b])=(\alpha, \beta)$.

Let $b=0$. If we set $S^{\prime}=S\left(E_{p-1} \dot{+}(\delta)\right)$ and $M^{\prime}=\left(E_{p-1} \dot{+} T\right) M$, then we have that $\beta(\mathbf{x}, \mathbf{y})$ coincides with

$$
\beta\left(\left[S^{\prime}, M^{\prime}, \sigma\right]\right)(\mathbf{x}, \mathbf{y})=\left\{{ }^{t} \mathbf{x}^{t} M^{\prime}\left(\begin{array}{l}
p \\
n
\end{array}\right)\left(E_{c}+\left(-E_{d}\right)\right) M^{\prime}\left(\begin{array}{l}
p \\
n
\end{array}\right) \mathbf{y}\right\} \overline{\mathbf{s}}_{p}^{\prime} .
$$

Since $M\left(\begin{array}{c}1 \\ p-1\end{array}\right)=M^{\prime}\left(\begin{array}{c}1 \\ p-1\end{array}\right)$ and $\alpha=S \Delta\left(\mathbf{d}_{0}\right) M\left(\begin{array}{l}1 \\ p\end{array}\right)=S^{\prime} \Delta\left(\mathbf{d}_{0}\right) M^{\prime}\left(\begin{array}{l}1 \\ p\end{array}\right)$ in $\Sigma K(n, p)$, we have that $\alpha([S, M, \sigma])=\alpha\left(\left[S^{\prime}, M^{\prime}, \sigma\right]\right)$. Thus we concludes $\mathcal{I}_{n, p}\left(\left[S^{\prime}, M^{\prime}, \sigma\right]\right)$ $=(\alpha, \beta)$.

It remains to prove the injectivity. Let $[\mathbf{z}]=[S, T, M, \sigma, b],\left[\mathbf{z}^{\prime}\right]=\left[S^{\prime}, T^{\prime}\right.$, $\left.M^{\prime}, \sigma, b\right]$ in $\mathcal{K}^{\prime}(n, p, \sigma, b)$, or $[\mathbf{z}]=[S, M, \sigma]$ and $\left[\mathbf{z}^{\prime}\right]=\left[S^{\prime}, M^{\prime}, \sigma\right]$ in $\Sigma \mathcal{K}^{\prime}(n, p, \sigma)$ respectively. Suppose that $\mathcal{I}_{n, p}([\mathbf{z}])=\mathcal{I}_{n, p}\left(\left[\mathbf{z}^{\prime}\right]\right)$. This implies that

$$
\alpha=S \Delta\left(\mathbf{d}_{b}\right) M\left(\begin{array}{l}
1 \\
p
\end{array}\right)=S^{\prime} \Delta\left(\mathbf{d}_{b}\right) M^{\prime}\left(\begin{array}{l}
1 \\
p
\end{array}\right),
$$

and for $\mathbf{x}, \mathbf{y} \in \mathbf{R}^{n}$,

$$
\begin{aligned}
& \sqrt{1-b^{2}}\left\{{ }^{t} \mathbf{x}^{t} M\left(\begin{array}{l}
p \\
n
\end{array}\right)^{t} T\left(E_{c} \dot{+}\left(-E_{d}\right)\right) T M\left(\begin{array}{l}
p \\
n
\end{array}\right) \mathbf{y}\right\} \overline{\mathbf{s}}_{p} \\
& \quad=\sqrt{1-b^{2}}\left\{{ }^{t} \mathbf{x}^{t} M^{\prime}\left(\begin{array}{l}
p \\
n
\end{array}\right){ }^{t} T^{\prime}\left(E_{c} \dot{+}\left(-E_{d}\right)\right) T^{\prime} M^{\prime}\left(\begin{array}{l}
p \\
n
\end{array}\right) \mathbf{y}\right\} \overline{\mathbf{s}}_{p}^{\prime},
\end{aligned}
$$

where if $b=0$, then $T=T^{\prime}=E_{n-p+1}$. For $b<1$ we need deal with the following four cases. 
Case (i): $\sigma>0$ and $0<b<1$. By (7.2) and Lemma 5.4 there exist $G \in O(p-1)$ and $(\delta) \in O(1)$ such that $S^{\prime}=S\left({ }^{t} G \dot{+}(\delta)\right)$ and $M^{\prime}\left(\begin{array}{l}1 \\ p\end{array}\right)=$ $(G \dot{+}(\delta)) M\left(\begin{array}{l}1 \\ p\end{array}\right)$. In this case a unit basis of $C_{\alpha}$ is uniquely selected so that $\beta_{\alpha}$ has the index $d$, and hence we have $\overline{\mathbf{s}}_{p}=\overline{\mathbf{s}}_{p}^{\prime}$, namely $\delta=1$ by (7.3). Since $\alpha\left({ }^{t} \mathbf{m}_{p}\right)=S\left(b \mathbf{e}_{p}\right)=b \overline{\mathbf{s}}_{p}$ and $\alpha\left({ }^{t} \mathbf{m}_{p}^{\prime}\right)=S^{\prime}\left(b \mathbf{e}_{p}\right)=b \overline{\mathbf{s}}_{p}^{\prime}$ and $b>0$, we have $\mathbf{m}_{p}=\mathbf{m}_{p}^{\prime}$. Furthermore, it follows from (7.3) and Lemma 5.5 that there exist matrices $T_{1} \in O(c)$ and $T_{2} \in O(d)$ such that $T^{\prime} M^{\prime}\left(\begin{array}{l}p \\ n\end{array}\right)=\left(T_{1} \dot{+} T_{2}\right) T M\left(\begin{array}{l}p \\ n\end{array}\right)$. This induces $M^{\prime}\left(\begin{array}{l}p \\ n\end{array}\right)={ }^{t} T^{\prime}\left(T_{1} \dot{+} T_{2}\right) T M\left(\begin{array}{l}p \\ n\end{array}\right)$. Setting $L^{\prime}={ }^{t} T^{\prime}\left(T_{1}+T_{2}\right) T$, we have that $T^{\prime}=\left(T_{1}+T_{2}\right) T^{t} L^{\prime}$ and $M^{\prime}\left(\begin{array}{l}p \\ n\end{array}\right)=L^{\prime} M\left(\begin{array}{l}p \\ n\end{array}\right)$. Since $\mathbf{m}_{p}=\mathbf{m}_{p}^{\prime}$, we have $L^{\prime}=((1)+L)$ for some $L \in O(n-p)$. This implies

$$
\begin{aligned}
{\left[S^{\prime}, T^{\prime}, M^{\prime}, \sigma, b\right] } & =\left[S\left({ }^{t} G \dot{+}(1)\right),\left(T_{1} \dot{+} T_{2}\right) T\left((1) \dot{+}{ }^{t} L\right),(G \dot{+}(1) \dot{+} L) M, \sigma, b\right] \\
& =[S, T, M, \sigma, b]
\end{aligned}
$$

in $\mathcal{K}(n, p, \sigma, b)$ by Remark 2.4 Case (i).

Case (ii): $\sigma>0$ and $b=0$. By (7.2) and Lemma 5.4 there exist $G \in$ $O(p-1)$ and $(\delta) \in O(1)$ such that $S^{\prime}=S\left({ }^{t} G \dot{+}(\delta)\right)$ and $M^{\prime}\left(\begin{array}{c}1 \\ p-1\end{array}\right)=G M\left(\begin{array}{c}1 \\ p-1\end{array}\right)$. By (7.3) and Lemma 5.5 there exist matrices $T_{1} \in O(c)$ and $T_{2} \in O(d)$ such that $M^{\prime}\left(\begin{array}{l}p \\ n\end{array}\right)=\left(T_{1}+T_{2}\right) M\left(\begin{array}{l}p \\ n\end{array}\right)$. This implies

$$
\left[S^{\prime}, M^{\prime}, \sigma\right]=\left[S\left({ }^{t} G \dot{+}(\delta)\right),\left(G \dot{+} T_{1} \dot{+} T_{2}\right) M, \sigma\right]=[S, M, \sigma]
$$

in $\Sigma \mathcal{K}(n, p, \sigma)$ by Remark 2.4 Case (ii).

Case (iii): $\sigma=0$ and $0<b<1$. By (7.2) and Lemma 5.4, there exist $G \in O(p-1)$ and $(\delta) \in O(1)$ such that $S^{\prime}=S\left({ }^{t} G \dot{+}(\delta)\right)$ and $M^{\prime}\left(\begin{array}{l}1 \\ p\end{array}\right)=$ $(G \dot{+}(\delta)) M\left(\begin{array}{l}1 \\ p\end{array}\right)$. In this case we have $\overline{\mathbf{s}}_{p}=\delta \overline{\mathbf{s}}_{p}^{\prime}$ and $\mathbf{m}_{p}=\delta \mathbf{m}_{p}^{\prime}$. If $\delta=1$, then, by (7.3) and Lemma 5.5, there exist matrices $T_{1}, T_{2} \in O(c)$ such that $T^{\prime} M^{\prime}\left(\begin{array}{l}p \\ n\end{array}\right)=\left(T_{1}+T_{2}\right) T M\left(\begin{array}{l}p \\ n\end{array}\right)$. This induces $M^{\prime}\left(\begin{array}{l}p \\ n\end{array}\right)={ }^{t} T^{\prime}\left(T_{1}+T_{2}\right) T M\left(\begin{array}{l}p \\ n\end{array}\right)$. Setting $L^{\prime}={ }^{t} T^{\prime}\left(T_{1} \dot{+} T_{2}\right) T$, we have $T^{\prime}=\left(T_{1} \dot{+} T_{2}\right) T^{t} L^{\prime}$ and $M^{\prime}\left(\begin{array}{l}p \\ n\end{array}\right)=L^{\prime} M\left(\begin{array}{l}p \\ n\end{array}\right)$. Since $\mathbf{m}_{p}=\mathbf{m}_{p}^{\prime}$, we have $L^{\prime}=((1) \dot{+} L)$ for some $L \in O(n-p)$. This implies $\left[S^{\prime}, T^{\prime}, M^{\prime}, 0, b\right]=[S, T, M, 0, b]$ in $\mathcal{K}(n, p, 0, b)$ as in the Case (i). If $\delta=-1$, then we have $\overline{\mathbf{s}}_{p}=-\overline{\mathbf{s}}_{p}^{\prime}$ and $\mathbf{m}_{p}=-\mathbf{m}_{p}^{\prime}$. By (7.2) and Lemma 5.5 it follows that

$$
\begin{aligned}
& { }^{t} M^{\prime}\left(\begin{array}{l}
p \\
n
\end{array}\right)^{t} T^{\prime}\left(E_{c} \dot{+}\left(-E_{c}\right)\right) T^{\prime} M^{\prime}\left(\begin{array}{l}
p \\
n
\end{array}\right) \\
& ={ }^{t} M\left(\begin{array}{l}
p \\
n
\end{array}\right)^{t} T\left(\left(-E_{c}\right) \dot{+} E_{c}\right) T M\left(\begin{array}{l}
p \\
n
\end{array}\right) \\
& ={ }^{t} M\left(\begin{array}{l}
p \\
n
\end{array}\right)^{t} T\left(\begin{array}{ll}
0 & E_{c} \\
E_{c} & 0
\end{array}\right)\left(E_{c} \dot{+}\left(-E_{c}\right)\right)\left(\begin{array}{ll}
0 & E_{c} \\
E_{c} & 0
\end{array}\right) T M\left(\begin{array}{l}
p \\
n
\end{array}\right) .
\end{aligned}
$$


By Lemma 5.5 there exist matrices $T_{1} \in O(c)$ and $T_{2} \in O(c)$ such that

$$
T^{\prime} M^{\prime}\left(\begin{array}{l}
p \\
n
\end{array}\right)=\left(\begin{array}{ll}
0 & E_{c} \\
E_{c} & 0
\end{array}\right)\left(T_{1}+T_{2}\right) T M\left(\begin{array}{l}
p \\
n
\end{array}\right) .
$$

Hence, we have

$$
M^{\prime}\left(\begin{array}{l}
p \\
n
\end{array}\right)={ }^{t} T^{\prime}\left(\begin{array}{ll}
0 & E_{c} \\
E_{c} & 0
\end{array}\right)\left(T_{1}+T_{2}\right) T M\left(\begin{array}{l}
p \\
n
\end{array}\right) .
$$

Setting

$$
L^{\prime}={ }^{t} T^{\prime}\left(\begin{array}{ll}
0 & E_{c} \\
E_{c} & 0
\end{array}\right)\left(T_{1}+T_{2}\right) T,
$$

we have

$$
T^{\prime}=\left(\begin{array}{ll}
0 & E_{c} \\
E_{c} & 0
\end{array}\right)\left(T_{1}+T_{2}\right) T^{t} L^{\prime}
$$

and $M^{\prime}\left(\begin{array}{l}p \\ n\end{array}\right)=L^{\prime} M\left(\begin{array}{l}p \\ n\end{array}\right)$. Since $\mathbf{m}_{p}=-\mathbf{m}_{p}^{\prime}$, we have $L^{\prime}=((-1) \dot{+} L)$ for some $L \in O(n-p)$. This implies

$$
\begin{aligned}
& {\left[S^{\prime}, T^{\prime}, M^{\prime}, 0, b\right]} \\
& =\left[S\left({ }^{t} G \dot{+}(-1)\right),\left(\begin{array}{ll}
0 & E_{c} \\
E_{c} & 0
\end{array}\right)\left(T_{1} \dot{+} T_{2}\right) T\left((-1) \dot{+}{ }^{t} L\right),(G \dot{+}(-1) \dot{+} L) M, 0, b\right] \\
& =((-1), L) \cdot\left[S\left({ }^{t} G \dot{+}(1)\right),\left(T_{1} \dot{+} T_{2}\right) T,\left(G \dot{+} E_{n-p+1}\right) M, 0, b\right] \\
& =((-1), L) \cdot[S, T, M, 0, b] \\
& =[S, T, M, 0, b]
\end{aligned}
$$

in $\mathcal{K}(n, p, 0, b)$ by Remark 2.4 Case (iii).

Case (iv): $\sigma=0$ and $b=0$. By (7.2) and Lemma 5.4 there exist $G \in$ $O(p-1)$ and $(\delta) \in O(1)$ such that $S^{\prime}=S\left({ }^{t} G \dot{+}(\delta)\right)$ and $M^{\prime}\left(\begin{array}{c}1 \\ p-1\end{array}\right)=G M\left(\begin{array}{c}1 \\ p-1\end{array}\right)$. Since $b=0$, we have $\operatorname{Ker}(\alpha)=\left\{{ }^{t} \mathbf{m}_{p}, \ldots,{ }^{t} \mathbf{m}_{n}\right\}=\left\{{ }^{t} \mathbf{m}_{p}^{\prime}, \ldots,{ }^{t} \mathbf{m}_{n}^{\prime}\right\}$. If $\delta=1$, then $\overline{\mathbf{s}}_{p}=\overline{\mathbf{s}}_{p}^{\prime}$. By (7.3) and Lemma 5.5 we have matrices $T_{1}, T_{2} \in O(c)$ such that $M^{\prime}\left(\begin{array}{l}p \\ n\end{array}\right)=\left(T_{1}+T_{2}\right) M\left(\begin{array}{l}p \\ n\end{array}\right)$. This gives

$$
\left[S^{\prime}, M^{\prime}, 0\right]=\left[S\left({ }^{t} G \dot{+}(1)\right),\left(G \dot{+} T_{1} \dot{+} T_{2}\right) M, 0\right]=[S, M, 0]
$$

in $\Sigma \mathcal{K}(n, p, 0)$ by Remark 2.4 Case (iv). If $\delta=-1$, then $\overline{\mathbf{s}}_{p}=-\overline{\mathbf{s}}_{p}^{\prime}$. By using Lemma 5.5 similarly as in the Case (iii), we can show that there exist matrices 
$T_{1}, T_{2} \in O(c)$ such that

$$
M^{\prime}\left(\begin{array}{l}
p \\
n
\end{array}\right)=\left(\begin{array}{ll}
0 & E_{c} \\
E_{c} & 0
\end{array}\right)\left(T_{1}+T_{2}\right) M\left(\begin{array}{l}
p \\
n
\end{array}\right) .
$$

Hence, we have

$$
\begin{aligned}
{\left[S^{\prime}, M^{\prime}, 0\right] } & =\left[S\left({ }^{t} G \dot{+}(-1)\right),\left(G \dot{+}\left(\begin{array}{ll}
0 & E_{c} \\
E_{c} & 0
\end{array}\right)\left(T_{1} \dot{+} T_{2}\right)\right) M, 0\right] \\
& =(-1) \cdot\left[S\left({ }^{t} G \dot{+}(1)\right),\left(G \dot{+} T_{1} \dot{+} T_{2}\right) M, 0\right] \\
& =(-1) \cdot[S, M, 0] \\
& =[S, M, 0]
\end{aligned}
$$

in $\Sigma \mathcal{K}(n, p, 0)$ by Remark 2.4 Case (iv).

This completes the proof.

§8. Deformation Retraction of $\Omega^{n-p+1,0}(n, p)$ to $\mathcal{K}^{\prime}(n, p)$

In this section we complete the proof of Theorem 2.6. Let $C=\left(c_{i j}\right)(1 \leq$ $i, j \leq n)$ be an $n \times n$ matrix. The norm $\|C\|$ is defined to be $\left(\sum_{i=1}^{n} \sum_{j=1}^{n} c_{i j}^{2}\right)^{1 / 2}$. If $L, U \in O(n)$, then we have $\|L C U\|=\|C\|$. We canonically identify an element $\beta \in \operatorname{Hom}\left(S^{2} \mathbf{R}^{n}, \mathbf{R}^{p}\right)$ with the $p$-tuple $\left(C_{1}, \ldots, C_{p}\right)$ of symmetric $n \times n$ matrices. Then the norm $\|\beta\|$ is defined to be $\left(\sum_{i=1}^{p}\left\|C_{i}\right\|^{2}\right)^{1 / 2}$. In particular, we have

$$
\begin{aligned}
\|\beta([S, T, M, \sigma, b])\| & =\sqrt{1-b^{2}}\left\|^{t} M\left(\begin{array}{l}
p \\
n
\end{array}\right)^{t} T\left(E_{c} \dot{+}\left(-E_{d}\right)\right) T M\left(\begin{array}{l}
p \\
n
\end{array}\right)\right\| \\
& =\sqrt{1-b^{2}}\left\|^{t} M\left(\mathbf{0}_{(p-1) \times(p-1)} \dot{+}{ }^{t} T\left(E_{c} \dot{+}\left(-E_{d}\right)\right) T\right) M\right\| \\
& =\sqrt{1-b^{2}}\left\|\left(\mathbf{0}_{(p-1) \times(p-1)} \dot{+}{ }^{t} T\left(E_{c} \dot{+}\left(-E_{d}\right)\right) T\right)\right\| \\
& =\sqrt{1-b^{2}}\left\|\left(E_{c} \dot{+}\left(-E_{d}\right)\right)\right\| \\
& =\sqrt{\left(1-b^{2}\right)(n-p+1)} .
\end{aligned}
$$

If an element $\alpha \in K^{\prime}(n, p)$ is written as $S \Delta\left(\mathbf{d}_{b}\right) M\left(\begin{array}{l}1 \\ p\end{array}\right)$, then we define the continuous functions $b(\alpha)$ and $\|x(\alpha)\|$ to be $b$ and $\sqrt{\left(1-b(\alpha)^{2}\right)(n-p+1)}$ respectively.

Proof of Theorem 2.6. Using the deformation retraction $R_{\lambda}$ of $\Omega^{n-p+1}$ $\times(n, p)$ to $K^{\prime}(n, p)$ in Theorem 2.3, we first define a deformation retraction $H_{\lambda}$ of $\Omega^{n-p+1,0}(n, p)$ to $\left(\pi_{1}^{2} \mid \Omega^{n-p+1,0}(n, p)\right)^{-1}\left(K^{\prime}(n, p)\right)$ by $H_{\lambda}(\alpha, \beta)=\left(R_{\lambda}(\alpha), \beta\right)$ 
for $0 \leq \lambda \leq 1$. Actually, $H_{\lambda}(\alpha, \beta)$ lies in $\Omega^{n-p+1,0}(n, p)$. For, if $\alpha \in \Sigma^{n-p}(n, p)$, then $b(\alpha)>0$, namely $R_{\lambda}(\alpha) \in \Sigma^{n-p}(n, p)$ by Theorem 2.3. If $(\alpha, \beta) \in$ $\Sigma^{n-p+1,0}(n, p)$, namely $b(\alpha)=0$, then $\operatorname{Ker}\left(R_{\lambda}(\alpha)\right)=\operatorname{Ker}(\alpha)$ and $\operatorname{Cok}\left(R_{\lambda}(\alpha)\right)$ $=\operatorname{Cok}(\alpha)$ for any $\lambda$ by (6.1) and (6.3), and hence $\beta_{R_{\lambda}(\alpha)}$ coincides with $\beta_{\alpha}$ for any $\lambda$ by (1.1). This implies $H_{\lambda}(\alpha, \beta) \in \Sigma^{n-p+1,0}(n, p)$. If $\alpha \in K^{\prime}(n, p)$, then $H_{\lambda}(\alpha, \beta)=(\alpha, \beta)$ for $0 \leq \lambda \leq 1$, since $R_{\lambda}(\alpha)=\alpha$. The image of $H_{1}$ clearly coincides with $\left(\pi_{1}^{2} \mid \Omega^{n-p+1,0}(n, p)\right)^{-1}\left(K^{\prime}(n, p)\right)$.

Next let

$$
h_{\lambda}:\left(\pi_{1}^{2} \mid \Omega^{n-p+1,0}(n, p)\right)^{-1}\left(K^{\prime}(n, p)\right) \rightarrow\left(\pi_{1}^{2} \mid \Omega^{n-p+1,0}(n, p)\right)^{-1}\left(K^{\prime}(n, p)\right)
$$

be the homotopy defined by

$$
h_{\lambda}(\alpha, \beta)=\left\{\begin{array}{cl}
\left(\alpha,((1-\lambda)+\lambda\|x(\alpha)\|)(\|\beta\|-2\|x(\alpha)\|) \frac{\beta}{\|\beta\|}+2\|x(\alpha)\| \frac{\beta}{\|\beta\|},\right. \\
\text { if }\|\beta\| \geq 2\|x(\alpha)\| \text { and }\|\beta\| \neq 0, \\
(\alpha, \beta) & \text { if }\|\beta\| \leq 2\|x(\alpha)\| .
\end{array}\right.
$$

Then the image of $h_{1}$ coincides with the union

$$
\left(\pi_{1}^{2} \mid \Omega^{n-p+1,0}(n, p)\right)^{-1}\left(K^{\prime}(n, p) \backslash R^{\prime}(n, p)\right) \bigcup R^{\prime}(n, p) \times \mathbf{0}_{n \times n}^{p} .
$$

If $(\alpha, \beta) \in \mathcal{K}^{\prime}(n, p)$, then we have $\|\beta\|=\sqrt{\left(1-b(\alpha)^{2}\right)(n-p+1)} \leq 2\|x(\alpha)\|$, and hence $h_{\lambda}(\alpha, \beta)=(\alpha, \beta)$ by the definition of $h_{\lambda}$. It is clear that $h_{0}$ is the identity. On the other hand, by Proposition 8.1 below we have a deformation retraction $\mathcal{D}_{\lambda}$ of $\operatorname{Im}\left(h_{1}\right)$ to $\mathcal{K}^{\prime}(n, p)$. Thus we obtain a deformation retraction $\mathcal{R}_{\lambda}$ of $\Omega^{n-p+1,0}(n, p)$ to $K^{\prime}(n, p)$ defined by

$$
\mathcal{R}_{\lambda}(\alpha, \beta)= \begin{cases}H_{3 \lambda}(\alpha, \beta) & 0 \leq \lambda \leq 1 / 3 \\ h_{3 \lambda-1}(\alpha, \beta) & 1 / 3 \leq \lambda \leq 2 / 3 \\ \mathcal{D}_{3 \lambda-2}(\alpha, \beta) & 2 / 3 \leq \lambda \leq 1\end{cases}
$$

This is what we want to prove.

Proposition 8.1. $\quad$ There exists a deformation retraction $\mathcal{D}_{\lambda}$ of $\operatorname{Im}\left(h_{1}\right)$ to $\mathcal{K}^{\prime}(n, p)$ such that $\mathcal{D}_{\lambda}$ preserves $\left(\pi_{1}^{2} \mid \operatorname{Im}\left(h_{1}\right)\right)^{-1}\left(K^{\prime}(n, p) \backslash \Sigma K^{\prime}(n, p)\right)$ and $\left(\pi_{1}^{2} \mid \Sigma^{n-p+1,0}(n, p)\right)^{-1}\left(\Sigma K^{\prime}(n, p)\right)$ respectively. In particular, the restriction $\mathcal{D}_{\lambda} \mid\left(\pi_{1}^{2} \mid \Sigma^{n-p+1,0}(n, p)\right)^{-1}\left(\Sigma K^{\prime}(n, p)\right)$ is a deformation retraction of $\left(\pi_{1}^{2} \mid\right.$ $\left.\Sigma^{n-p+1,0}(n, p)\right)^{-1}\left(\Sigma K^{\prime}(n, p)\right)$ to $\Sigma \mathcal{K}^{\prime}(n, p)$.

The proof of this proposition is rather long. Let $(\alpha, \beta)$ be an element of $\operatorname{Im}\left(h_{1}\right)$. With the basis ${ }^{t} \mathbf{m}_{p}, \ldots,{ }^{t} \mathbf{m}_{n}$ of $K_{\alpha}$ and $\overline{\mathbf{s}}_{p}$ of $C_{\alpha}$, let $B=\left(b_{i j}(\alpha, \beta)\right)$ $(p \leq i, j \leq n)$ be the matrix defined by $\beta_{\alpha}\left({ }^{t} \mathbf{m}_{i},{ }^{t} \mathbf{m}_{j}\right)=b_{i j}(\alpha, \beta) \overline{\mathbf{s}}_{p}$. This 
satisfies that for any $\mathbf{x}, \mathbf{y} \in K_{\alpha}, \beta_{\alpha}(\mathbf{x}, \mathbf{y})=\left\{{ }^{t} \mathbf{x}^{t} M\left(\begin{array}{l}p \\ n\end{array}\right) B M\left(\begin{array}{l}p \\ n\end{array}\right) \mathbf{y}\right\} \overline{\mathbf{s}}_{p}$. Let $a(\alpha, \beta)$ denote the absolute value of $\operatorname{det} B$, which is well defined for $(\alpha, \beta)$. Furthermore, $a(\alpha, \beta)$ is a continuous function. Indeed, it is easy to prove that $a(\alpha, \beta)$ is continuous at $(\alpha, \beta)$ with $b(\alpha)<1$ (use Lemma 8.4 and Corollary 8.5 below if necessary). If $b(\alpha)=1$ and $\left(\alpha^{\prime}, \beta^{\prime}\right)$ converges to $\left(\alpha, \mathbf{0}_{n \times n}^{p}\right)$, then $a\left(\alpha^{\prime}, \beta^{\prime}\right)$ converges to 0 , whatever $\overline{\mathbf{s}}_{p}$ varies. We define the non-negative real number $b(\alpha, \beta)$ by

$$
b(\alpha, \beta)=\frac{b(\alpha)}{\sqrt{a(\alpha, \beta)^{2}+b(\alpha)^{2}}} .
$$

If $b(\alpha)=0$, then $\alpha$ lies in $\Sigma K^{\prime}(n, p)$, and hence $a(\alpha, \beta)$ is not equal to 0 by (C2 ) in Section 7. If $b(\alpha)=1$, then $\beta=\mathbf{0}_{n \times n}^{p}$ and hence, $b(\alpha, \beta)=1$. Therefore, $b(\alpha), a(\alpha, \beta)$ and $b(\alpha, \beta)$ are all continuous functions on $\operatorname{Im}\left(h_{1}\right)$.

We define maps $A: \operatorname{Im}\left(h_{1}\right) \rightarrow K^{\prime}(n, p)$ and $B: \operatorname{Im}\left(h_{1}\right) \rightarrow \operatorname{Hom}\left(S^{2} \mathbf{R}^{n}\right.$, $\left.\mathbf{R}^{p}\right)$, which yields a retraction $\mathcal{D}: \operatorname{Im}\left(h_{1}\right) \rightarrow \mathcal{K}^{\prime}(n, p)$ defined by

$$
\mathcal{D}(\alpha, \beta)=(A(\alpha, \beta), B(\alpha, \beta)) .
$$

Let $(\alpha, \beta)$ be an element of $\operatorname{Im}\left(h_{1}\right)$ with a diagonalization $\alpha=S \Delta\left(\mathbf{d}_{b(\alpha)}\right) M\left(\begin{array}{l}1 \\ p\end{array}\right)$. If $a(\alpha, \beta)=0$, then define $A(\alpha, \beta)=S M\left(\begin{array}{l}1 \\ p\end{array}\right)$ and $B(\alpha, \beta)=\mathbf{0}_{n \times n}^{p}$. It is clear that $\mathcal{D}(\alpha, \beta)$ lies in $\mathcal{R}^{\prime}(n, p)$. Next let $a(\alpha, \beta) \neq 0$. Then $\beta_{\alpha}$ is non-singular. Suppose that the signature of the matrix $B$ associated to $\beta_{\alpha}$ is $\delta \sigma(\delta= \pm 1)$ as in (C-3) in Section 7. Since $\sigma$ is invariantly defined for $(\alpha, \beta)$, we may write $\sigma(\alpha, \beta)$ for $\sigma$. We define $c(\alpha, \beta)$ and $d(\alpha, \beta)$ by $c(\alpha, \beta)=(n-p+1+\sigma(\alpha, \beta)) / 2$ and $d(\alpha, \beta)=(n-p+1-\sigma(\alpha, \beta)) / 2$ so that $c(\alpha, \beta) \geq d(\alpha, \beta)$. If $c(\alpha, \beta)>d(\alpha, \beta)$, then we can uniquely determine the unit vector $\overline{\mathbf{s}}_{p} \in C_{\alpha}$ in the expression $S \Delta\left(\mathbf{d}_{b(\alpha)}\right) M\left(\begin{array}{l}1 \\ p\end{array}\right)$ so that the index of $B$ is $d(\alpha, \beta)$. If $c(\alpha, \beta)=d(\alpha, \beta)$, then we have no canonical method to determine the orientation of $C_{\alpha}$ in the expression $S \Delta\left(\mathbf{d}_{b(\alpha)}\right) M\left(\begin{array}{l}1 \\ p\end{array}\right)$. There exists a matrix $T \in O(n-p+1)$ such that

$$
{ }^{t} T B T=\Delta(\mathbf{v}(\alpha, \beta), \mathbf{w}(\alpha, \beta)),
$$

where $\mathbf{v}(\alpha, \beta)=\left(v_{1}, \ldots, v_{c(\alpha, \beta)}\right), \mathbf{w}(\alpha, \beta)=\left(w_{1}, \ldots, w_{d(\alpha, \beta)}\right)$ and $v_{1}>\cdots>$ $v_{c(\alpha, \beta)}>0>w_{1}>\cdots>w_{d(\alpha, \beta)}$. When $a(\alpha, \beta) \neq 0$, we define $A(\alpha, \beta)$ and $B(\alpha, \beta)$ by

(8.2) $A(\alpha, \beta)=S \Delta\left(\mathbf{d}_{b(\alpha, \beta)}\right) M\left(\begin{array}{l}1 \\ p\end{array}\right)$,

(8.3) $B(\alpha, \beta)(\mathbf{x}, \mathbf{y})$

$$
=\sqrt{1-b(\alpha, \beta)^{2}}\left\{{ }^{t} \mathbf{x}^{t} M\left(\begin{array}{l}
p \\
n
\end{array}\right)^{t} T\left(E_{c(\alpha, \beta)} \dot{+}\left(-E_{d(\alpha, \beta)}\right)\right) T M\left(\begin{array}{l}
p \\
n
\end{array}\right) \mathbf{y}\right\} \overline{\mathbf{s}}_{p} .
$$


Lemma 8.2. Let $(\alpha, \beta) \in \operatorname{Im}\left(h_{1}\right)$. Then the elements $A(\alpha, \beta)$ and $B(\alpha, \beta)$ are well-defined.

Proof. Suppose that $\alpha=S \Delta\left(\mathbf{d}_{b}\right) M\left(\begin{array}{l}1 \\ p\end{array}\right)=S^{\prime} \Delta\left(\mathbf{d}_{b}\right) M^{\prime}\left(\begin{array}{l}1 \\ p\end{array}\right)$. Let $b(\alpha)=$ 1. Then we have $S M\left(\begin{array}{l}1 \\ p\end{array}\right)=S^{\prime} M^{\prime}\left(\begin{array}{l}1 \\ p\end{array}\right)$. Since $\beta=\mathbf{0}_{n \times n}^{p}$, we have $b(\alpha, \beta)=$ 1. Hence, $A(\alpha, \beta)=S M\left(\begin{array}{l}1 \\ p\end{array}\right)$ and $B(\alpha, \beta)=\mathbf{0}_{n \times n}^{p}$ are well-defined. Let $0 \leq$ $b(\alpha)<1$. Then by Lemma 5.4 there exist matrices $G \in O(p-1)$ and $(\delta) \in$ $O(1)$ such that $S^{\prime}=S\left({ }^{t} G \dot{+}(\delta)\right)$ and $M^{\prime}\left(\begin{array}{l}1 \\ p\end{array}\right)=(G \dot{+}(\delta)) M\left(\begin{array}{l}1 \\ p\end{array}\right)$. Hence, we have $S \Delta\left(\mathbf{d}_{b(\alpha, \beta)}\right) M\left(\begin{array}{l}1 \\ p\end{array}\right)=S^{\prime} \Delta\left(\mathbf{d}_{b(\alpha, \beta)}\right) M^{\prime}\left(\begin{array}{l}1 \\ p\end{array}\right)$. This implies that $A(\alpha, \beta)$ is welldefined by (8.2).

Next we deal with $B(\alpha, \beta)$ in the case $0 \leq b(\alpha)<1$. In the proof we write $c, d, \mathbf{v}$ and $\mathbf{w}$ for $c(\alpha, \beta), d(\alpha, \beta), \mathbf{v}(\alpha, \beta)$ and $\mathbf{w}(\alpha, \beta)$ for simplicity. Suppose that $\alpha=S \Delta\left(\mathbf{d}_{b}\right) M\left(\begin{array}{l}1 \\ p\end{array}\right)=S^{\prime} \Delta\left(\mathbf{d}_{b}\right) M^{\prime}\left(\begin{array}{l}1 \\ p\end{array}\right)$, where $S$ and $S^{\prime}$ are chosen so that if $c>d$, then $\overline{\mathbf{s}}_{p}=\overline{\mathbf{s}}_{p}^{\prime}$. Let $B^{\prime}=\left(b_{i j}^{\prime}\right)$ be the matrix defined by

$$
\beta_{\alpha}\left({ }^{t} \mathbf{m}_{i}^{\prime},{ }^{t} \mathbf{m}_{j}^{\prime}\right)=b_{i j}^{\prime} \overline{\mathbf{s}}_{p}^{\prime}=\left\{\mathbf{m}_{i}^{\prime t} M^{\prime}\left(\begin{array}{l}
p \\
n
\end{array}\right) B^{\prime} M^{\prime}\left(\begin{array}{l}
p \\
n
\end{array}\right)^{t} \mathbf{m}_{j}^{\prime}\right\} \overline{\mathbf{s}}_{p}^{\prime}
$$

and let $B^{\prime}$ be diagonalized as $B^{\prime}={ }^{t} T^{\prime} \Delta(\mathbf{v}, \mathbf{w}) T^{\prime}$ by a matrix $T^{\prime} \in O(n-p+1)$. It is easy to see that

$$
\beta_{\alpha}(\mathbf{x}, \mathbf{y})=\left\{{ }^{t} \mathbf{x}^{t} M\left(\begin{array}{l}
p \\
n
\end{array}\right) B M\left(\begin{array}{l}
p \\
n
\end{array}\right) \mathbf{y}\right\} \overline{\mathbf{s}}_{p}=\left\{{ }^{t} \mathbf{x}^{t} M^{\prime}\left(\begin{array}{l}
p \\
n
\end{array}\right) B^{\prime} M^{\prime}\left(\begin{array}{l}
p \\
n
\end{array}\right) \mathbf{y}\right\} \overline{\mathbf{s}}_{p}^{\prime} .
$$

Hence, if $\overline{\mathbf{s}}_{p}=\delta \overline{\mathbf{s}}_{p}^{\prime}$, then we have

$$
{ }^{t} M\left(\begin{array}{l}
p \\
n
\end{array}\right) B M\left(\begin{array}{l}
p \\
n
\end{array}\right)=\delta^{t} M^{\prime}\left(\begin{array}{l}
p \\
n
\end{array}\right) B^{\prime} M^{\prime}\left(\begin{array}{l}
p \\
n
\end{array}\right) .
$$

Let $a(\alpha, \beta)=0$, and hence $b(\alpha, \beta)=1$. Then $B(\alpha, \beta)$ is well defined since $B(\alpha, \beta)=\mathbf{0}_{n \times n}^{p}$ by $(8.3)$.

Let $a(\alpha, \beta) \neq 0,0 \leq b(\alpha)<1$ and $\sigma(\alpha, \beta)>0$. In this case we have chosen so that $\overline{\mathbf{s}}_{p}^{\prime}=\overline{\mathbf{s}}_{p}$. If $b(\alpha)>0$, we have $\mathbf{m}_{p}^{\prime}=\mathbf{m}_{p}$ and the subspace $\left\{{ }^{t} \mathbf{m}_{p+1}, \ldots,{ }^{t} \mathbf{m}_{n}\right\}$ coincides with $\left\{{ }^{t} \mathbf{m}_{p+1}^{\prime}, \ldots,{ }^{t} \mathbf{m}_{n}^{\prime}\right\}$. If $b(\alpha)=0$, the subspace $\left\{{ }^{t} \mathbf{m}_{p}, \ldots,{ }^{t} \mathbf{m}_{n}\right\}$ coincides with $\left\{{ }^{t} \mathbf{m}_{p}^{\prime}, \ldots,{ }^{t} \mathbf{m}_{n}^{\prime}\right\}$. Whether $b(\alpha)>0$ or $b(\alpha)=$ 0 , we have ${ }^{t} M\left(\begin{array}{l}p \\ n\end{array}\right) B M\left(\begin{array}{l}p \\ n\end{array}\right)={ }^{t} M^{\prime}\left(\begin{array}{l}p \\ n\end{array}\right) B^{\prime} M^{\prime}\left(\begin{array}{l}p \\ n\end{array}\right)$. This gives

$$
{ }^{t} M\left(\begin{array}{l}
p \\
n
\end{array}\right){ }^{t} T \Delta(\mathbf{v}, \mathbf{w}) T M\left(\begin{array}{l}
p \\
n
\end{array}\right)={ }^{t} M^{\prime}\left(\begin{array}{l}
p \\
n
\end{array}\right){ }^{t} T^{\prime} \Delta(\mathbf{v}, \mathbf{w}) T^{\prime} M^{\prime}\left(\begin{array}{l}
p \\
n
\end{array}\right) .
$$

By Lemma 5.5 there exist matrices $T_{1} \in O(c)$ and $T_{2} \in O(d)$ such that $T^{\prime} M^{\prime}\left(\begin{array}{l}p \\ n\end{array}\right)=\left(T_{1}+T_{2}\right) T M\left(\begin{array}{l}p \\ n\end{array}\right)$. Hence we have

$$
{ }^{t} M\left(\begin{array}{l}
p \\
n
\end{array}\right)^{t} T\left(E_{c} \dot{+}\left(-E_{d}\right)\right) T M\left(\begin{array}{l}
p \\
n
\end{array}\right)={ }^{t} M^{\prime}\left(\begin{array}{l}
p \\
n
\end{array}\right)^{t} T^{\prime}\left(E_{c} \dot{+}\left(-E_{d}\right)\right) T^{\prime} M^{\prime}\left(\begin{array}{l}
p \\
n
\end{array}\right) .
$$

Thus $B(\alpha, \beta)$ is well defined by (8.3) in this case. 
Let $a(\alpha, \beta) \neq 0,0 \leq b(\alpha)<1$ and $\sigma(\alpha, \beta)=0$. In this case we need to consider the cases where $\delta$ is 1 or -1 . The proof of the case $\delta=1$ is just the same as above. So let $\delta=-1$. Then we have

$$
\begin{aligned}
& { }^{t} M\left(\begin{array}{l}
p \\
n
\end{array}\right)^{t} T \Delta(\mathbf{v}, \mathbf{w}) T M\left(\begin{array}{l}
p \\
n
\end{array}\right) \\
& \quad={ }^{t} M^{\prime}\left(\begin{array}{l}
p \\
n
\end{array}\right)^{t} T^{\prime} \Delta(-\mathbf{v},-\mathbf{w}) T^{\prime} M^{\prime}\left(\begin{array}{l}
p \\
n
\end{array}\right) \\
& \quad={ }^{t} M^{\prime}\left(\begin{array}{ll}
p \\
n
\end{array}\right)^{t} T^{\prime}\left(\begin{array}{ll}
0 & E_{c} \\
E_{c} & 0
\end{array}\right) \Delta(-\mathbf{w},-\mathbf{v})\left(\begin{array}{ll}
0 & E_{c} \\
E_{c} & 0
\end{array}\right) T^{\prime} M^{\prime}\left(\begin{array}{l}
p \\
n
\end{array}\right) .
\end{aligned}
$$

By Lemmas 5.2 and 5.5 we have $\mathbf{v}=-\mathbf{w}$ and there exists $T_{1}, T_{2} \in O(c)$ such that $T^{\prime} M^{\prime}\left(\begin{array}{l}p \\ n\end{array}\right)=\left(\begin{array}{cc}0 & E_{c} \\ E_{c} & 0\end{array}\right)\left(T_{1}+T_{2}\right) T M\left(\begin{array}{l}p \\ n\end{array}\right)$. Hence, we have

$$
\begin{aligned}
& \left\{{ }^{t} \mathbf{x}^{t} M^{\prime}\left(\begin{array}{l}
p \\
n
\end{array}\right)^{t} T^{\prime}\left(E_{c} \dot{+}\left(-E_{c}\right)\right) T^{\prime} M^{\prime}\left(\begin{array}{l}
p \\
n
\end{array}\right) \mathbf{y}\right\} \overline{\mathbf{s}}_{p}^{\prime}
\end{aligned}
$$

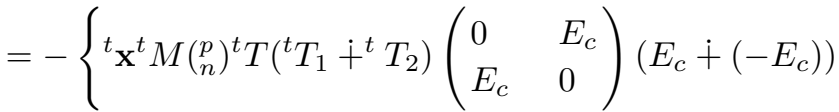

$$
\begin{aligned}
& \left.\times\left(\begin{array}{ll}
0 & E_{c} \\
E_{c} & 0
\end{array}\right)\left(T_{1}+T_{2}\right) T M\left(\begin{array}{l}
p \\
n
\end{array}\right) \mathbf{y}\right\} \overline{\mathbf{s}}_{p}^{\prime} \\
& =\left\{{ }^{t} \mathbf{x}^{t} M\left(\begin{array}{l}
p \\
n
\end{array}\right)^{t} T\left(E_{c} \dot{+}\left(-E_{c}\right)\right) T M\left(\begin{array}{l}
p \\
n
\end{array}\right) \mathbf{y}\right\} \overline{\mathbf{s}}_{p} .
\end{aligned}
$$

Thus $B(\alpha, \beta)$ is well defined by (8.3).

We here state the properties of $\mathcal{D}(\alpha, \beta)$, which are easily proved from Remark 2.4.

Proposition 8.3. Let $(\alpha, \beta) \in \operatorname{Im}\left(h_{1}\right)$. Then we have the following properties.

(1) If $(\alpha, \beta) \in \mathcal{K}^{\prime}(n, p)$, then $\mathcal{D}(\alpha, \beta)=(\alpha, \beta)$.

(2) The image of $\mathcal{D}$ coincides with $\mathcal{K}^{\prime}(n, p)$.

(3) If $a(\alpha, \beta)=0$, then $\mathcal{D}(\alpha, \beta) \in \mathcal{R}^{\prime}(n, p)$.

(4) If $\alpha \in \Sigma K^{\prime}(n, p),(\alpha, \beta) \in \Sigma^{n-p+1,0}(n, p)$ with $\sigma(\alpha, \beta)$, then $\mathcal{D}(\alpha, \beta) \in$ $\Sigma \mathcal{K}^{\prime}(n, p, \sigma(\alpha, \beta))$.

(5) If $a(\alpha, \beta) \neq 0$ and $0<b(\alpha)<1$, then we have $0<b(\alpha, \beta)<1$.

Let $G_{\ell, m-\ell}$ be the grassman manifold of $\ell$-dimensional subspaces of $\mathbf{R}^{m}$. An element of $G_{\ell, m-\ell}$ is expressed by an $\ell$-dimensional subspace $V$ of $\mathbf{R}^{m}$. The proof of the following lemma is left to the reader. 
Lemma 8.4. Let $\left\{\alpha^{k}\right\}$ be a sequence which converges to $\alpha$ in $K^{\prime}(n, p)$. Assume that if $0<b(\alpha)<1$, then $0<b\left(\alpha^{k}\right)<1$ for all $k$. Then we have the followings.

(1) The sequence $\left\{C_{\alpha^{k}}\right\}$ converges to $C_{\alpha}$ in $R P^{p-1}$.

(2) If $0<b(\alpha)<1$, then the sequence $\left\{\operatorname{Ker}\left(\alpha^{k}\right)\right\}$ converges to $\operatorname{Ker}(\alpha)$ in $G_{n-p, p}$.

(3) The sequence $\left\{K_{\alpha^{k}}\right\}$ converges to $K_{\alpha}$ in $G_{n-p+1, p-1}$.

Corollary 8.5. Let $\left\{\alpha^{k}\right\}$ be a sequence which converges to $\alpha$ in $K^{\prime}(n, p)$ such that $0<b(\alpha)<1$, and $0<b\left(\alpha^{k}\right)<1$ for all $k$. Let $\mathbf{m}$ be a unit vector of $K_{\alpha}$ with $\mathbf{m} \perp \operatorname{Ker}(\alpha)$. Then for sufficiently large $k$ there exists a unit vector $\mathbf{m}^{k}$ of $K_{\alpha^{k}}$ with $\mathbf{m}^{k} \perp \operatorname{Ker}\left(\alpha^{k}\right)$ such that $\lim _{k \rightarrow \infty} \mathbf{m}^{k}=\mathbf{m}$.

Proposition 8.6. The map $\mathcal{D}=(A, B): \operatorname{Im}\left(h_{1}\right) \rightarrow \mathcal{K}^{\prime}(n, p)$ is continuous.

Proof. Let $\left\{\left(\alpha^{k}, \beta^{k}\right)\right\}$ be a sequence which converges to $(\alpha, \beta)$ in $\operatorname{Im}\left(h_{1}\right)$ with diagonalizations

$$
\alpha^{k}=S^{k} \Delta\left(\mathbf{d}_{b\left(\alpha^{k}\right)}\right) M^{k}\left(\begin{array}{l}
1 \\
p
\end{array}\right) \quad \text { and } \quad \alpha=S \Delta\left(\mathbf{d}_{b(\alpha)}\right) M\left(\begin{array}{l}
1 \\
p
\end{array}\right) .
$$

Since $\lim _{k \rightarrow \infty}{ }^{t} S \alpha_{k}{ }^{t} M={ }^{t} S \alpha^{t} M$, we have

$$
\begin{aligned}
\lim _{k \rightarrow \infty}{ }^{t} S S^{k} \Delta\left(\mathbf{d}_{b\left(\alpha^{k}\right)}\right) M\left(\begin{array}{l}
1 \\
p
\end{array}\right)^{t} M & =\Delta\left(\mathbf{d}_{b(\alpha)}\right)\left(M\left(\begin{array}{l}
1 \\
p
\end{array}\right)^{t} M\left(\begin{array}{l}
1 \\
p
\end{array}\right), M\left(\begin{array}{l}
1 \\
p
\end{array}\right)^{t} M\left(\begin{array}{l}
p+1 \\
n
\end{array}\right)\right) \\
& =\Delta\left(\mathbf{d}_{b(\alpha)}\right)\left(E_{p}, \mathbf{0}_{p \times(n-p)}\right) .
\end{aligned}
$$

By Lemma 5.6 we have matrices $\delta\left({ }^{t} S S^{k}\right)$ which, if $b(\alpha)<1$, is written as $G^{k} \dot{+}(x)$ such that $\lim _{k \rightarrow \infty}\left({ }^{t} S S^{k}-\delta\left({ }^{t} S S^{k}\right)\right)=\mathbf{0}_{p \times p}$. Furthermore, if $0<$ $b(\alpha)<1$, then $\lim _{k \rightarrow \infty} \delta\left({ }^{t} S S^{k}\right) M^{k}\left(\begin{array}{l}1 \\ p\end{array}\right)^{t} M=\left(E_{p}, \mathbf{0}_{p \times(n-p)}\right)$, and if $b(\alpha)=0$, then $\lim _{k \rightarrow \infty} G^{k} M^{k}\left(\begin{array}{c}1 \\ p-1\end{array}\right)^{t} M=\left(E_{p-1}, \mathbf{0}_{(p-1) \times(n-p+1)}\right)$.

Case (i): Suppose $a(\alpha, \beta)=0$.

We note that $b(\alpha) \neq 0$. Since the set of eigen values of a matrix is continuous with respect to components of matrices, we have $\lim _{k \rightarrow \infty} a\left(\alpha^{k}, \beta^{k}\right)=$ $a(\alpha, \beta)=0$. By $(8.1)$ we have

$$
\lim _{k \rightarrow \infty} b\left(\alpha^{k}, \beta^{k}\right)=\lim _{k \rightarrow \infty} \frac{b\left(\alpha^{k}\right)}{\sqrt{a\left(\alpha^{k}, \beta^{k}\right)^{2}+b\left(\alpha^{k}\right)^{2}}}=1 .
$$


Hence, we have

$$
\begin{aligned}
\lim _{k \rightarrow \infty}{ }^{t} S A\left(\alpha^{k}, \beta^{k}\right)^{t} M & =\lim _{k \rightarrow \infty}{ }^{t} S S^{k} \Delta\left(\mathbf{d}_{b\left(\alpha^{k}, \beta^{k}\right)}\right) M^{k}\left(\begin{array}{l}
1 \\
p
\end{array}\right)^{t} M \\
& =\lim _{k \rightarrow \infty}{ }^{t} S\left(S^{k} M^{k}\left(\begin{array}{l}
1 \\
p
\end{array}\right)+S^{k}\left(\Delta\left(\mathbf{d}_{b\left(\alpha^{k}, \beta^{k}\right)}-E_{p}\right) M^{k}\left(\begin{array}{l}
1 \\
p
\end{array}\right)\right)^{t} M\right. \\
& =\lim _{k \rightarrow \infty}{ }^{t} S S^{k} M^{k}\left(\begin{array}{l}
1 \\
p
\end{array}\right)^{t} M \\
& \left.=\lim _{k \rightarrow \infty}{ }^{t} \delta\left({ }^{t} S S^{k}\right)\right) M^{k}\left(\begin{array}{l}
1 \\
p
\end{array}\right)^{t} M \\
& =\lim _{k \rightarrow \infty}\left(E_{p}, \mathbf{0}_{p \times(n-p)}\right) \\
& ={ }^{t} S A(\alpha, \beta)^{t} M .
\end{aligned}
$$

Since $\lim _{k \rightarrow \infty} b\left(\alpha^{k}, \beta^{k}\right)=1$ and the norm $\left\|\beta_{\alpha^{k}}^{k}\right\|$ converges to 0 , it follows that $\lim _{k \rightarrow \infty} B\left(\alpha^{k}, \beta^{k}\right)=\mathbf{0}$. Therefore, if $a(\alpha, \beta)=0$, then $\mathcal{D}$ is continuous at $(\alpha, \beta)$.

Case (ii): Suppose $a(\alpha, \beta) \neq 0$.

Since we are working in $\operatorname{Im}\left(h_{1}\right)$, this yields $0 \leq b(\alpha)<1$. Then we have

$$
\begin{aligned}
\lim _{k \rightarrow \infty}{ }^{t} S A\left(\alpha^{k}, \beta^{k}\right)^{t} M & =\lim _{k \rightarrow \infty}{ }^{t} S S^{k} \Delta\left(\mathbf{d}_{b\left(\alpha^{k}, \beta^{k}\right)}\right) M^{k}\left(\begin{array}{l}
1 \\
p
\end{array}\right)^{t} M \\
& =\lim _{k \rightarrow \infty} \delta\left({ }^{t} S S^{k}\right) \Delta\left(\mathbf{d}_{b\left(\alpha^{k}, \beta^{k}\right)}\right) M^{k}\left(\begin{array}{l}
1 \\
p
\end{array}\right)^{t} M \\
& =\lim _{k \rightarrow \infty} \Delta\left(\mathbf{d}_{b\left(\alpha^{k}, \beta^{k}\right)}\right) \delta\left({ }^{t} S S^{k}\right) M^{k}\left(\begin{array}{l}
1 \\
p
\end{array}\right)^{t} M \\
& =\lim _{k \rightarrow \infty} \Delta\left(\mathbf{d}_{b\left(\alpha^{k}, \beta^{k}\right)}\right)\left(E_{p}, \mathbf{0}_{p \times(n-p)}\right) \\
& ={ }^{t} S A(\alpha, \beta)^{t} M .
\end{aligned}
$$

Thus we have proved $\lim _{k \rightarrow \infty} A\left(\alpha^{k}, \beta^{k}\right)=A(\alpha, \beta)$.

We prove the continuity of $B(\alpha, \beta)$. We note that if $\sigma(\alpha, \beta)>0$, then we have chosen a unit basis $\overline{\mathbf{s}}_{p}$ so that the index of $B$ is less than $(n-p+1) / 2$ and that if $\sigma(\alpha, \beta)=0$, then we chose $\overline{\mathbf{s}}_{p}$ arbitrarily. For a sufficiently large number $k$ we set $\overline{\mathbf{s}}_{p}^{k}=\operatorname{pr}\left(C_{\alpha^{k}}\right)\left(\overline{\mathbf{s}}_{p}\right) /\left\|\operatorname{pr}\left(C_{\alpha^{k}}\right)\left(\overline{\mathbf{s}}_{p}\right)\right\|$. If $0<b(\alpha)<1$, then it follows from Corollary 8.5 that for the vector ${ }^{t} \mathbf{m}_{p}$, there exists a unit vector ${ }^{t} \mathbf{m}_{p}^{k}$ for a sufficiently large number $k$ with ${ }^{t} \mathbf{m}_{p}^{k} \in K_{\alpha^{k}}$ and ${ }^{t} \mathbf{m}_{p}^{k} \perp \operatorname{Ker}\left(\alpha^{k}\right)$ such that $\lim _{k \rightarrow \infty}{ }^{t} \mathbf{m}_{p}^{k}={ }^{t} \mathbf{m}_{p}$. For the orthonormal basis ${ }^{t} \mathbf{m}_{p}, \ldots,{ }^{t} \mathbf{m}_{n}$ of $K_{\alpha}$, we set $\mathbf{a}_{j}^{k}=\operatorname{pr}\left(K_{\alpha^{k}}\right)\left({ }^{t} \mathbf{m}_{j}\right)(j=p+1, \ldots, n)$. There is a large number $k_{0}$ such that if $k>k_{0}$, then ${ }^{t} \mathbf{m}_{p}^{k},{ }^{t} \mathbf{a}_{p+1}^{k}, \ldots,{ }^{t} \mathbf{a}_{n}^{k}$ are linearly independent. By applying the Gram-Schmidt orthonormalization process to ${ }^{t} \mathbf{m}_{p}^{k},{ }^{t} \mathbf{a}_{p+1}^{k}, \ldots,{ }^{t} \mathbf{a}_{n}^{k}$ putted in this order, we obtain an orthonormal basis, say ${ }^{t} \mathbf{m}_{p}^{k}, \ldots,{ }^{t} \mathbf{m}_{n}^{k}$. It is easily verified 
that $\lim _{k \rightarrow \infty}{ }^{t} \mathbf{m}_{j}^{k}={ }^{t} \mathbf{m}_{j}$ for $j=p, \ldots, n$. If $b(\alpha)=0$, then there exists an orthonormal basis ${ }^{t} \mathbf{m}_{p}, \ldots,{ }^{t} \mathbf{m}_{n}$ of $K_{\alpha}=\operatorname{Ker}(\alpha)$. We set $\mathbf{a}_{j}^{k}=\operatorname{pr}\left(K_{\alpha^{k}}\right)\left({ }^{t} \mathbf{m}_{j}\right)$ $(j=p, \ldots, n)$. By the similar arguments we obtain an orthonormal basis, say ${ }^{t} \mathbf{m}_{p}^{k}, \ldots,{ }^{t} \mathbf{m}_{n}^{k}$ such that $\lim _{k \rightarrow \infty}{ }^{t} \mathbf{m}_{j}^{k}={ }^{t} \mathbf{m}_{j}$ for $j=p, \ldots, n$. Suppose that $S^{k}, S \in O(p)$ and $M^{k}, M \in O(n)$ in the expressions (8.2) and (8.3) are chosen to have these column and row vectors.

For $\left(\alpha^{k}, \beta^{k}\right)$ we define the matrix $B^{k}$ by $\beta_{\alpha^{k}}^{k}\left({ }^{t} \mathbf{m}_{i}^{k},{ }^{t} \mathbf{m}_{j}^{k}\right)=b_{i j}^{k} \overline{\mathbf{s}}_{p}^{k}$, namely

$$
\beta_{\alpha^{k}}^{k}(\mathbf{x}, \mathbf{y})=\left\{{ }^{t} \mathbf{x}^{t} M^{k}\left(\begin{array}{l}
p \\
n
\end{array}\right) B^{k} M^{k}\left(\begin{array}{l}
p \\
n
\end{array}\right) \mathbf{y}\right\} \overline{\mathbf{s}}_{p}^{k} .
$$

Then we have

$$
\begin{aligned}
b_{i j} \overline{\mathbf{s}}_{p} & =\operatorname{pr}\left(C_{\alpha}\right) \circ \beta\left({ }^{t} \mathbf{m}_{i},{ }^{t} \mathbf{m}_{j}\right) \\
& =\lim _{k \rightarrow \infty} \operatorname{pr}\left(C_{\alpha^{k}}\right) \circ \beta^{k}\left({ }^{t} \mathbf{m}_{i}^{k},{ }^{t} \mathbf{m}_{j}^{k}\right) \\
& =\lim _{k \rightarrow \infty} \beta_{\alpha^{k}}^{k}\left({ }^{t} \mathbf{m}_{i}^{k},{ }^{t} \mathbf{m}_{j}^{k}\right) \\
& =\lim _{k \rightarrow \infty} b_{i j}^{k} \overline{\mathbf{s}}_{p}^{k} \\
& =\left(\lim _{k \rightarrow \infty} b_{i j}^{k}\right) \overline{\mathbf{s}}_{p} .
\end{aligned}
$$

Hence, we have $\lim _{k \rightarrow \infty} B^{k}=B$.

Since $a(\alpha, \beta) \neq 0, \beta_{\alpha}$ is non-singular. By the choice of $\overline{\mathbf{s}}_{p}$, we have $c(\alpha, \beta) \geq d(\alpha, \beta)$. Therefore, we can assert that if $k$ is sufficiently large, then $\beta_{\alpha^{k}}^{k}$ is non-singular, and $c\left(\alpha^{k}, \beta^{k}\right)=c(\alpha, \beta), d\left(\alpha^{k}, \beta^{k}\right)=d(\alpha, \beta)$ and $\sigma\left(\alpha^{k}, \beta^{k}\right)=\sigma(\alpha, \beta)$. Suppose that $B^{k}$ is diagonalized, by a matrix $T^{k}$, as $T^{k} B^{k}\left({ }^{t} T^{k}\right)=\Delta(\mathbf{v}, \mathbf{w})$ with $v_{1}^{k} \geq \cdots \geq v_{c}^{k}>0>w_{1}^{k} \geq \cdots \geq w_{d}^{k}$ for large $k$. Since $\lim _{k \rightarrow \infty} B^{k}=B$, we have $\lim _{k \rightarrow \infty}{ }^{t} T^{k} \Delta(\mathbf{v}, \mathbf{w}) T^{k}={ }^{t} T \Delta(\mathbf{v}, \mathbf{w}) T$. Hence,

$$
\lim _{k \rightarrow \infty} T\left({ }^{t} T^{k}\right) \Delta(\mathbf{v}, \mathbf{w}) T^{k}\left({ }^{t} T\right)=\Delta(\mathbf{v}, \mathbf{w})
$$

Then we have matrices $\delta\left(T\left({ }^{t} T^{k}\right)\right)$ described in Lemma 5.3. Thus, we have

$$
\begin{aligned}
\lim _{k \rightarrow \infty} T\left({ }^{t} T^{k}\right)\left(E_{c} \dot{+}\left(-E_{d}\right)\right) T^{k}\left({ }^{t} T\right) & =\lim _{k \rightarrow \infty} \delta\left(T\left({ }^{t} T^{k}\right)\right)\left(E_{c} \dot{+}\left(-E_{d}\right)\right)^{t} \delta\left(T\left({ }^{t} T^{k}\right)\right) \\
& =\lim _{k \rightarrow \infty}\left(E_{c} \dot{+}\left(-E_{d}\right)\right) \delta\left(T\left({ }^{t} T^{k}\right)\right)^{t} \delta\left(T\left({ }^{t} T^{k}\right)\right) \\
& =\left(E_{c} \dot{+}\left(-E_{d}\right)\right) .
\end{aligned}
$$

Therefore, we have $\lim _{k \rightarrow \infty}{ }^{t} T^{k}\left(E_{c\left(\alpha^{k}, \beta^{k}\right)} \dot{+}\left(-E_{d\left(\alpha^{k}, \beta^{k}\right)}\right)\right) T^{k}={ }^{t} T\left(E_{c} \dot{+}\left(-E_{d}\right)\right)$ $T$. Since $\lim _{k \rightarrow \infty}{ }^{t} \mathbf{m}_{j}^{k}={ }^{t} \mathbf{m}_{j}$ for $j=p, \ldots, n$, we have

$$
\lim _{k \rightarrow \infty}{ }^{t} M^{k}\left(\begin{array}{l}
p \\
n
\end{array}\right)^{t} T^{k}\left(E_{c} \dot{+}\left(-E_{d}\right)\right) T^{k} M^{k}\left(\begin{array}{l}
p \\
n
\end{array}\right)={ }^{t} M\left(\begin{array}{l}
p \\
n
\end{array}\right)^{t} T\left(E_{c} \dot{+}\left(-E_{d}\right)\right) T M\left(\begin{array}{l}
p \\
n
\end{array}\right) .
$$


For $\mathbf{x}, \mathbf{y} \in \mathbf{R}^{n}$, set $\mathbf{x}^{k}=\operatorname{pr}\left(K_{\alpha^{k}}\right)(\mathbf{x}), \mathbf{y}^{k}=\operatorname{pr}\left(K_{\alpha^{k}}\right)(\mathbf{y}), \mathbf{x}^{0}=\operatorname{pr}\left(K_{\alpha}\right)(\mathbf{x})$ and $\mathbf{y}^{0}=\operatorname{pr}\left(K_{\alpha}\right)(\mathbf{y})$. By the definition (8.3) we have

$$
\begin{aligned}
B(\alpha, \beta) & (\mathbf{x}, \mathbf{y}) \\
& =B(\alpha, \beta)\left(\mathbf{x}^{0}, \mathbf{y}^{0}\right) \\
& =\sqrt{1-b(\alpha, \beta)^{2}}\left\{\left({ }^{t} \mathbf{x}^{0}\right)^{t} M\left(\begin{array}{l}
p \\
n
\end{array}\right)^{t} T\left(E_{c}+\left(-E_{d}\right)\right) T M\left(\begin{array}{l}
p \\
n
\end{array}\right) \mathbf{y}^{0}\right\} \overline{\mathbf{s}}_{p} \\
& =\lim _{k \rightarrow \infty} \sqrt{1-b\left(\alpha^{k}, \beta^{k}\right)^{2}}\left\{\left({ }^{t} \mathbf{x}^{k}\right)^{t} M^{k}\left(\begin{array}{l}
p \\
n
\end{array}\right)^{t} T^{k}\left(E_{c}+\left(-E_{d}\right)\right) T^{k} M^{k}\left(\begin{array}{l}
p \\
n
\end{array}\right) \mathbf{y}^{k}\right\} \overline{\mathbf{s}}_{p}^{k} \\
& =\lim _{k \rightarrow \infty} B\left(\alpha^{k}, \beta^{k}\right)\left(\mathbf{x}^{k}, \mathbf{y}^{k}\right) \\
& =\lim _{k \rightarrow \infty} B\left(\alpha^{k}, \beta^{k}\right)(\mathbf{x}, \mathbf{y}) .
\end{aligned}
$$

This shows $\lim _{k \rightarrow \infty} B\left(\alpha^{k}, \beta^{k}\right)=B(\alpha, \beta)$. Therefore, $B(\alpha, \beta)$ is continuous at a point $(\alpha, \beta)$ with $a(\alpha, \beta) \neq 0$.

This completes the proof.

Proof of Proposition 8.1. We define a deformation retraction $\mathcal{D}_{\lambda}$ of $\operatorname{Im}\left(h_{1}\right)$ to $\mathcal{K}^{\prime}(n, p)$ by

$$
\mathcal{D}_{\lambda}(\alpha, \beta)=(1-\lambda)(\alpha, \beta)+\lambda \mathcal{D}(\alpha, \beta)=\left(A_{\lambda}(\alpha, \beta), B_{\lambda}(\alpha, \beta)\right),
$$

where

$$
\begin{gathered}
A_{\lambda}(\alpha, \beta)=(1-\lambda) \alpha+\lambda A(\alpha, \beta)=S \Delta\left(\mathbf{d}_{(1-\lambda) b(\alpha)+\lambda b(\alpha, \beta)}\right) M\left(\begin{array}{l}
1 \\
p
\end{array}\right), \\
B_{\lambda}(\alpha, \beta)=(1-\lambda) \beta+\lambda B(\alpha, \beta) .
\end{gathered}
$$

By Propositin 8.6, $\mathcal{D}_{\lambda}(\alpha, \beta)$ is continuous with respect to $\alpha, \beta$ and $\lambda$. We first prove that $\mathcal{D}_{\lambda}$ is a map into $\operatorname{Im}\left(h_{1}\right)$. In fact, if $b(\alpha)=1$ and $\beta=\mathbf{0}_{n \times n}^{p}$, then $\mathcal{D}(\alpha, \beta)=(\alpha, \beta)$, and hence $\mathcal{D}_{\lambda}(\alpha, \beta)=(\alpha, \beta)=\left(\alpha, \mathbf{0}_{n \times n}^{p}\right)$ by Proposition $8.3(1)$.

If $b(\alpha)=0$, then $b(\alpha, \beta)=0$, and hence $(1-\lambda) b(\alpha)+\lambda b(\alpha, \beta)=0$. This implies that $A_{\lambda}(\alpha, \beta)$ is always equal to $\alpha$ for such $(\alpha, \beta)$. We have that if $b(\alpha)=0$, then $B_{\lambda}(\alpha, \beta)$ is non-singular, since $(1-\lambda) \Delta(\mathbf{v}(\alpha, \beta), \mathbf{w}(\alpha, \beta))+$ $\lambda \sqrt{1-b(\alpha, \beta)^{2}}\left(E_{c}+\left(-E_{d}\right)\right)$ is non-singular. This shows that $\mathcal{D}_{\lambda}(\alpha, \beta)$ lies in $\operatorname{Im}\left(h_{1}\right)$. If $0<b(\alpha) \leq 1$, then we have $0<(1-\lambda) b(\alpha)+\lambda b(\alpha, \beta) \leq 1$.

We have that $\mathcal{D}_{0}=i d_{\operatorname{Im}\left(h_{1}\right)}$ by definition, $\operatorname{Im} \mathcal{D}_{1}=\mathcal{K}^{\prime}(n, p)$ and $\mathcal{D}_{\lambda} \mid \mathcal{K}^{\prime}(n, p)$ $=i d_{\mathcal{K}^{\prime}(n, p)}$ by Proposition 8.3 (1) and (3). This completes the proof. 


\section{References}

[An1] Ando, Y., On the elimination of Morin singularities, J. Math. Soc. Japan, 37 (1985), 471-487.

[An2] - Folding maps and the surgery theory on manifolds, J. Math. Soc. Japan, 53 (2001), 357-382.

[An3] $\longrightarrow$ Fold-maps and the space of base point preserving maps of spheres, J. Math. Kyoto. Univ., 41 (2002), 691-735.

[An4] - Existence theorems of fold-maps, submitted.

[An5] - An invariant of maps with prescribed singularities, in preparation.

[At] Atiyah, M. F., Thom complexes, Proc. London Math. Soc., 11 (1961), 291-310.

[Bo] Boardman, J. M., Singularities of differentiable maps, IHES Publ. Math., 33 (1967), 21-57.

[B-R] Burlet, O. and de Rham, G., Sur certaines applications génériques d'une variété close à 3 dimensions dans le plan, Enseign. Math., 20 (1974), 275-292.

[E1] Èliašberg, J. M., On singularities of folding type, Math. USSR Izv., 4 (1970), 11191134.

[E2] - Surgery of singularities of smooth mappings, Math. USSR Izv., 6 (1972), 1302-1326.

[G1] Gromov, M., Stable mappings of foliations into manifolds, Math. USSR Izv., 3 (1969), 671-694.

[G2] - Partial Differential Relations, Springer-Verlag, 1986.

[H] Hirzebruch, F., Topological Methods in Algebraic Geometry, Springer-Verlag, 1956.

[K-N] Kobayashi, S. and Nomizu, K., Foundations of Differential Geometry, Vol.1, Interscience Publishers, 1963.

[L] Levine, H. I., Singularities of differentiable maps, Proc. Liverpool Singularities Symposium, Springer Lecture Notes in Math., 192 (1971), 1-85.

[M-M] Madsen, I. and Milgram, R. J., The Classifying Spaces for Surgery and Cobordism of Manifolds, Princeton, 1979.

[Ma] Mather, J., Stability of $C^{\infty}$ mappings: VI. The nice dimensions, Proceedings of Liverpool Singularities, Lecture Notes in Math., 192 (1971), 207-253.

[M-S] Milnor, J. and Spanier, E., Two remarks on fiber homotopy type, Pacific J. Math., 10 (1960), 585-590.

[Mo] Morin, B., Formes canoniques des singularités d'une application différentiable, C. R. Acad. Sci. Paris, 260 (1965), 5662-5665; 6503-6506.

[Sa] Saeki, O., Notes on the topology of folds, J. Math. Soc. Japan, 44 (1992), 551-566.

[S-S] Saeki, O. and Sakuma, K., Special generic maps of 4-manifolds and compact complex analytic surfaces, Math. Ann., 313 (1999), 617-633.

[Spa1] Spanier, E., Infinite symmetric products, function spaces, and duality, Ann. of Math., 69 (1959), 142-198.

[Spa2] — Function spaces and duality, Ann. of Math., 70 (1959), 338-378.

[T] Thom, R., Les singularités des applications différentiables, Ann. Inst. Fourier, 6 (1955-56), 43-87.

[Tsu] Tsuchiya, A., Characteristic classes for spherical fiber spaces, Nagoya Math. J., 43 (1971), 1-39.

[W] Whitney, H., Complex Analytic Varieties, Addison-Wesley, 1972. 